\title{
Study of the stellar line-strength indices and kinematics along bars ${ }^{\star}, \star \star$
}

\section{Bar age and metallicity gradients}

\author{
I. Pérez ${ }^{1,2,3, \star \star \star, \dagger}$, P. Sánchez-Blázquez ${ }^{4, \ddagger}$, and A. Zurita ${ }^{2,3, \S}$ \\ 1 Kapteyn Astronomical Institute, University of Groningen, Postbus 800, Groningen 9700 AV, The Netherlands \\ e-mail: isa@astro.rug.nl \\ 2 Departamento de Física teórica y del Cosmos, Campus de Fuentenueva, Universidad de Granada, 18071 Granada, Spain \\ 3 Instituto Carlos I de Física Teórica y Computación, Spain \\ e-mail: azurita@ugr.es \\ 4 Jeremiah Horrocks Institute for Astrophysics \& Supercomputing, University of Central Lancashire, PR1 2HE Preston, UK \\ e-mail: psanchez-blazquez@uclan.ac.uk
}

Accepted 3 october 2008 / Received 8 December 2008

\section{ABSTRACT}

\begin{abstract}
Aims. This is the first paper of a series that aims to understand the formation and evolution of bars in early-type spirals and their influence in the evolution of the galaxy.

Methods. Optical long-slit spectra along the bar major-axis of a sample of 20 galaxies are analyzed. Velocity and velocity dispersion profiles along the bar are presented. Line-strength indices in the bar region are also measured to derive stellar mean-age and metallicity distributions along the bars using stellar population models.

Results. We obtain mean ages, metallicities and chemical abundances along the bar of 20 galaxies with morphological types from SB0 to SBbc. The main result is that we find a large variation in age and metallicity along the bar in $45 \%$ of our sample. We find three different types of bars according to their metallicity and age distribution along the radius: 1) Bars with negative metallicity gradients. They show a mean young/intermediate population $(<2 \mathrm{Gyr})$, and have amongst the lowest stellar maximum central velocity dispersion of the sample; 2) bars with null metallicity gradients. These galaxies do not show any gradient in their metallicity distribution along the bar and have negative age gradients (i.e. younger populations at the bar end); 3) bars with positive metallicity gradients, i.e. more metal rich at the bar ends. These galaxies are predominantly those with higher velocity dispersion and an older mean population. We found no significant correlation between the age and metallicity distribution, and bar/galaxy parameters such as the AGN presence, size or the bar strength. From the kinematics, we find that all the galaxies show a disk-like central component.

Conclusions. The results from the metallicity and age gradients indicate that most galaxies with high central stellar velocity dispersion host bars that could have been formed more than 3 Gyr ago, while galaxies with lower central velocity dispersions show a wider distribution in their population and age gradients. A few bars show characteristics compatible with having been formed less than $<2$ Gyr ago. However, we do not have a definite answer to explain the observed gradients and these results place strong constrains on models of bar formation and evolution. The distribution of mean stellar population parameters in the bar with respect to $\sigma$ is similar to that found in bulges, indicating a close link in the evolution of both components. The disk-like central components also show the important role played by bars in the secular evolution of the central structure.
\end{abstract}

Key words. galaxies: spiral - galaxies: abundances - galaxies: evolution - galaxies: formation - galaxies: structure galaxies: kinematics and dynamics

\section{Introduction}

Bars are known to be efficient mechanisms to redistribute angular momentum and matter in galaxies up to large distances and, therefore, they are expected to play an important role in the secular evolution of disk galaxies. The bar gravity torques make the gas lose angular momentum which, in turn, provokes an inflow of gas towards the central parts. This inflow of gas

* Based on observations obtained at Siding Spring Observatory (RSAA, ANU, Australia) and the INT telescope at the ING, La Palma, Spain.

$\star \star$ Appendices are only available in electronic form at

http://www . aanda.org

$\star \star \star$ Veni Fellow.

Associate Researcher.

Marie Curie Fellow.

$\S$ Retorno J.A. Fellow. accumulates in central mass concentrations (CMC), fueling the central black hole (e.g. Shlosman et al. 1989) and, possibly, forming a stellar bulge. Bars are found in $\sim 60 \%$ of spiral galaxies in the local Universe (e.g. Knapen et al. 2000; Eskridge et al. 2000; Whyte et al. 2002; Menéndez-Delmestre et al. 2007; Marinova \& Jogee 2007), or 30\% if only strong bars are considered. Despite their obvious importance in disk galaxy evolution, their own fate is still a matter of debate. Several mechanisms are able to destroy the bar: for example; 1) the bar may dissolve in the presence of a sufficiently massive central component (e.g. a black hole, Friedli \& Pfenniger 1991; Friedli \& Benz 1993; Norman et al. 1996; Berentzen et al. 1998; Fukuda et al. 2000; Bornaud \& Combes 2002; Shen \& Sellwood 2004; Athanassoula et al. 2005; Bornaud et al. 2005). However, the masses of the CMC needed to destroy the bar are much larger than those of the most super massive black holes found so far in disk 
galaxies (Shen \& Selwood 2004; Athanassoula et al. 2005; Ferrarese \& Ford 2005). 2) Transfer of angular momentum from infalling gas to the bar has also been proposed as a mechanism that could strongly weaken the bar. The combined effect of this angular momentum transfer and the CMC can destroy the bar in Sb-Sc galaxies in 1-2 Gyr (Bournaud \& Combes 2002). Then, if gas is present, a new bar could form with characteristics different from those of the parent bar. However, simulations of the effect of gas on the stellar evolution of a bar embedded in a live halo (Berentzen et al. 2007) have shown that, although there are some structural differences in bars evolving with and without gas, in both cases the stellar bar can survive at least 5 Gyr (the total computation time of these simulations).

Do all bars go through these processes? Do some bars die while others are robust over many Hubble times? Previous studies have tried to put some limits on the age of the bars by analyzing the evolution with redshift of the bar's fraction in galaxies. These studies have also obtained discrepant results: Some authors have found a fraction of barred galaxies at $z>0.5$ lower than the local fraction (Abraham et al. 1999), although some authors claim that this may be the consequence of selection effects, due to the high angular resolution needed to find bars (Elmegreen et al. 2004; although see van den Bergh et al. 2002). In order to avoid the problem of angular resolution, several studies have carried out this analysis using the ACS camera on the HST: Elmegreen et al. (2004) and Jogee et al. (2004) found the same bar fraction $(\sim 0.4)$ at redshift $z=1.1$ as in the local Universe, suggesting that the bar dissolution cannot be common during a Hubble time unless of course the bar formation rate is comparable to the bar destruction rate. On the contrary, Sheth et al. (2008), using images from the COSMOS survey (Scoville et al. 2007) and using a larger sample than previous studies, found that the bar fraction at $z=0.84$ is one-third of the present-day value. They also found a much stronger evolution for low mass galaxies and late-type spectral types. Part of the discrepant results may be due to the selection effects and other systematic effects that still need to be further investigated.

Detailed analysis of the stellar populations in the bar region of local galaxies can shed some light on the formation and evolution of bars. Gadotti \& de Souza (2006) obtained the color and color gradients in the bar region of a sample of 18 barred galaxies. They interpreted the color differences as differences in stellar ages. They concluded that younger bars were hosted by galaxies of later types. However, the conclusions are hampered by the assumption that the color traces the age, and that the metallicity effect or dust extinction are not important. Clearly, a study attempting to break the age-metallicity degeneracy and taking into account the effect of dust is needed. In addition, the influence of dust in the line-strength indices have been shown to be minimum (MacArthur 2005) while ages and metallicities from broad-band colors are heavily affected by dust as well as by the age-metallicity degeneracy.

To date, it has been difficult to obtain stellar population parameters along the bar because a high signal-to-noise ratio is required for this analysis. Although bars are high surface brightness structures, this still implies long integration times on medium-size telescopes. Recent work has presented the radial distribution of line-strength indices along bars (Pérez et al. 2007) for 6 galaxies.

The theoretical model of abundance gradients in bars developed by Friedli (1994) predicted, using $N$-body simulations of bars with pre-existing exponential abundances, a null evolution of the stellar abundance profile along the bar (although with a decrease in the mean metallicity) while the gas abundance profile flattened rapidly. Studies of the radial gas-phase abundance distribution have shown that there is, indeed, a flattening in the gas abundance along the bar (Martin \& Friedli 1997, 1999). However, recent work (Pérez et al. 2007) has shown, from stellar absorption line-strengths, that some bars present stellar metallicity gradients opposite to those found in the disks (i.e. more metal rich population at the end of the bar than in the regions of the bar closer to the center), contradicting the numerical results. This surprising result needs a more systematic study of the stellar population along the bars, properly comparing the line-strength indices of a larger sample of galaxies with the stateof-the art stellar populaton models to derive and quantify the variations of age and metallicity along the bar.

In this paper, we present a detailed stellar population analysis of the bar region of a sample of 20 early-type spirals (from $\mathrm{S} 0$ to $\mathrm{Sbc}$ ) with and without nuclear activity in order to derive ages, metallicities and relative chemical abundance ratios. The sample selection is described in Sect. 2. The observations and data reduction are presented in Sect. 3. The velocity dispersion measurements and the emission correction applied to the data are explained in Sect. 4.1. The line-strength derivation is described in Sect. 4.2. The morphological characterization of the sample is described in Sect. 4.3. Section 4.4 gives a description of the methodology followed to derive the stellar population parameters. The results of the line-strength indices and the kinematics are presented in Sect. 5. In this work, we concentrate only on the stellar populations of the bar region. The results of the central indices, bulge and bulge gradients will be presented in the second paper of this series.

\section{Sample characterization}

We have selected barred galaxies from the Third Reference Catalogue of bright galaxies (RC3) (de Vaucouleurs 1948) with the following criteria; to be classified as barred and with inclinations between $10^{\circ}$ and $70^{\circ}$, and nearby $\left(c z \leq 4000 \mathrm{~km} \mathrm{~s}^{-1}\right)$ to be able to properly resolve the bar. The sample is biased towards early-types barred galaxies, which have higher surface brightness, to ensure a high signal-to-noise ratio $(\mathrm{S} / \mathrm{N})$, crucial for a reliable determination of line-strength indices. Since we also aim to relate the mean stellar ages and metallicities of the bar to the nuclear activity in the center of the galaxy, half of the galaxies were selected to have nuclear activity. Eight of the galaxies present nuclear bars. Our final sample comprises 20 galaxies. It is, by no means, a statistically complete sample, but it is a representative sample of the early-type galaxy bar population. Table 1 shows the main properties of the sample as taken from the Hyperleda galaxy catalog (Paturel et al. 2003) ${ }^{1}$. The bar strength shown in Table $1^{2}$ have been taken from the literature, where strength is defined as the torque of a bar embedded in its disk (Combes \& Sanders 1981), see Table 1 for the references for the individual galaxies. The nuclear types have been obtained from Verón-Cetty \& Véron (2006). The sample shows a wide distribution of maximum rotational velocities $\left(80-260 \mathrm{~km} \mathrm{~s}^{-1}\right)$.

\section{Observations and data reduction}

We obtained long-slit spectra along the bar in our sample of 20 barred galaxies. The bar position angles were derived using the Digital Sky Survey (DSS) images.

\footnotetext{
1 http://leda.univ-lyon $1 . \mathrm{fr}$

2 Bar strength is shown here as bar-class; for assignment of bar-class to a certain bar strength, see Buta \& Block (2001), it is on a scale from 0 to 6,6 being the strongest bar.
} 
Table 1. General properties of the sample.

\begin{tabular}{|c|c|c|c|c|c|c|c|c|}
\hline Object & $v\left(\mathrm{~km} \mathrm{~s}^{-1}\right)$ & type & Bar-class ${ }^{1}$ & Nuclear type & Inner morph. ${ }^{2}$ & $B$ & $V_{\text {max,gas }}\left(\mathrm{km} \mathrm{s}^{-1}\right)^{3}$ & $i(\mathrm{deg})$ \\
\hline NGC $1169^{a}$ & 2387 & $\mathrm{SABb}$ & 3 & - & - & 12.35 & $259.1 \pm 7.3$ & 57.1 \\
\hline NGC 1358 & 4028 & $\mathrm{SAB}(\mathrm{R}) 0$ & - & Sy2 & - & 13.19 & $136.1 \pm 10.6$ & 62.8 \\
\hline NGC $1433^{b}$ & 1075 & (R)SB(rs)ab & 4 & Sy2 & Double-bar ${ }^{a}$ & 10.81 & $85.1 \pm 2.4$ & 68.1 \\
\hline NGC $1530^{b}$ & 2461 & $\mathrm{SBb}$ & 6 & - & - & 12.50 & $169.1 \pm 3.5$ & 58.3 \\
\hline NGC $1832^{d}$ & 1939 & $\mathrm{SB}(\mathrm{r}) \mathrm{bc}$ & 2 & - & - & 12.50 & $129.9 \pm 2.0$ & 71.8 \\
\hline NGC 2217 & 1619 & (R)SB(rs)0/a & - & LINER? & Double-bar $^{b}$ & 11.36 & $183.4 \pm 9.2$ & 30.7 \\
\hline NGC $2273^{c}$ & 1840 & $\mathrm{SB}(\mathrm{r}) \mathrm{a}$ & 2 & Sy2 & - & 12.62 & $192.2 \pm 5.5$ & 57.3 \\
\hline NGC 2523 & 3471 & SBbc & - & - & - & 12.64 & $211.4 \pm 10.9$ & 61.3 \\
\hline NGC 2665 & 1734 & (R)SB(r)a & - & - & - & 12.47 & $130.9 \pm 7.1$ & 32.8 \\
\hline NGC $2681^{c}$ & 692 & (R)SAB(rs)0/a & 1 & Sy3 & Triple-bar ${ }^{c}$ & 11.15 & $87.5 \pm 6.7$ & 15.9 \\
\hline $\mathrm{NGC} 2859^{c}$ & 1687 & $(\mathrm{R}) \mathrm{SB}(\mathrm{r}) 0^{\wedge}$ & 1 & Sy & Double-bar ${ }^{d}$ & 11.86 & $238.5 \pm 13.3$ & 33.0 \\
\hline NGC 2935 & 2271 & (R)SAB(s)b & - & - & - & 12.26 & $188.3 \pm 2.0$ & 42.7 \\
\hline NGC 2950 & 1337 & $(\mathrm{R}) \mathrm{SB}(\mathrm{r}) 0^{\wedge} 0$ & - & - & Double-bar $^{e}$ & 11.93 & - & 62.0 \\
\hline NGC 2962 & 1966 & (R)SAB(rs)0 & - & - & Double-bar ${ }^{c}$ & 12.91 & $202.9 \pm 9.9$ & 72.7 \\
\hline NGC $3081^{c}$ & 2391 & (R)SAB(r)0/a & 3 & Sy2 & Double-bar ${ }^{d}$ & 12.89 & $99.9 \pm 4.0$ & 60.1 \\
\hline $\mathrm{NGC} 4245^{c}$ & 815 & $\mathrm{SB}(\mathrm{r}) 0 / \mathrm{a}$ & 2 & & - & 12.33 & $113.5 \pm 5.4$ & 56.1 \\
\hline NGC $4314^{a}$ & 963 & $\mathrm{SB}(\mathrm{rs}) \mathrm{a}$ & 3 & LINER & Double-bar ${ }^{c}$ & 11.42 & $253.3 \pm 24.6$ & 16.2 \\
\hline NGC $4394^{d}$ & 922 & $(\mathrm{R}) \mathrm{SB}(\mathrm{r}) \mathrm{b}$ & 3 & LINER & - & 11.59 & $212.5 \pm 16.0$ & 20.0 \\
\hline $\mathrm{NGC} 4643^{c}$ & 1335 & $\mathrm{SB}(\mathrm{rs}) 0 / \mathrm{a}$ & 3 & LINER & - & 11.68 & $171.4 \pm 7.2$ & 42.9 \\
\hline NGC $5101^{d}$ & 1868 & $(\mathrm{R}) \mathrm{SB}(\mathrm{r}) 0 / \mathrm{a}$ & 2 & - & - & 11.59 & $195.7 \pm 9.0$ & 23.2 \\
\hline
\end{tabular}

(1) : ${ }^{a}$ Bar class derived from the $K$-band light distribution, Block et al. (2001); ${ }^{b}$ Bar class derived from the $K$-band light distribution, Block et al. (2004); ${ }^{c}$ Bar class derived from the $K$-band light distribution, Buta et al. (2006); ${ }^{d}$ Bar class derived from the $H$-band light distribution, Laurikainen et al. (2004). (2) : ${ }^{a}$ Buta (1986); ${ }^{b}$ Jungwiert (1997); ${ }^{c}$ Erwin (2004); ${ }^{d}$ Wozniak (1995). (3) Rotational velocity corrected for inclination.

The observations were performed in two different runs. In the first run (run1 hereafter), spectroscopy of 6 galaxies was obtained at the $2.3 \mathrm{~m}$ telescope at Siding Spring Observatory (RSAA, ANU, Australia) during February 2006 with the Double Beam Spectrograph (DBS, Rodgers et al. 1998). The gratings $600 \mathrm{~B}$ and the $600 \mathrm{R}$ were used for the blue and red arms, respectively, with a slit width of 2 arcsec. This set-up gives a dispersion of $1.1 \AA$ pixel $^{-1}$ for the blue arm and $1.09 \AA$ pixel $^{-1}$ for the red arm in the wavelength intervals $3892-5814 \AA$ and 5390-7314 ̊ respectively, and a final spectral resolution of $F W H M \sim 2.2 \AA$. One of the main problems in the derivation of accurate line-strength indices in regions of low surface brightness comes from the systematic effects due to sky subtraction. One of the advantages of the DBS is the length of the slit (6.7 arcmin) which allows the selection of sky regions at large radii, not contaminated by the light of the galaxy.

The second set of observations (run2 hereafter) were carried out in February 2007 at El Roque de Los Muchachos Observatory (La Palma, Spain). We used IDS mounted on the Isaac Newton telescope $(2.5 \mathrm{~m})$. The R632V grating combined with a $1.5 \operatorname{arcsec}$ slit width gives a resolution of $3 \AA$ (FWHM). The full unvignetted slit length in IDS is 3.3 arcmin. In order to obtain enough sky coverage on one of the galaxy sides, we displaced the center of the slit from the galaxy center during the observations.

Comparison arc lamp exposures were obtained in both runs to wavelength calibrate the frames. Spectrophotometric standards were observed with a slit-width of 6 arcsec in order to avoid differential flux losses due to atmospheric diffraction. Additionally, we observed 11 and 35 G-K stars in the first and second run respectively from the Lick/IDS (Gorgas et al. 1993; Worthey et al. 1994) and MILES libraries (Sánchez-Blázquez et al. 2006) in order to calibrate our data into the Lick/IDS spectrophotometric system. The total integration time for each galaxy is listed in Table 2. Only the blue part of the spectra in
Table 2. Log of observations.

\begin{tabular}{cllll}
\hline \hline Object & Telescope & Date & $\begin{array}{l}\text { PA } \\
\text { (deg) }\end{array}$ & $\begin{array}{l}\text { Exp. } \\
\text { time }\end{array}$ \\
\hline NGC 1169 & INT & $09 / 02 / 2007$ & 95 & $3 \mathrm{~h}$ \\
NGC 1358 & INT & $10,11 / 02 / 2007$ & 127 & $3 \mathrm{~h}$ \\
NGC 1433 & $2.3 \mathrm{~m}$, SSO & $03,06 / 02 / 2006$ & 96 & $2.5 \mathrm{~h}$ \\
NGC 1530 & INT & $08 / 02 / 2007$ & 121 & $3 \mathrm{~h}$ \\
NGC 1832 & INT & $12 / 02 / 2007$ & 171 & $3 \mathrm{~h}$ \\
NGC 2217 & $2.3 \mathrm{~m}$, SSO & $6 / 02 / 2006$ & 290 & $2 \mathrm{~h}$ \\
NGC 2273 & INT & $07 / 02 / 2007$ & 116 & $3 \mathrm{~h}$ \\
NGC 2523 & INT & $09 / 02 / 2007$ & 118 & $3 \mathrm{~h}$ \\
NGC 2665 & $2.3 \mathrm{~m}$, SSO & $01,02 / 02 / 2006$ & 54 & $3.3 \mathrm{~h}$ \\
NGC 2681 & INT & $02 / 02 / 2007$ & 28 & $3 \mathrm{~h}$ \\
NGC 2859 & INT & $08 / 02 / 2007$ & 162 & $3 \mathrm{~h}$ \\
& & $12 / 02 / 2007$ & 72 & $3 \mathrm{~h}$ \\
NGC 2935 & $2.3 \mathrm{~m}$, SSO & $5,6 / 02 / 2006$ & 135 & $3 \mathrm{~h}$ \\
NGC 2950 & INT & $11 / 02 / 2007$ & 162 & $3 \mathrm{~h}$ \\
NGC 2962 & INT & $10 / 02 / 2007$ & 175 & $3 \mathrm{~h}$ \\
NGC 3081 & $2.3 \mathrm{~m}$, SSO & $01 / 02 / 2006$ & 64 & $2.5 \mathrm{~h}$ \\
NGC 4245 & INT & $07,08 / 02 / 2007$ & 135 & $3 \mathrm{~h}$ \\
NGC 4314 & INT & $09 / 02 / 2007$ & 147 & $2.5 \mathrm{~h}$ \\
& & $12 / 02 / 2007$ & 237 & $3 \mathrm{~h}$ \\
NGC 4394 & INT & $11 / 02 / 2007$ & 143 & $3 \mathrm{~h}$ \\
NGC 4643 & INT & $10 / 02 / 2007$ & 134 & $3 \mathrm{~h}$ \\
& $2.3 \mathrm{~m}$, SSO & $1,5,6 / 02 / 2006$ & 134 & $3.3 \mathrm{~h}$ \\
NGC 5101 & $2.3 \mathrm{~m}$, SSO & $3,5,6 / 02 / 2006$ & 122 & $3 \mathrm{~h}$ \\
\hline
\end{tabular}

the case of the DBS observations is analyzed in this paper. The red part of the spectra, i.e. the nebular emission properties, will be presented elsewhere.

The reduction of the two runs has been carried out with the package REDUCEME (Cardiel 1999). Standard data reduction 
procedures (flat-fielding, cosmic ray removal, wavelength calibration, sky subtraction and fluxing) were performed. Error images were created at the beginning of the reduction and were processed in parallel with the science images. Initial reduction of the CCD frames involved bias subtraction and removal of pixel-to-pixel sensitivity variations (using flat-field exposures of a tungsten calibration lamp). Correction of a two-dimensional low-frequency scale sensitivity variations was done using twilight sky exposures. Arc frames were used to convert the spectra into a linear wavelength scale. We typically use $\sim 60$ lines. The lines were fitted with a 1st order polynomia, with rms errors lower than $0.1 \AA$. Atmospheric extinction was calculated using the extinction curve of King (1985). To correct the effect of interstellar extinction, we used the curve of Cardelli et al. (1989). The reddenings applied were extracted from the RC3 catalogue of galaxies (de Vaucouleurs 1948). Relative flux calibration was achieved using the spectra of the observed standard stars. All the flux calibration curves of each night were averaged and the flux calibration errors were estimated from the differences between the indices measured with different curves. After correction for geometrical distortions (both spatial and spectral), a sky image was generated for each observation by fitting, for each channel, a low order polynomial using regions selected from both sides of the galaxy frame in the first run, and only from one of the sides of the frame for the galaxies in the second run.

In order to derive the index spatial distribution for the fully reduced galaxy frames, a final frame was created by extracting spectra along the slit, binning in the spatial direction to guarantee a minimum signal-to-noise ratio of 20 per $\AA$ in the spectral region of $\mathrm{Mgb}$. This minimum $\mathrm{S} / \mathrm{N}$ ensures errors lower than $15 \%$ in most of the Lick/IDS indices (Cardiel et al. 1998).

\section{Analysis}

\subsection{Velocity dispersion measurements and emission correction}

Whenever ionized gas is present in the galaxy, emission lines can affect the measurement of the absorption indices. In particular, all the Balmer lines $(\mathrm{H} \delta, \mathrm{H} \gamma$ and $\mathrm{H} \beta), \mathrm{Fe} 5015$, and Mgb indices are affected by the presence of $\mathrm{H} \delta \lambda 4101, \mathrm{H} \gamma \lambda 4340, \mathrm{H} \beta \lambda 4861$, [OIII] $] \lambda$ 4959, 5007, and [NII] $\lambda \lambda 5198,5200$ lines in emission. The presence of emission in the Balmer lines tend to fill the absorption profiles, making the indices weaker. If this is not corrected properly, older ages can be artificially measured. In order to correct for the emission line contamination we have used the routine GANDALF (Sarzi et al. 2005, S05 hereafter). This routine simultaneously fits the stellar and the emission line spectra, by treating the emission lines as additional Gaussian templates and iteratively searching for the best radial velocity and velocity dispersion. To model the stellar spectra, we use the stellar population models by Vazdekis et al. (2008) based on the MILES stellar library (Sánchez-Blázquez et al. 2006), previously degraded to our instrumental resolution. The continuum shape of the stellar templates and that of the galaxies is fitted and removed using a multiplicative Legrende polynomial and subtracted from all spectra.

As explained in S05, despite that the kinematics of the different emission lines can be fitted independently, in some cases, as in $\mathrm{H} \beta$, degeneracies between the stellar and emission templates can cause spurious detections. Furthermore, it is very difficult to constrain the kinematics of the weak emission lines. For these reasons, we follow the same procedure as in S05 and impose that all the emission lines have the same kinematics as the strong line
[OIII] $\lambda 5007$, except for the cases when $\mathrm{H} \beta$ line is very strong. The steps followed are:

(1) masking the emission lines and calculating a first estimate of the stellar velocity and velocity dispersion;

(2) convolving our templates with the calculated velocity dispersion and shifting them according to the recessional velocity, and then calculating the kinematics of the [OIII] $\lambda 5007$ lines;

(3) we force all the emission lines to have the same kinematics as the one calculated with [OIII] $\lambda 5007$ and fit, simultaneously, emission and absorption spectra.

Figure 1 shows the central spectrum of NGC 1358, a galaxy with particularly strong emission, before and after cleaning the emission using the method described above. In some cases (i.e. in the nuclei of active galaxies), the emission lines cannot be fitted with a single Gaussian, as the emission line profiles show asymmetries. In those cases, double Gaussians are fitted in order to reproduce the observed profile. This process will be explained in more detail in a future paper, when we analyze the properties of the bulges and central parts in our sample (Pérez et al. 2009, in preparation).

GANDALF makes use of PPXF (penalized pixel-fitting) to calculate the stellar kinematics. PPXF is a software described in Capellari \& Emsellem (2004). It is specially appropriate for low signal-to-noise data or when the data is not well sampled. The errors in the radial velocity and velocity dispersion were computed through numerical simulations. In each simulation, a bootstrapped galaxy spectrum, obtained using the error spectrum provided by the reduction with REDUCEME, is fed into the algorithm. Errors in the parameters are then calculated as the unbiased deviation of the different solutions. The kinematics of the gas and the diagnostic emission lines will be subject of a future paper (Florido et al. 2009, in preparation).

\subsection{Line-strength indices}

Lick/IDS line-strength indices using the definition in Trager et al. (1998) are measured in all the lines in binned spectra, cleaned of emission, along the radius. The errors are calculated from the uncertainties caused by photon noise, wavelength calibration and flux calibration.

Line-strength indices depend on the broadening of the lines caused by instrumental resolution and by the internal motion of the stars. Therefore, when comparing with the stellar population models, the synthetic and observed indices need to be measured at the same resolution.

We are using two different sets of models to derive the synthetic indices. The first one, from Vazdekis et al. (2008), is built using a library (MILES) with constant resolution along the wavelength direction $(2.3 \AA)$ which is also flux calibrated (Sánchez-Blázquez et al. 2006). The main advantage of these models is that they predict the whole spectrum for a population of a given age and metallicity and, therefore, it can be convolved with a broadening function to mimic the desired resolution. To compare with these models we degrade all the spectra to the resolution of the run 1 spectra $(F W H M \approx 5.2 \AA)$.

The second set of models we use is that of Thomas et al. (2003, TMB03 hereafter). These models are based on the Lick/IDS fitting functions (Gorgas et al. 1993; Worthey et al. 1994) and, therefore, their resolution cannot be modified. To compare our measurements with this model we degrade our spectra to the wavelength-dependent resolution of the Lick stars following the prescriptions given in Worthey \& Ottaviani (1997). 


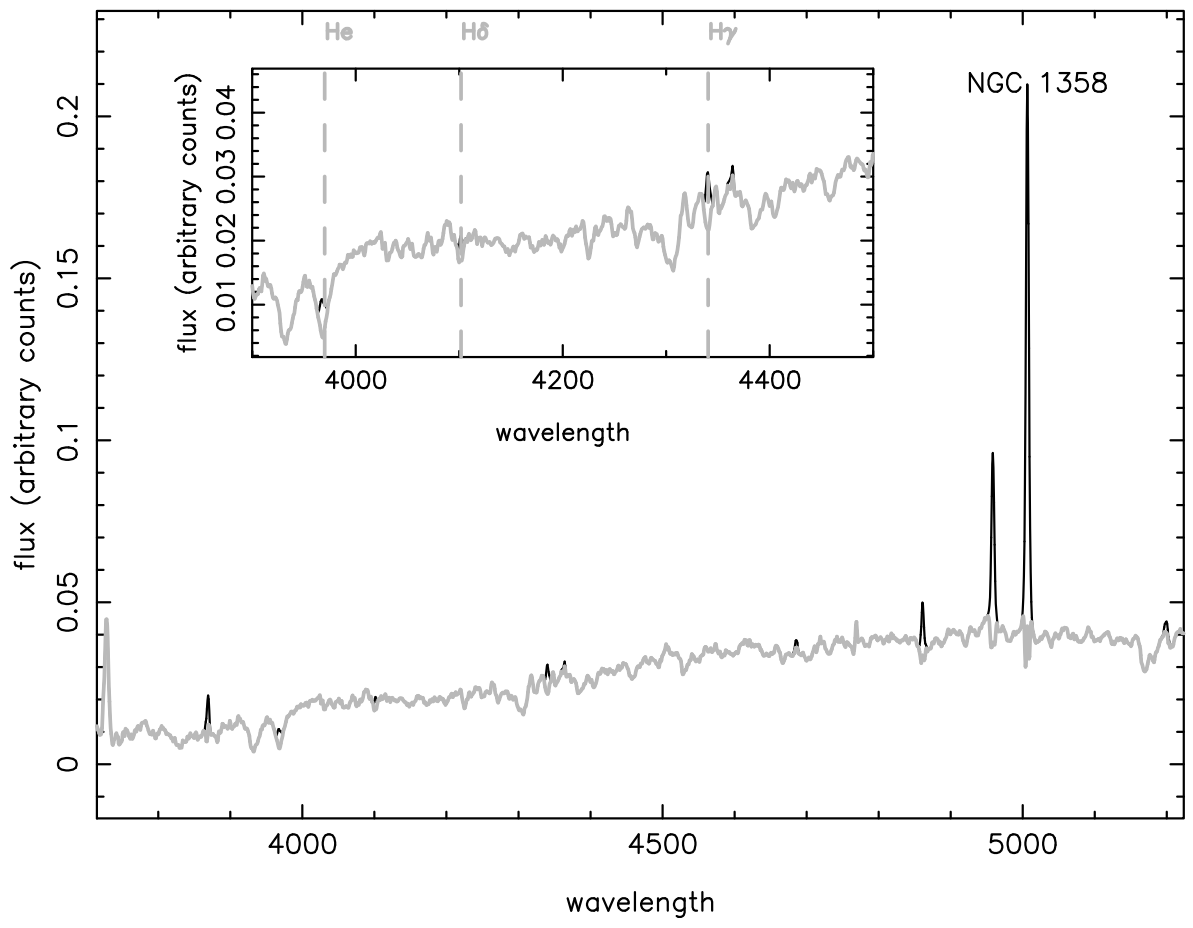

Fig. 1. Example of the emission correction with GANDALF for the central spectrum of NGC 1358. The grey line shows the spectrum after being cleaned of emission lines, while the black spectra show the spectrum before. NGC 1358 is a galaxy from the sample with particularly strong emission lines; the fitting process applied to correct for the emission does a very good job even in this extreme case (Sect. 4.1 for details).
Absorption lines are not only broadened by the line-spread function of the instrument, but also by Doppler broadening due to the velocity dispersion of the stars. To compare with the models and with galaxies with different velocity dispersions, this needs to be corrected. Because the variation of the indices with the velocity dispersion depends on the strength of the index itself (e.g. Kuntschner 2000), one should perform a different correction for each individual spectrum along the radius. To calculate these corrections we use the template obtained in GANDALF when fitting the spectra in the first bin, i.e. the bar-bin closest to the galaxy center. We degrade this spectrum at the Lick/IDS resolution and broaden it to different velocity dispersions in steps of $10 \mathrm{~km} \mathrm{~s}^{-1}$. We then build polynomia (for each individual spectrum) of the form: $C(\sigma)=I(\sigma=0) / I(\sigma)$ for atomic indices, and $C(\sigma)=I(\sigma=0)-I(\sigma)$ for molecular indices, where $I(\sigma=0)$ represents the index at the Lick resolution and $I(\sigma)$, the index at Lick resolution broadened with a velocity dispersion $\sigma$. Polynomia are built as well with the initial broadening equal to the instrumental resolution of run1, in order to compare with the V08 models at the resolution of the data. We finally apply a single correction to each galaxy using the polynomia derived for the spectra at the beginning of the bar, and use the rest of the templates along the bar to calculate the error for this approximation. This error is added in quadrature to the final index error.

Once the indices in the galaxy spectra and models are measured at the same resolution and corrected by the velocity dispersion broadening, a further correction is needed in order to fully transform the measurements into the right spectrophotometric system. These small corrections try to compensate for the differences between the models and the data that appear due to a deficient calibration of the Lick/IDS stellar spectra. To obtain these offsets, we compare the index measurements in our sample of stars in common with the Lick/IDS library observed for this purpose. The final offsets are very small and are listed in Table B.1. Figures with the comparison of individual indices can be found in Appendix B. In principle, because our data are relatively flux calibrated, a further offset is not necessary to transform our measurements into the MILES spectrophotometric system (that is also flux calibrated). However, we also compare the indices measured in stars in common with this library. As expected, for most of the indices the offsets are null (see Table B.1), although small offsets were found for some of them.

\subsection{Morphological characterization}

In order to relate the stellar population properties to the morphological features, we perform an ellipse fitting analysis on available archived $R$-band images of the galaxy sample. For nine out of the 20 galaxies, Sloan Digital Sky Survey ${ }^{3}$ (SDSS) data was available. For NGC 1530 we use the data published in Zurita \& Pérez (2008), for NGC 2273 the $R$-band image from Hameed \& Devereux (1999) and for NGC 1169 the $R$-band from Knapen et al. (2004). Ellipse fitting to the light distribution is performed using the ELLIPSE task within IRAF. As all the galaxies showed point-like nuclear regions, the center is fixed using the coordinates obtained by fitting a Gaussian to the nucleus. The position angle (PA) and the ellipticity $(\epsilon)$ are left as free parameters in the fitting. The minimum ellipticity in the bar region has been adopted as the definition for the size of the bar. It has been shown (Michel-Dansac \& Wozniak 2006) that this measurement is correlated with the corotation radius and, therefore, gives a bar structural size; it is also the method to calculate the bar size that is the least sensitive to dust absorption. Furthermore, it is a very simple criterion to reproduce and can be compared to other results in the literature. For a discussion about the differences in the bar size obtained using different methods see for example Erwin (2005). The bulge radius is considered to be the radius where the PA and ellipticity $\epsilon$ start showing a typical bar profile, i.e. increasing $\epsilon$ and constant PA. Figures 2 and 3 show the results of the ellipse fitting for all the analyzed galaxies together with an $R$-band image, and an image of the residuals obtained subtracting the galaxy model from the $R$-band galaxy image. The radius of the bar, bulge and the extent of our spectrocopic data are overplotted on the two figures. For the galaxies for which

3 http://www.sdss.org 
NGC 1169
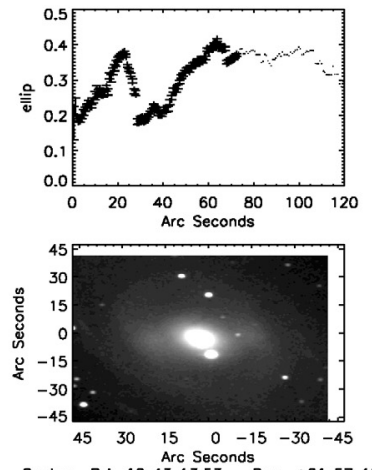

Center: R.A. 124313.53 Arcoc +015710.0

NGC 2273
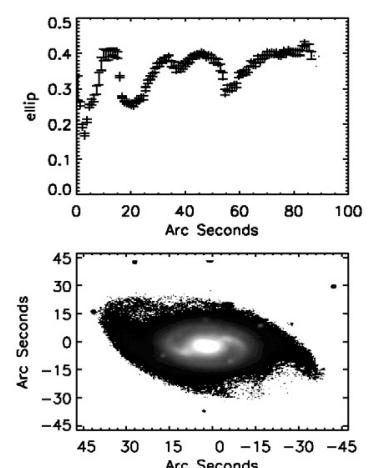

Center: R.A. 124313.53 Dec +015710.0

NGC 2859
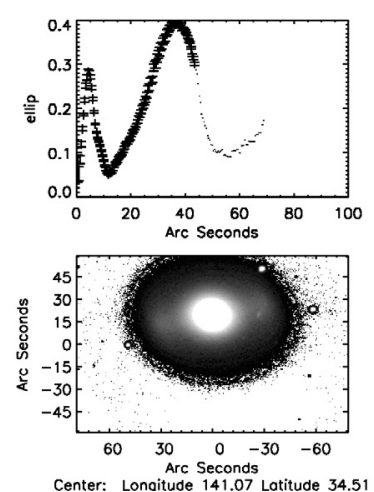
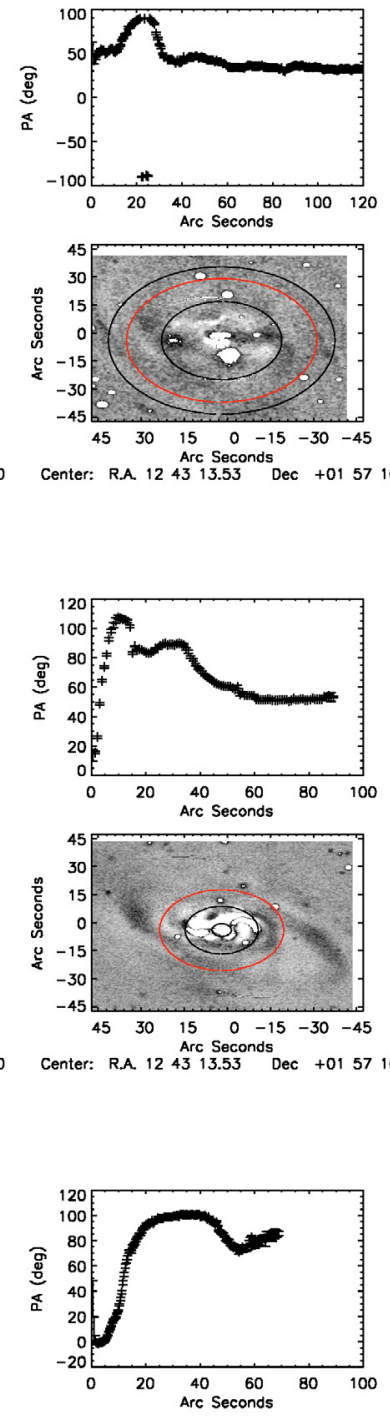

Center: R.A. 124313.53 Dec +015710.0
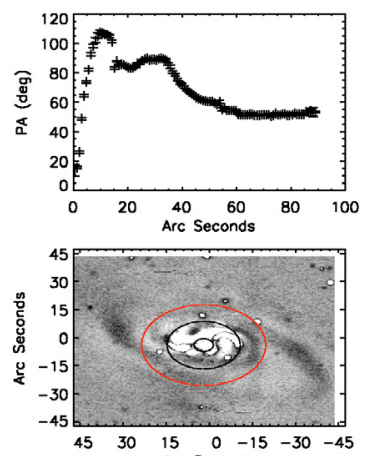

Arc Seconds
Dec +015710.0

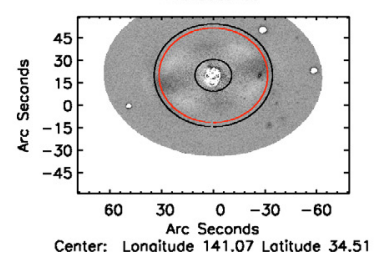

NGC 1530
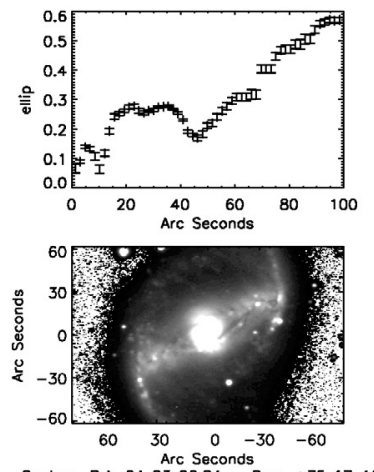

Center: R.A. 042326.84 Aec +751741.2
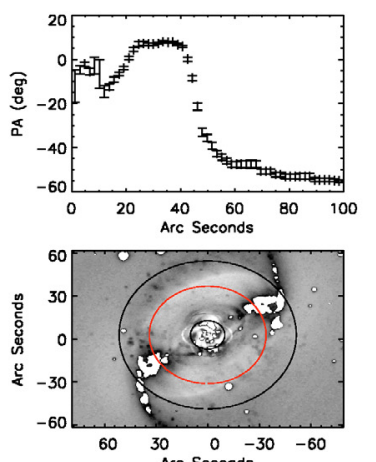

Center: R.A. 042326.84 Aec +751741.2
NGC 2681
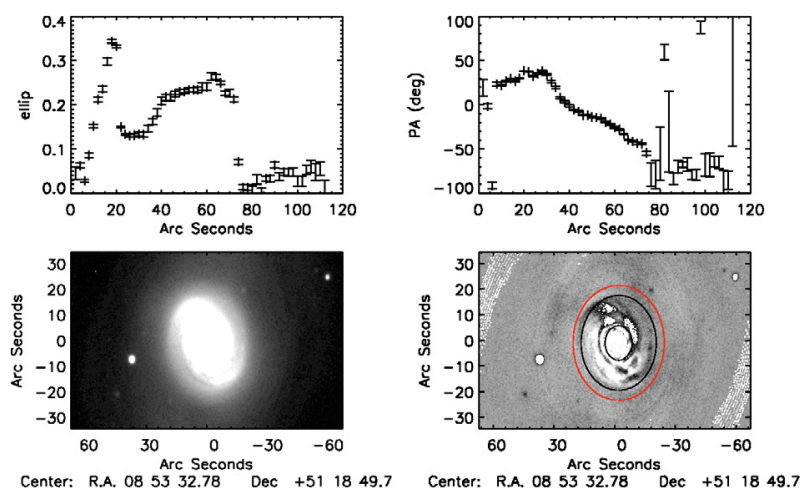

NGC 2950
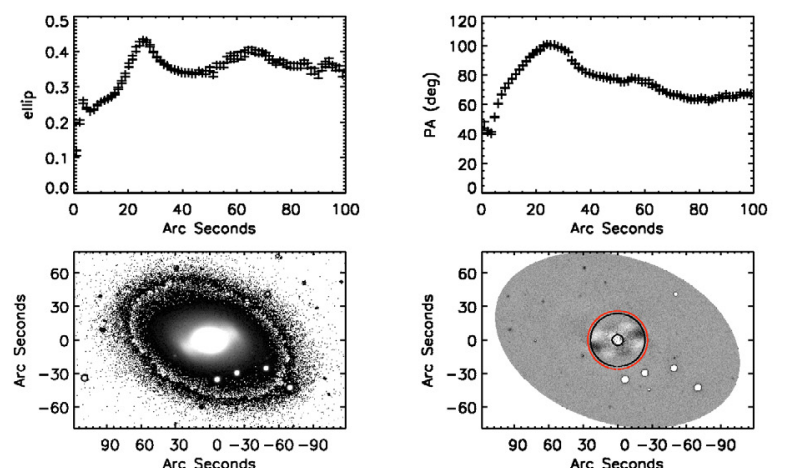

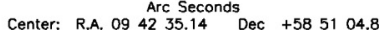

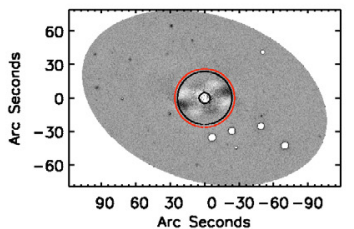

Center: R.A. 094235.14 Dec +585104.8

Fig. 2. Morphological analysis of the sample galaxies from SDSS images. For each galaxy we show the ellipticity distribution, position angle distribution, the $R$-band image and the residual image. The red circle shows the extent of the data and the black circle the position of the bar end.

no SDSS data was available, Fig. 4 shows 2MASS data together with the spectroscopic data extent. Table 3 shows the derived bar size for the galaxies, with good imaging data from the SDSS and the literature, in comparison with the bar sizes obtained by other authors. There is good agreement between the values. The beginning of the bar coincides, in many cases, with a change in the trends of the line strength indices with radius (see Sect. 4.4), giving confidence in our size estimations. The general morphology of the galaxies in the sample shows a large range of properties. Table 1 shows the detailed morphological types of all the galaxies. Some galaxies, independently of their type, have outer rings, some host strong bars and some are of intermediate AB type. We, therefore, cover a wide range in general galaxy morphology. This allows us to test systematic behavior associated with different morphologies.

\subsection{Derivation of stellar population parameters}

As we mentioned before (Sect. 4.2), to derive stellar population parameters we compare our line-strength indices with two different sets of stellar population models: TMB03 and V08. TMB03 use the fuel consumption theorem (Renzini \& Buzzoni 1986) to evaluate the energetics of the post-main sequence phases. They use the stellar tracks of Cassisi et al. (1997), Bono et al. (1997) and Cassisi et al. (1999), and for the more metal rich models the ones by Salasnich et al. (2000). V08 uses the isochrones of Girardi et al. (2000) for all metallicities, and an empirical calibration to transform the models from the theoretical to the observational plane. By comparing with two different sets of models we take into account some of the uncertainties coming from 
NGC 2962
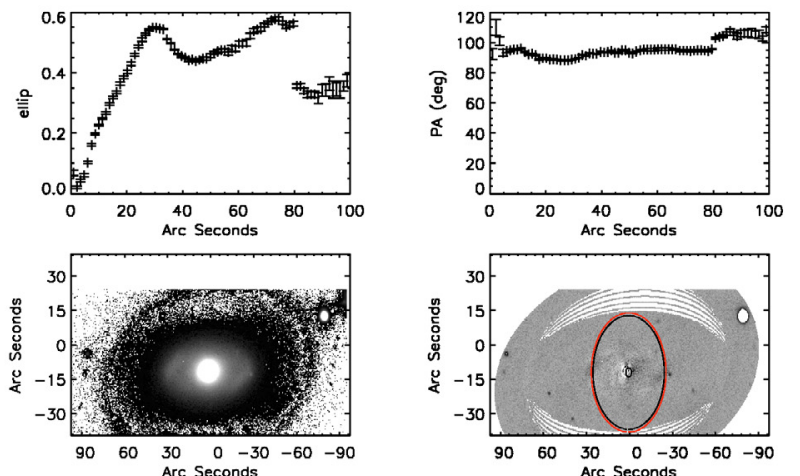

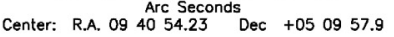

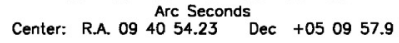

NGC 4314
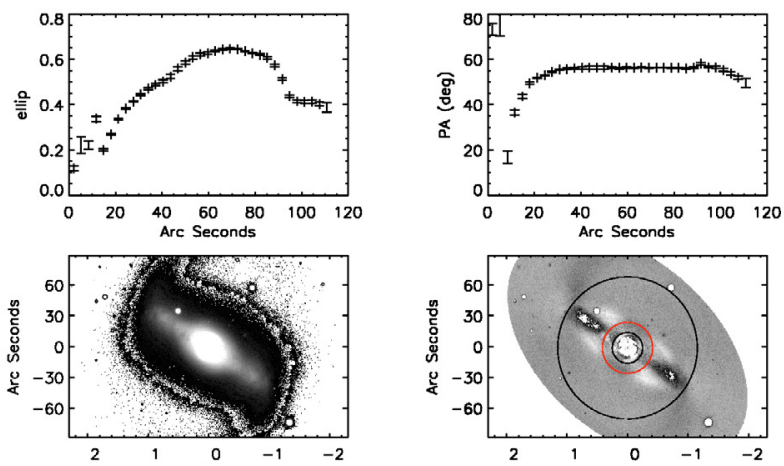

Center: R.A. 122232.06 Dec +295343.8

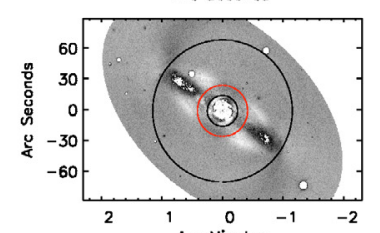

Center: R.A. 122232.06 Dec +295343.8

NGC 4643
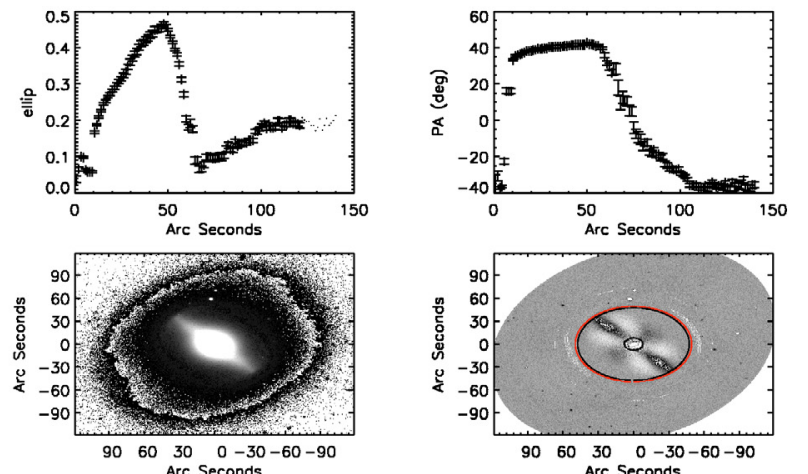

Center: R.A. 124320.16 Dec +015841.8

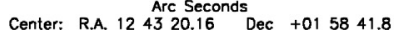

NGC 4245
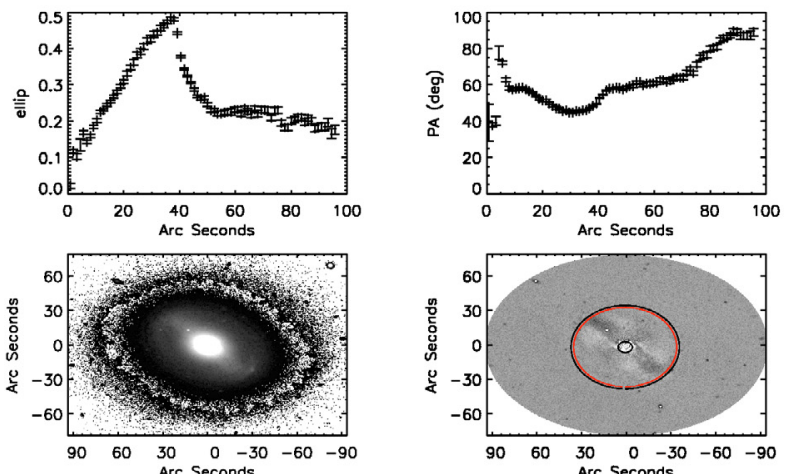

Center: R.A. 121736.81 Dec +293629 .

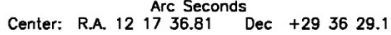

NGC 4394
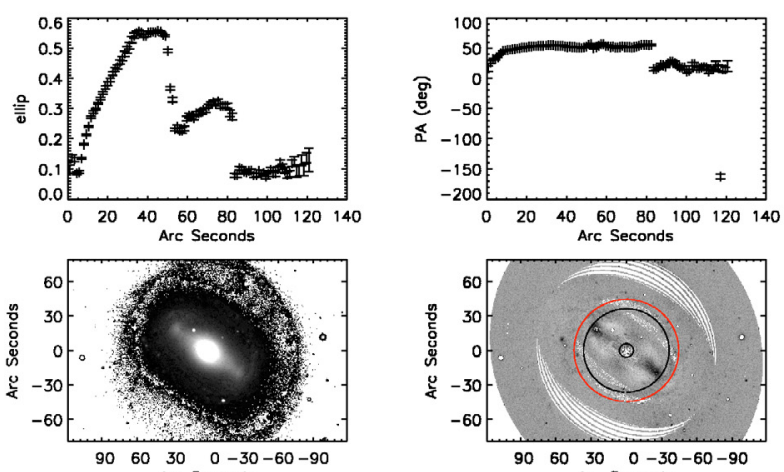

Center: R.A. 122555.67 Dec +181250.2

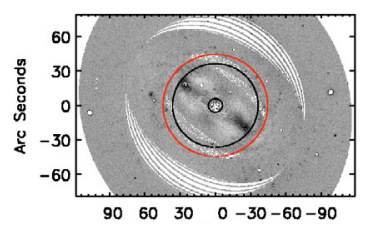

Center: R.A. 122555.67 Dec +181250.2

Fig. 3. Continuation of Fig. 2.

the variety of ingredients chosen by different authors. Below, we give a brief explanation of such possible uncertainties.

V08 models provide synthetic spectra of single stellar populations with a given age, metallicity and solar chemical abundances ratios (the ones of the empirical library). We know that, in some systems, the ratio between different elements does not match the solar values. For example, in giant elliptical galaxies, $[\mathrm{Mg} / \mathrm{Fe}]>0.0$ (e.g. O'Connell 1976; Faber et al. 1992). It is important to take this effect into account because the presence of differences in the chemical abundance partitions affects the age measurements (e.g. TMB03). Several groups have characterized the variation of line-strength indices for single stellar populations to variations of individual chemical abundances (e.g. Tantalo et al. 1998; Trager et al. 2000; Proctor \& Sansom 2002; TMB03). In order to do this, we follow the prescriptions given in Trager et al. (2000) with the corrections for negative indices given in Thomas et al. (2003) using the response functions by Korn et al. (2004). We make the assumption that all the $\alpha$-elements are equally enhanced with the exception of $\mathrm{Ca}$, which is assumed to follow Fe-peak element abundances. $\mathrm{C}$ is also assumed to be enhanced. Therefore, we define two groups: the enhanced group (C, N, O, Ne, Na, Mg, Al, Si, S and Ar) and the depressed group ( $\mathrm{Fe}, \mathrm{Ca}, \mathrm{Cr}$ and $\mathrm{Ni}$ ). This matches model 4 in Trager et al. (2000). We assume a linear relationship between the $[\mathrm{Z} / \mathrm{H}],[\mathrm{Fe} / \mathrm{H}]$ and $[\mathrm{E} / \mathrm{Fe}]$ (where E represents the mass of all elements with enhanced abundance ratios) of the 

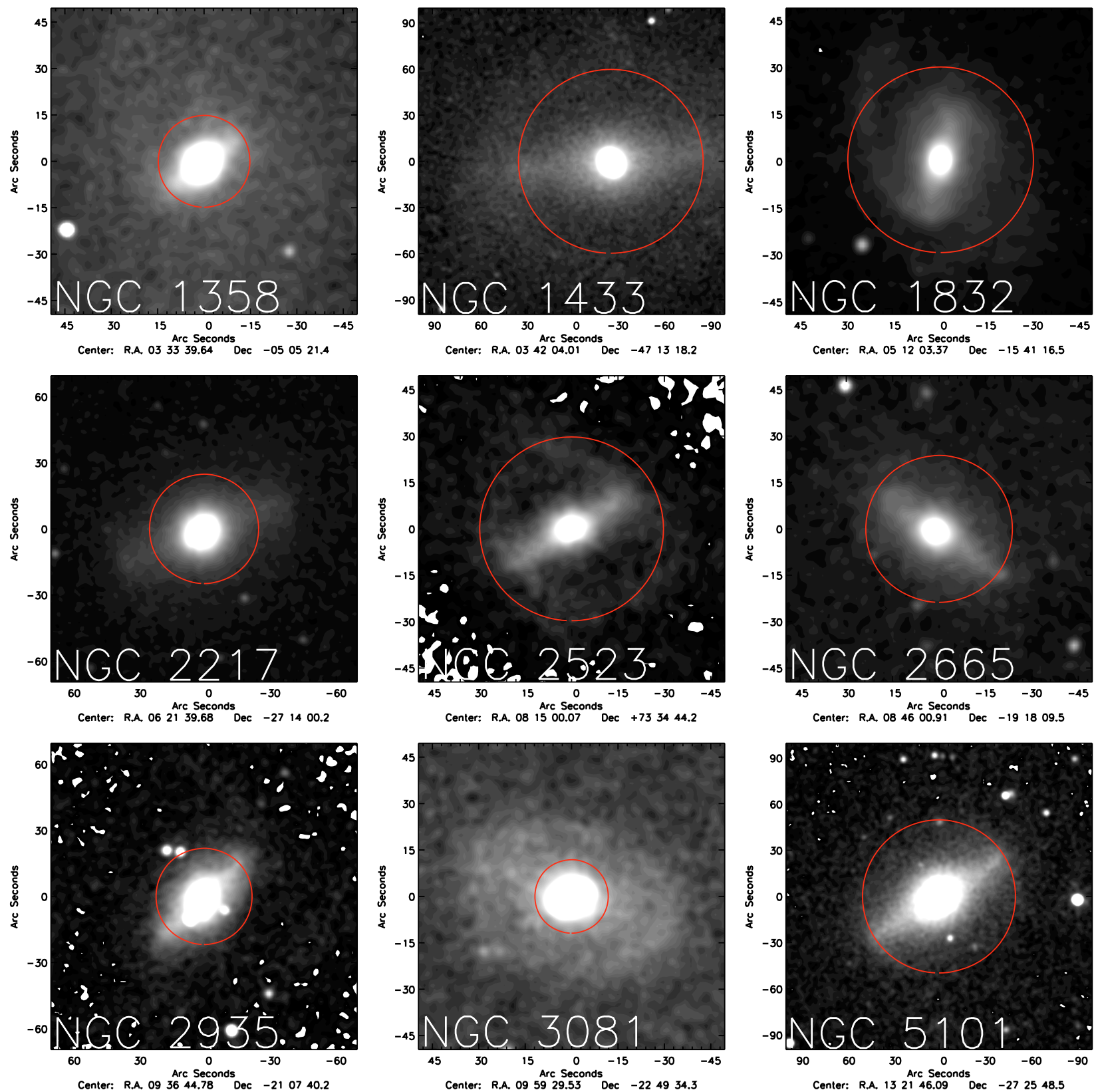

Fig. 4. 2MASS images of the galaxies without SDSS imaging. The red circles show the extent of the spectroscopic data.

form $[\mathrm{Z} / \mathrm{H}]=[\mathrm{Fe} / \mathrm{H}]+0.929[\mathrm{E} / \mathrm{Fe}]$ (see Trager et al. 2000) and all the variations of $[\mathrm{E} / \mathrm{Fe}]$ are compensated by variations of the Fe-peak group in such a way that $[\mathrm{Z} / \mathrm{H}]$ stay constant.

The changes of the indices to different chemical composition is calculated as (see Trager et al. 2000):

$\frac{\delta I}{I_{0}}=\prod_{i}\left(1+R_{0.3}\left(X_{i}\right)\right)^{\left[X_{i} / H\right] / 0.3}-1$

where $R_{0.3}\left(X_{i}\right)$ is the metallicity dependent response function by Korn et al. (2004) to a variation of $\left[\mathrm{X}_{i} / \mathrm{H}\right]$ of the element $i$.

The models of TMB03 already incorporate a similar treatment to take into account the differences in the chemical abundances ratios. We use the original version of the models with $[\mathrm{C} / \mathrm{Fe}]$ and $[\mathrm{N} / \mathrm{Fe}]=0$ and $[\mathrm{Ca} / \alpha]=0$, but this choice does not affect our results.
To obtain stellar population parameters we use 4 indices, namely $\mathrm{H} \delta_{\mathrm{A}}, \mathrm{H} \gamma_{\mathrm{A}}, \mathrm{Fe} 4383$ and $\mathrm{Mgb}$ and follow a multi-index approach as described in Proctor \& Samson (2002). We avoid using $\mathrm{H} \beta$ because this is the Balmer index most affected by the presence of emission. The errors are estimated by performing 100 Monte Carlo simulations where each index was perturbed by a Gaussian distribution with $\sigma$ equal to the index error.

The average deviations of the measured indices from the indices predicted by the model for the derived values of age, metallicity and $[\mathrm{E} / \mathrm{Fe}]$, are shown in Fig. C.1. Despite the fact that we have only used 4 indices for the fit, it can be seen that most of the indices are reproduced within the errors by the stellar population parameters derived with this method. Some of the indices that are poorly reproduced for some galaxies are the broad indices $\mathrm{Mg}_{1}, \mathrm{Mg}_{2}, \mathrm{CN}_{1}$ and $\mathrm{CN}_{2}$. This is understandable as those indices are very sensitive to the flux calibration. As we have 
Table 3. Bar sizes. The bar size derivation is described in Sect. 4.3 .

\begin{tabular}{|c|c|c|}
\hline Galaxy name & $\begin{array}{l}\text { Bar size (arcsec) } \\
\text { (other studies) }\end{array}$ & $\begin{array}{l}\text { Bar size }(\operatorname{arcsec}) \\
\text { (our study) }\end{array}$ \\
\hline NGC 1169 & - & 29 \\
\hline NGC $1358^{d}$ & 25 & - \\
\hline NGC $1433^{b, i}$ & 90,66 & - \\
\hline $\mathrm{NGC} 1530^{e}$ & 50 & 69 \\
\hline NGC $1832^{f}$ & 17 & - \\
\hline NGC $2217^{b ; f ; i}$ & $50,37,43$ & - \\
\hline NGC $2273^{a}$ & 14 & 21 \\
\hline NGC 2523 & - & - \\
\hline NGC 2665 & - & - \\
\hline NGC $2681^{1 ; a ; c ; g}$ & $50,25,29$ & 23 \\
\hline NGC $2859^{b ; c}$ & 34,46 & 48 \\
\hline NGC $2935^{f ; i}$ & 25,39 & - \\
\hline NGC $2950^{a ; g}$ & 24,38 & 44 \\
\hline NGC $2962^{a}$ & 29 & 45 \\
\hline NGC $3081^{b ; g ; c}$ & $35,41,38$ & 35 \\
\hline NGC $4245^{a}$ & 37 & 59 \\
\hline NGC $4314^{a, g}$ & 67,75 & 92 \\
\hline NGC $4394^{h ; i}$ & 52,35 & 56 \\
\hline NGC $4643^{a}$ & 50 & 67 \\
\hline NGC $5101^{f}$ & 50 & - \\
\hline
\end{tabular}

(1) NGC 2681 is a triple barred system. Erwin (2005) gives the value of the primary bar, while we give here the value of the secondary bar. ${ }^{a}$ Erwin (2005) bar size given as the radius of maximum ellipticity in the bar region; ${ }^{b}$ Erwin (2004) bar size given at the radius of maximum ellipticity in the bar region; ${ }^{c}$ Wozniak (1995); they give the projected bar size at the radius of minimum ellipticity; ${ }^{d}$ Mulchaey (1997); they give the bar size as the radius at the minimum of ellipticity; ${ }^{e}$ Regan (1996); ${ }^{f}$ Jungwiert (1997), bar size given as the radius of maximum ellipticity in the bar region; ${ }^{g}$ Friedli et al. (1996); they give the projected bar size as the radius at the ellipticity minimum; ${ }^{h}$ Gadotti \& de Souza (2006), bar size given as the radius of maximum ellipticity in the bar region; ${ }^{i}$ Martin \& Roy (1995), bar size given by visual inspection of photographic plates.

not corrected for internal extinction in the galaxy, the shape of the continuum in our spectra is affected by reddening. For the rest of the indices, the effect of dust is almost negligible (see, e.g. MacArthur 2005). In order to analyze the possible correlations between the results from the stellar population analysis and other parameters, we fit a slope to the derived age, metallicity and $[\mathrm{E} / \mathrm{Fe}]$ distributions with a simple linear regression weighted by the errors in the $y$-direction. Table 5 shows the fitted slope together with the errors. For four galaxies out of the 20, the obtained gradients are not reliable due to large fitting errors and these galaxies are discarded in the plots and the analysis regarding the gradient values (NGC 1530, NGC 3081, NGC 4314, NGC 2935); we include them in Table 5.

To check the dependence of our results on the choice of stellar population model, we repeat the calculations using the new models by V08. Figure 5 shows the comparison of the age, metallicity and $[\mathrm{E} / \mathrm{Fe}]$ gradients (the slope of the fit) using different models. As can be seen, the comparison is very good in general. We have checked that none of our results is affected by our choice of model.

An important concern about the obtained results is that a non-perfect emission correction could still be affecting our derived indices. The effect of a poor emission correction is to make the age older and, therefore, because the errors in age and metallicity are correlated, the metallicity is lowered. It is difficult to estimate an error due to emission correction that takes into account the systematic effects such as, for example, a template mismatch. Therefore, to test if our results are biased due to a poor emission subtraction, we re-calculate the ages and metallicities using 8 indices not affected by emission: G4300, Fe4383, $\mathrm{Ca} 4455, \mathrm{Fe} 4531, \mathrm{C} 4668, \mathrm{Mg} 1, \mathrm{Fe} 5270$, Fe5335. Basically we use all the indices except for the ones that may be affected by emission. It has been shown that even without Balmer lines, it is possible to obtain a reliable age (although with larger errors) (e.g. Chillingarian et al. 2008).

The differences between the mean gradients using these sets of indices and using the previous set of 4 indices are shown in Fig. 6. As can be seen from this figure, there is very good agreement between the results obtained using a different set of index combinations, giving confidence in the derived results. However, for the $[\mathrm{E} / \mathrm{Fe}]$ gradients there are three galaxies for which the values seem to differ slightly between the two methods.

Before continuing with the presentation of the results, we want to remind the reader that the stellar population parameters derived here are single stellar population (SSP)-equivalent parameters and can be interpreted as mean values weighted by the light of individual stars. In the case of the age, the mean values will be strongly biased towards the age of the youngest components.

\section{Results}

\subsection{Kinematics}

Figure 8 shows the line-of-sight position-velocity diagrams and velocity dispersion along the bar major-axis for the galaxies in our sample. Close examination of these diagrams can help to reveal the nature of the structures in the bar region. All the galaxies present disk-like structures from their kinematics. These structures are characterized by a central velocity dispersion minima ( $\sigma$ drops), central $\sigma$ plateaus and/or a clear rotating inner disks or ring as derived from the line-of-sight position-velocity diagrams. We define the central $\sigma$ as the maximum velocity dispersion in the bulge region. We have chosen this dispersion value to avoid being dominated in some galaxies by the low central velocity dispersion that could be indicative of nuclear star formation. Therefore, the maximum velocity dispersion should be closer to the velocity dispersion of the bulge.

Most of our galaxies show a "double-hump" rotation curve (Bureau \& Athanassoula 2005; Chung \& Bureau 2004), that is, the rotation curve first rises rapidly and reaches a local maximum, then drops slightly and then rises again slowly. These profiles were detected in $N$-body simulations of edge-on barred disks by Bureau \& Athanassoula (2005). They appear in strong bars and are detected at small viewing angles. The fact that these features are detected in pure $N$-body simulations indicate that they could arise naturally from the orbital structure of a barred disk and therefore gas dissipation is not indispensable. Central velocity dispersion minima occur in $70 \%$ of our sample. Galaxies not showing a $\sigma$-drop are: NGC 2859, NGC 2935 , NGC 2950, NGC 2681, NGC 2217, NGC 1169 and NGC 1358. However, the NGC 2950, NGC 2217 and NGC 2859 shallow and wide central dip could be also considered as a $\sigma$-drop. Chung $\&$ Bureau (2004) found $\sigma$-drops in $40 \%$ of their barred sample, while Peletier et al. (2007) found it in $50 \%$ of their sample of 24 barred and unbarred galaxies. Márquez et al. (2003) reported that 12 out of 14 of the analyzed galaxies with $\sigma$ drops showed an inner disk from their HST images. The standard explanation for these drops is the presence of cold central 

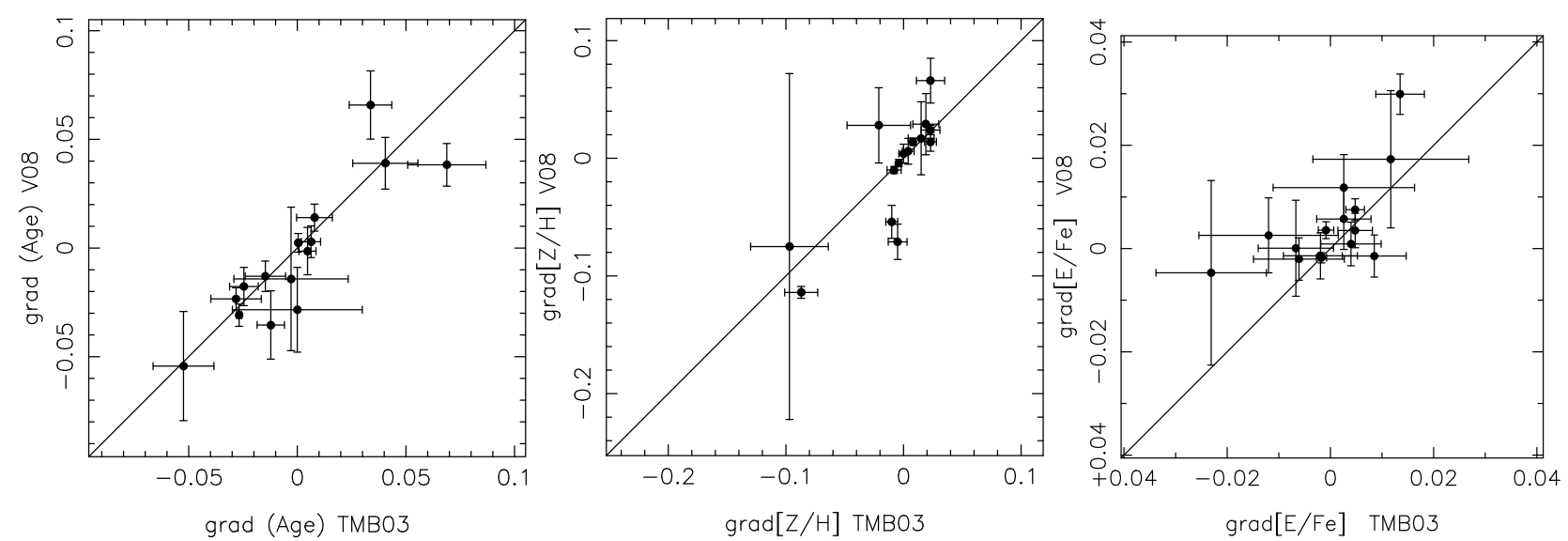

Fig. 5. Comparison between the mean gradients measured using TMB03 and V08 models. We have carried out a comparison of the metallicity and age gradients using two different models to asses the dependance of the results on the model choice. As it can be seen, the comparison is very good in general.
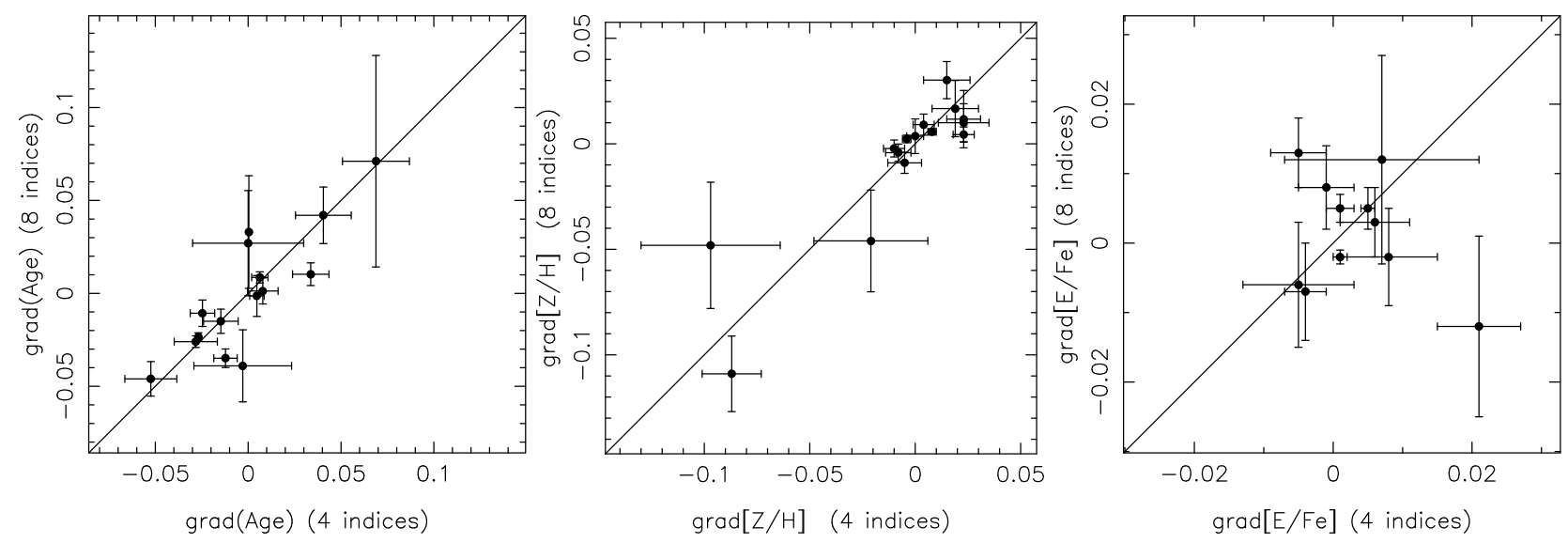

Fig. 6. Comparison between the age, metallicity gradients obtained using 4 indices or a combination of 8 indices not affected by emission (see text for details). There is very good agreement between the results obtained using different sets of index combination.

stellar disks originating from gas inflow (Emsellem et al. 2001; Wozniak et al. 2003). Wozniak \& Champavert (2006) predicted from dynamical simulations of drops in barred galaxies that these features can last for more than $1 \mathrm{Gyr}$ if the central region is continuously fed by fresh gas leading to a continuous star formation activity. In their simulations, continuous star formation is needed to replace the heated particles by new low- $\sigma$ ones. They predict that as the star formation disappears, the drop will disappear as well. However, this disappearance is not instantaneous; they find that a $\sigma$-drop disappears at a linear rate of $10 \mathrm{~km} \mathrm{~s}^{-1}$ in $500 \mathrm{Myr}$, when star formation is switched off. On the contrary, Athanassoula \& Misiriotis (2002) found these features in $N$-body dissipationless simulations, and Bureau \& Athanassoula (2005) showed that they can arise from the orbital structure of strongly barred galaxies. These authors noted that, in their simulations of strongly barred galaxies, the width of the central flat section of the velocity dispersion profiles was roughly the same as that of the rapidly rising part of the rotation curve, and suggest a common origin ( $x 1$ orbits). However, these $N$-body simulations have no stellar population predictions and, therefore, it is difficult to compare them to our observations. These two possible $\sigma$-drop origins (cold dissipative disk vs. bar orbital stellar structure) can be separated by studying the age in the central parts of the galaxies with $\sigma$-drops. If the $\sigma$-drop has to be maintained by continuous gas replenishment and star formation, we would expect a very young population to dominate the central regions. On the other hand, if the drops are due to an old stellar structure, we would expect the central parts to be dominated by an old stellar component. We will present the stellar population of the central parts of these galaxies in the second paper of this series, but we can already advance that, while most of these galaxies indeed have young components and star formation in their centers, eight galaxies (NGC 1358, NGC 2523, NGC 2859, NGC 2962, NGC 4245, NGC 4314, NGC 4643 and NGC 5101) have old central luminosity-weighted ages ( $>3 \mathrm{Gyr}$ ) despite having central dips or plateaus. However, this fact does not necessarily imply that the ages of the cold component are old, since we are measuring luminosity weighted ages and a bulge component could be contributing to the derived old age. It would be necessary to disentangle the populations coming from both components. This will be done in detail in a forthcoming paper addressing the central regions of barred galaxies.

Eight out of the 20 galaxies are classified as double barred (Erwin 2004) NGC 1433, NGC 2217, NGC 2681, NGC 2859, NGC 2950, NGC 2962, NGC 3081, NGC 4314. They do not show any difference in their kinematic profiles, although all of them show a plateau in their central stellar velocity dispersion.

\subsection{Mean stellar population parameters in the bar region}

In order to relate the bar properties to their stellar population parameters, we first calculate the mean stellar age, $[\mathrm{E} / \mathrm{Fe}]$, and 
metallicity of the bar by averaging the derived values along the bar and weighting by the errors in these parameters. We would like to clarify the difference between what we mean by the age of the bar and the age of the bar stellar population; the latter is the mean age of the stars as derived from the stellar populations, and the age of the bar is, for us, the age constraint to the time of bar formation that we obtain from the stellar population analysis, mean values and gradients of the ages and metallicities. The mean values are reported in Table 4 . The first thing that can be seen is that our sample of bars exhibits a large dispersion in the mean ages of their stellar populations. The differences in the mean ages in the bars between the different galaxies can be as large as $10 \mathrm{Gyr}$, with some galaxies showing a mean bar age older than $10 \mathrm{Gyr}$ and others younger than $<1$ Gyr. Figure 9 shows the relationship between the mean stellar age, the mean $\mathrm{Z}$ and the average $[\mathrm{E} / \mathrm{Fe}]$ in the region of the bar against the maximum central velocity dispersion. As can be seen, there is a trend in which galaxies with a smaller central velocity dispersion are also the ones showing younger mean ages. In the same way, galaxies with lower maximum central velocity dispersion tend to have lower values of $[\mathrm{E} / \mathrm{Fe}]$ and metallicity. In this figure, we have separated the galaxies hosting a nuclear AGN from those without; there is no significant difference in the distribution of the two samples.

This is the first time that stellar population parameters are derived for the bar region in galaxies. It is interesting to notice that the trends shown in Fig. 9 are similar to those found for galaxy bulges (Proctor \& Sansom 2002; Morthy \& Holtzman 2006) with values of the metallicity and $[\mathrm{E} / \mathrm{Fe}]$ slightly lower than those found in the bulges. This result points out an intimate link between the evolution of the bar and the bulge.

Gadotti \& de Souza (2006), using color as a proxy for ages, also found a large difference in the ages of the stars in a sample of 18 barred galaxies. These authors found a relation between the color of the bar and the morphological type of spiral, it being more likely that a later type spiral hosts a younger bar. We did not find any correlation between the mean age of the stars in the bars and the morphological type of the galaxies, although we do not have late spirals in our sample.

These authors also found a weak correlation between the mean age of the bar and the length of the bar, with older bars also being longer. We did not find any correlation between these two parameters.

As a cautionary note, it is not a good assumption to consider broad-band colors as a probe for age, assuming a constant metallicity for all the galaxies, as we have shown here that bars in different galaxies show a wide metallicity distribution.

\subsection{Stellar populations along the bar}

As shown in Sect. 4.4, we fit a slope to the derived age, metallicity and $[\mathrm{E} / \mathrm{Fe}]$ distributions with a simple linear regression weighted by the errors in the $y$-direction (Table 5). We find that most galaxies show a gradient different from zero in both age and metallicity. Figure 7 shows the indices at the beginning and end of the bar (Sect. 4.3) together with the predictions of the models by V08. The galaxies with the lowest mean metallicity values, systematically, have younger and more metal poor populations at the end of the bar (these are the data points on the left-hand side of the grid in Fig. 7). The more metal rich bars (data points on the right-hand side of the grid in Fig. 7) show more complex behavior with most of the galaxies showing younger and more metal rich populations at the end of the bar.
Table 4. Mean stellar population parameters. Mean ages (2), mean metallicities (3) and [E/Fe] (4) (see Sect. 4.4 for an explanation on the parameter derivation).

\begin{tabular}{lccc}
\hline \hline Galaxy name & $\langle$ log age $\rangle$ & $\langle[\mathrm{Z} / \mathrm{H}]\rangle$ & $\langle[\mathrm{E} / \mathrm{Fe}]\rangle$ \\
\hline NGC 1169 & $1.172 \pm 0.038$ & $0.069 \pm 0.032$ & $0.112 \pm 0.029$ \\
NGC 1358 & $1.043 \pm 0.048$ & $0.000 \pm 0.034$ & $0.225 \pm 0.029$ \\
NGC 1433 & $0.266 \pm 0.031$ & $0.005 \pm 0.041$ & $0.138 \pm 0.038$ \\
NGC 1530 & $0.078 \pm 0.027$ & $-0.023 \pm 0.050$ & $0.267 \pm 0.073$ \\
NGC 1832 & $0.153 \pm 0.015$ & $-0.184 \pm 0.028$ & $0.002 \pm 0.024$ \\
NGC 2217 & $0.344 \pm 0.045$ & $0.644 \pm 0.046$ & $0.336 \pm 0.030$ \\
NGC 2273 & $0.241 \pm 0.020$ & $0.064 \pm 0.030$ & $0.111 \pm 0.025$ \\
NGC 2523 & $0.530 \pm 0.062$ & $0.313 \pm 0.054$ & $0.136 \pm 0.031$ \\
NGC 2665 & $0.334 \pm 0.076$ & $-0.606 \pm 0.092$ & $-0.010 \pm 0.076$ \\
NGC 2681 & $0.439 \pm 0.029$ & $-0.602 \pm 0.039$ & $0.015 \pm 0.036$ \\
NGC 2859 & $1.022 \pm 0.029$ & $-0.064 \pm 0.021$ & $0.238 \pm 0.021$ \\
NGC 2935 & $0.374 \pm 0.056$ & $-0.192 \pm 0.059$ & $0.114 \pm 0.052$ \\
NGC 2950 & $1.053 \pm 0.025$ & $-0.311 \pm 0.019$ & $0.114 \pm 0.052$ \\
NGC 2962 & $0.920 \pm 0.035$ & $0.029 \pm 0.026$ & $0.135 \pm 0.022$ \\
NGC 3081 & $0.950 \pm 0.157$ & $-0.225 \pm 0.124$ & $-0.060 \pm 0.096$ \\
NGC 4245 & $0.774 \pm 0.045$ & $-0.095 \pm 0.031$ & $0.085 \pm 0.028$ \\
NGC 4314 & $0.297 \pm 0.031$ & $0.066 \pm 0.038$ & $0.105 \pm 0.032$ \\
NGC 4394 & $0.956 \pm 0.033$ & $-0.202 \pm 0.024$ & $0.131 \pm 0.025$ \\
NGC 4643 & $0.741 \pm 0.013$ & $0.321 \pm 0.009$ & $0.126 \pm 0.006$ \\
NGC 5101 & $0.477 \pm 0.021$ & $0.182 \pm 0.213$ & $0.113 \pm 0.015$ \\
\hline
\end{tabular}

Table 5. Metallicity and age gradients.

\begin{tabular}{lrr}
\hline \hline Galaxy name & $\mathrm{d}[\mathrm{Z} / \mathrm{H}] / \mathrm{d} r$ & $\mathrm{~d} \log ($ age $) / \mathrm{d} r$ \\
\hline NGC 1169 & $0.023 \pm 0.008$ & $0.0004 \pm 0.0006$ \\
NGC 1358 & $0.015 \pm 0.011$ & $-0.0029 \pm 0.0263$ \\
NGC 1433 & $0.004 \pm 0.005$ & $-0.0147 \pm 0.0093$ \\
NGC 1530 & $0.052 \pm 0.012$ & $-0.0143 \pm 0.0070$ \\
NGC 1832 & $-0.005 \pm 0.008$ & $-0.0282 \pm 0.0116$ \\
NGC 2217 & $0.023 \pm 0.012$ & $-0.0524 \pm 0.0140$ \\
NGC 2273 & $0.019 \pm 0.011$ & $-0.0122 \pm 0.0063$ \\
NGC 2523 & $-0.021 \pm 0.027$ & $0.0000 \pm 0.0299$ \\
NGC 2665 & $-0.087 \pm 0.014$ & $0.0405 \pm 0.0150$ \\
NGC 2681 & $-0.097 \pm 0.033$ & $0.0688 \pm 0.0180$ \\
NGC 2859 & $-0.008 \pm 0.006$ & $0.0079 \pm 0.0082$ \\
NGC 2935 & $0.064 \pm 0.040$ & $-0.1371 \pm 0.0300$ \\
NGC 2950 & $-0.000 \pm 0.004$ & $0.0047 \pm 0.0039$ \\
NGC 2962 & $0.021 \pm 0.015$ & $-0.0257 \pm 0.0138$ \\
NGC 3081 & $-0.587 \pm 0.556$ & $-0.0661 \pm 0.6848$ \\
NGC 4245 & $-0.010 \pm 0.005$ & $0.0337 \pm 0.0098$ \\
NGC 4314 & $-0.014 \pm 0.005$ & $0.0184 \pm 0.0096$ \\
NGC 4394 & $0.023 \pm 0.005$ & $-0.0246 \pm 0.0066$ \\
NGC 4643 & $-0.004 \pm 0.002$ & $0.0063 \pm 0.0044$ \\
NGC 5101 & $0.008 \pm 0.002$ & $-0.0268 \pm 0.0012$ \\
\hline
\end{tabular}

Figure 10 shows the age and metallicity distribution profiles for the galaxies in our sample. We have not included in this analysis NGC 1530, NGC 3081, NGC 4314 and NGC 2935 due to the large fitting errors. Seven galaxies have metallicity gradients significantly (more than 3- $\sigma$ significance) different from zero: NGC 1169, NGC 2217, NGC 4394, and NGC 5101 (positive) NGC 2665, NGC 2681, NGC 4245 (negative).

We therefore confirm the results from Pérez et al. (2007), where we found, in a much more reduced sample, that some of the bars had positive metallicity gradients and negative age gradients. These negative age gradients are expected since, as it 

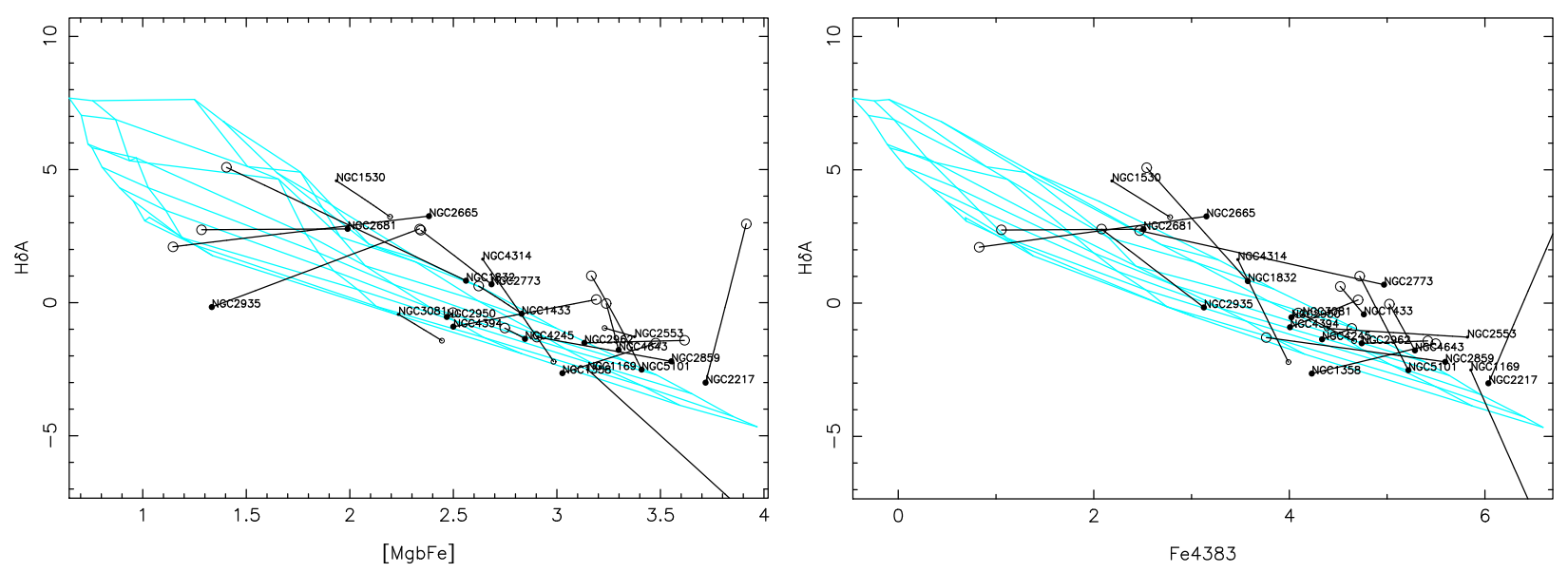

Fig. 7. $[\mathrm{MgFe}]$ and $\mathrm{Fe} 4383$ indices vs. $\mathrm{H} \delta_{\mathrm{A}}$ measured in the inner (filled symbols) and outer (open symbols) parts of the bar. Overplotted are the models by Vazdekis. Models are shown with ages = 1, 1.41, 2.00, 2.82, 3.98, 5.62, 7.98, 11.22, 15.85 (Gyr) from top to bottom, and metallicities, $[\mathrm{Z} / \mathrm{H}]=-1.68,-1.28,-0.68,-0.38,0.0,0.2$ from left to right.

has been shown in numerical simulations, there is an accumulation of young stellar populations trapped in elliptical-like orbits along the bar, near the ultra-harmonic resonance (Wozniak 2007). Although expected, this negative age gradient should be further investigated with models to check whether the gradient can remain for longer than $3 \mathrm{Gyr}$, the time at which the simulations were stopped and that is shorter than the age of the last star formation bursts found for our galaxies.

What we found more striking is the presence of a positive metallicity gradient in four galaxies, i.e. the younger stars at the end of the bar are also more metal rich. These gradients are difficult to understand in the context of our current understanding of bar formation (see Sect. 6), if they are a consequence of the star formation that ocurred during its formation, this would imply that there has been chemical enrichment and, therefore, that the star formation lasted for a long period of time or that it has been very efficient.

The galaxies showing a negative metallicity gradient (i.e. less metal rich in the outer parts of the bar) might be reflecting the original disk gradient. Only for a few galaxies have the disk radial stellar gradients been derived. Mollá et al. (1999) derived shallow negative gradients for the $\mathrm{Mg}_{2}$ and $\mathrm{Fe} 5270$ indices in the discs of NGC 4303 (SABbc), NGC 4321 (SABbc) and NGC 4535 (SABc). Ryder et al. (2005) obtained the radial disk profiles of the $\mathrm{Mg}_{2}$ and Fe5270 indices for a sample of 8 latetype galaxies with similar results. In a recent work, Yoachim \& Dalcanton (2008) have derived luminosity weighted ages and metallicities in the disk region of nine edge-on galaxies, finding radial negative age gradients and almost no metallicity gradient. In order to follow up this issue, we have collected deep spectroscopic information on the disk of our sample (Sánchez-Blázquez et al. 2009, in preparation).

Figure 11 shows the relation between the metallicity gradient along the bar and the velocity dispersion. It can be seen that the galaxies with velocity dispersions higher than $170 \mathrm{~km} \mathrm{~s}^{-1}$ tend to have positive metallicity gradients, while the lower velocity dispersion galaxies show a larger dispersion in their metallicity gradient value. Similar behavior is found for the age gradient, where galaxies with large velocity dispersion tend to have a shallow positive age gradient while for the lower velocity dispersion galaxies the absolute value of the age gradient is larger, with both negative and positive gradients. This seems to imply that the stellar population gradient is related to the mass of the galaxy's bulge. To test whether there is a link between the mass of the bulge and the total galaxy mass, we have investigated the correlation between the bulge dispersion velocity and the total $H$-band luminosity (as a tracer of the stellar galaxy mass). There is, indeed, a correlation between the maximum central velocity dispersion and the total $H$-band luminosity, which might link the observed metallicity gradients to the stellar mass of the galaxy. Further investigation needs to be done to address this issue. The galaxies with negative metallicity gradients systematically have positive age gradients and have average bar-age values of the order of 2 Gyr (except for NGC 4245 with an average of 5 Gyr).

\subsection{Correlation with bar/galaxy parameters}

We want to check possible connexions between observational structural bar parameters that are thought to be important in the bar formation and evolution with the stellar population parameters derived in this work. The bar strength is determinant in the orbital structure of bars and it is thought to evolve as the bar ages (e.g. Athanassoula 2005). For this purpose, we have used the available bar strengths obtained as the ratio of the tangential force to the mean radial force derived from the NIR images (Combes \& Sanders 1981; Block et al. 2001, 2004). For 13 out of the 20 galaxies there are values for the bar-strength from the literature (Table 1). No clear relation is found between the barstrength and the metallicity gradients.

It has been suggested that older bars should become longer and thinner in time as their pattern speed decreases. To check if there is any systematic behavior between the bar sizes and the metallicity gradients, we have checked the absolute bar sizes and the relative sizes of the bar with respect to their disk for the galaxies in our sample. We find, in agreement with previous studies (Erwin 2005), that the bar sizes are amongst the typical sizes of S0-Sab (between 1 and $8 \mathrm{kpc}$ in our sample) and that the sizes correlate well with the disk size (Pérez et al. 2005; Erwin 2005). There is no significant trend between the size of the bar and the metallicity and age gradient. However, there might be a hint of correlation pointing to larger bars having positive age gradients, negative metallicity gradients and an average young population. But, due to low number statistics and the large spread in the values, nothing conclusive can be said in this respect. However, it would be interesting to follow up this point, calculating the bar-size for all the galaxies with new broad-band data 

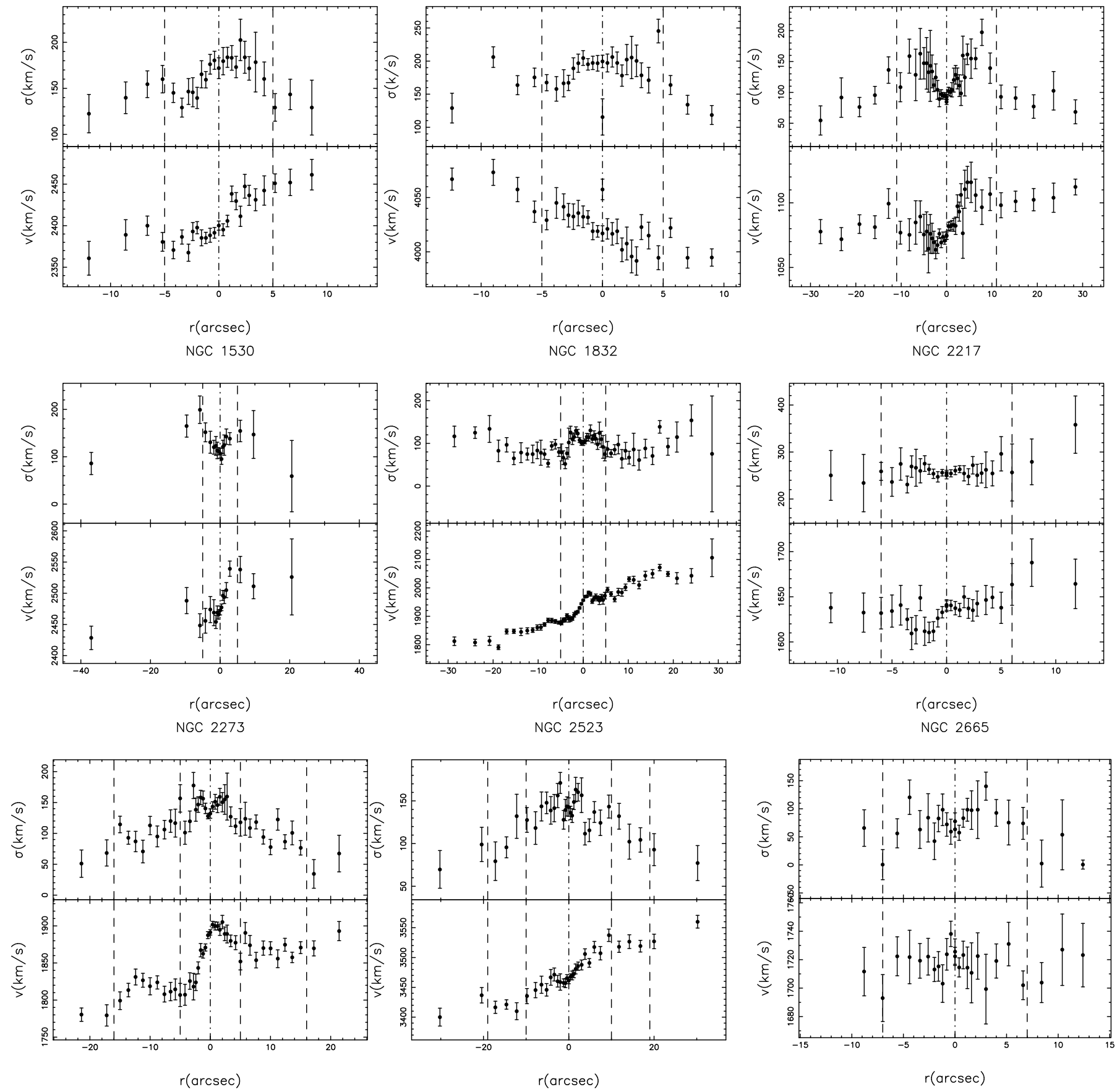

Fig. 8. Position-velocity diagrams and velocity dispersion profiles along the bar major-axis for our sample of galaxies. Dashed lines indicate the beginning of the region dominated by the bar.

to test if there is a significant trend of the bar stellar population parameters with bar-size. Rings are located near resonances induced by the bar component; from numerical simulations outer rings form in time-scales longer than $1 \mathrm{Gyr}$ and therefore, they have been associated to a relatively aged system. However, in some simulations, rings appear very quickly, about $0.2 \mathrm{Gyr}$ after the bar formation (Rautiainen \& Salo 2000). We do not see any correlation between the presence of an outer ring and the bar age. Some of the bars with young populations and negative metallicity gradients present outer rings, therefore, the presence or absence of an outer ring is not a good discriminator of the bar age.
There seems to be no clear correlation with morphological type, although we do not cover a large range in morphological types $(\mathrm{S} 0-\mathrm{Sbc})$, the galaxies with positive metallicity gradients in the bar region are not particularly different in morphology to the rest of the sample.

\subsubsection{AGN connection}

Since the bar is an efficient mechanism to drive material to the central parts of the galaxy its role in feeding the central massive black hole and the bar-AGN connection has been very much discussed. Recent statistical studies (e.g. Knapen 2004) have 
NGC 268
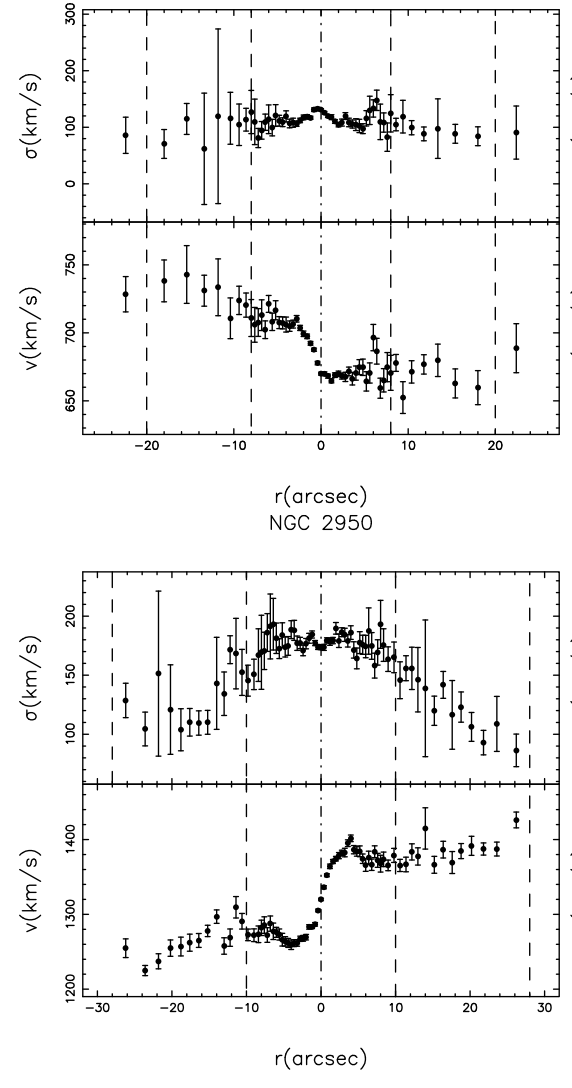

NGC 4245

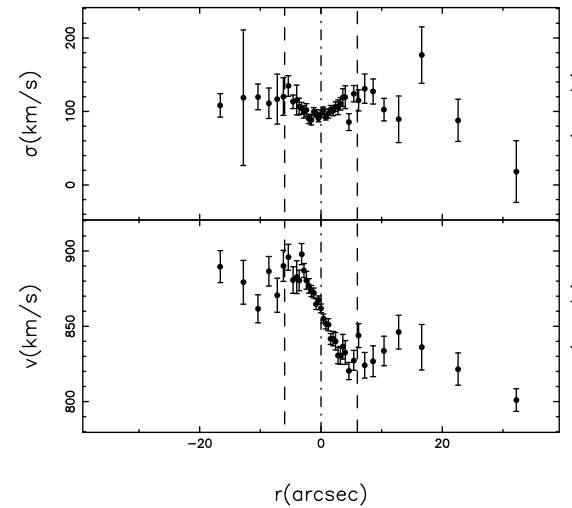

NGC 4643

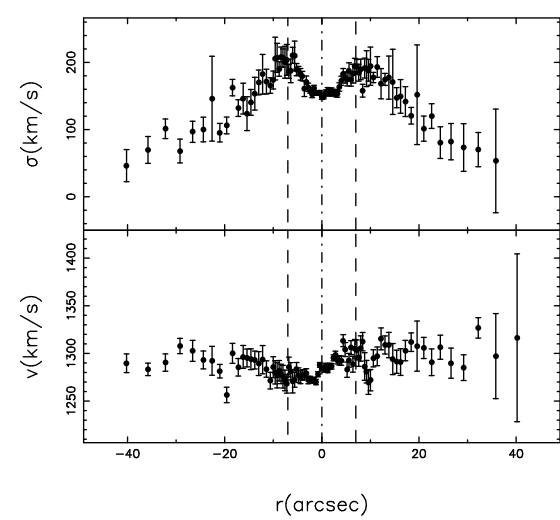

NGC 2859
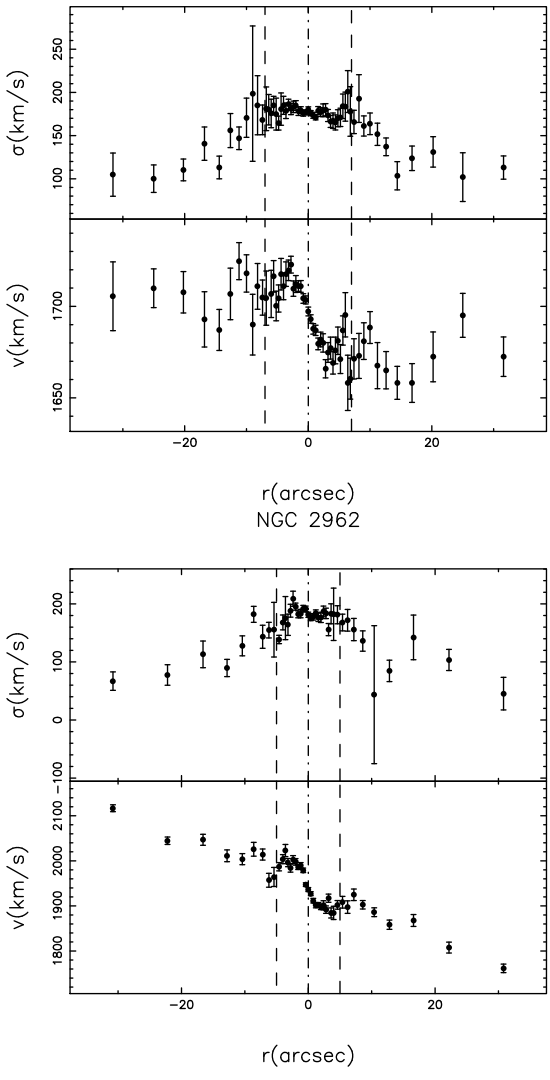

NGC 4314

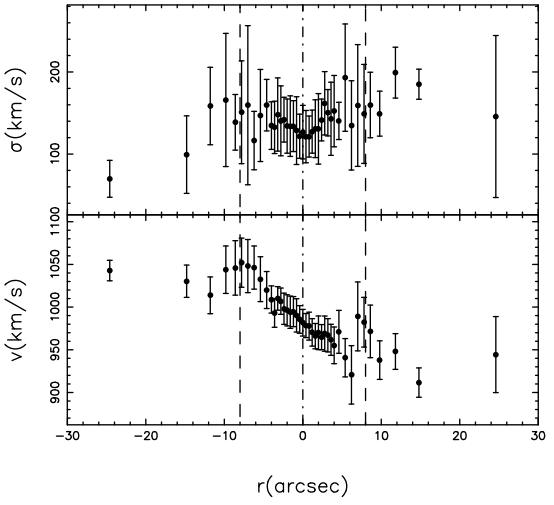

NGC 5101

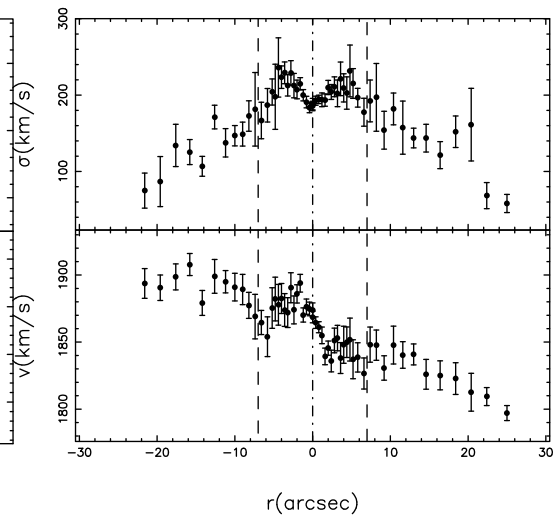

NGC 2935
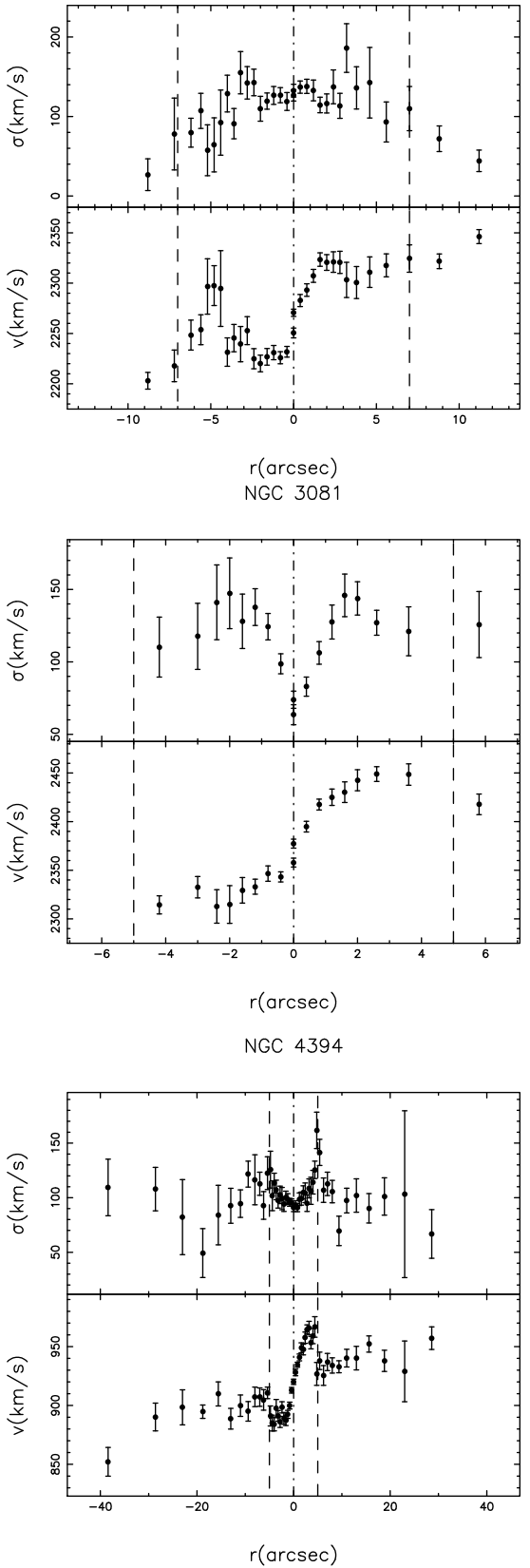

Fig. 8. continued. 

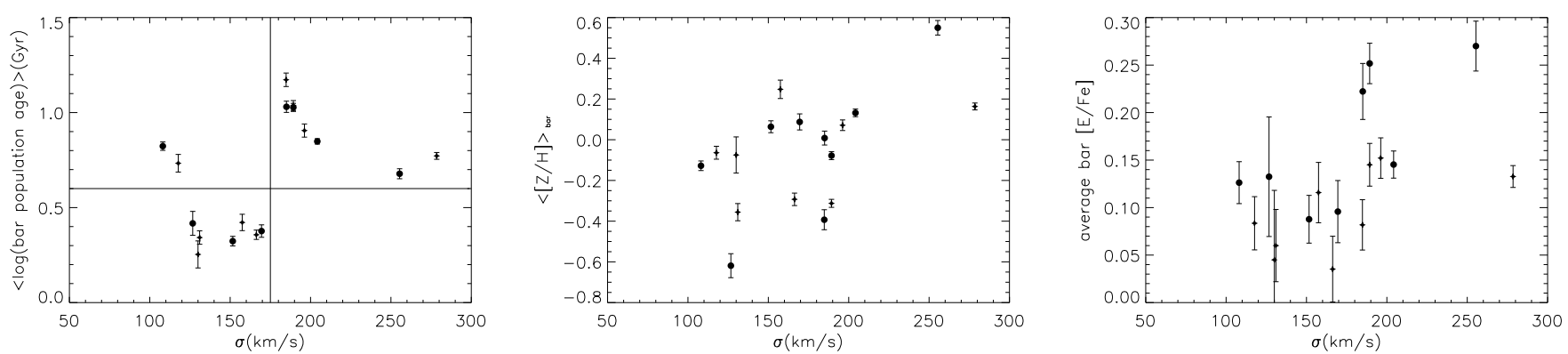

Fig. 9. Mean age, metallicity and $[\mathrm{E} / \mathrm{Fe}]$ in the bar region against maximum central velocity dispersion. The diamonds correspond to the galaxies classified as hosting and AGN, the circles are the galaxies with non-active nuclei. Notice that galaxies with lower maximum central velocity dispersions are also the ones with younger ages and lower $\mathrm{Z}$ and $[\mathrm{E} / \mathrm{Fe}]$ values; these trends are similar to those found for the bulge region.
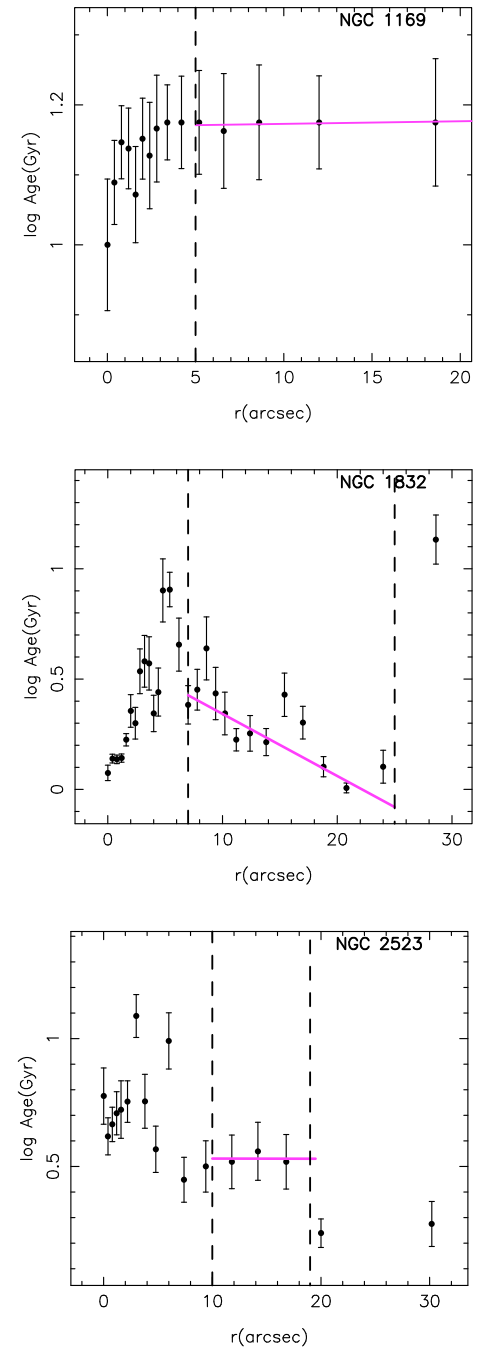
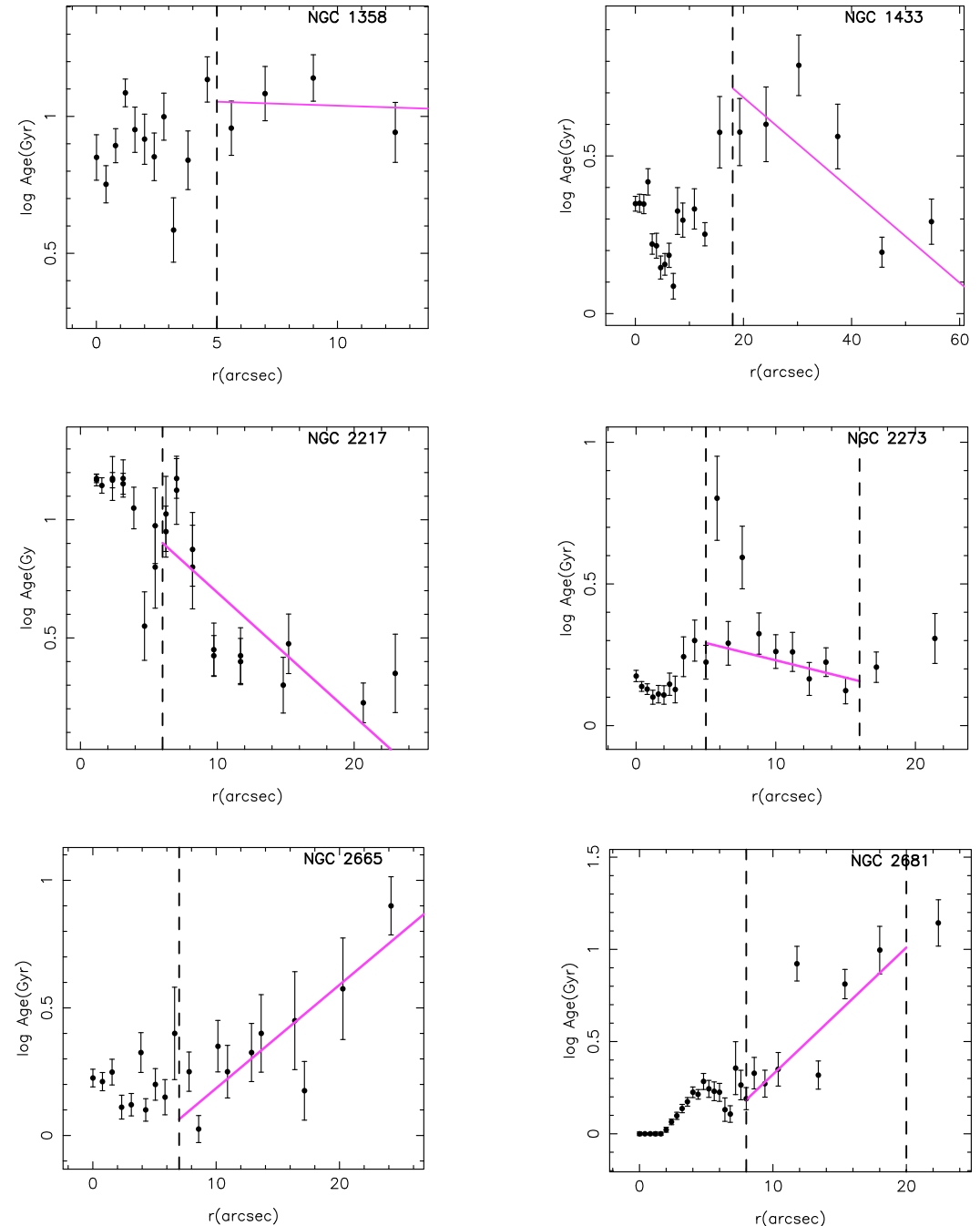

Fig. 10. SSP-equivalent age and metallicity along the radius. Dashed lines indicate the beginning and the end of the bar region. A linear fit to the bar region is also plotted.

concluded that there is not a statistically significant relation between the presence of an AGN in barred galaxies. Part of the problem regarding the lack of correlation between the AGN and the bar presence could be the different time-scales of the different processes. There have been recent claims, using broad-band photometry (Gadotti \& Souza 2004), that active galactic nuclei tend to have young bars, while galaxies with old bars do not generally host AGNs. These studies are based on the assumption that the change in broad-band galaxy color is due to age and not to metallicity as well. Because spectra provide a better way to discern between age and metallicity, and since the indices are not affected by dust, we have carried out this project also to see whether there is any correlation between the bar ages and metallicities with the presence of an AGN. In Fig. 9 we have 

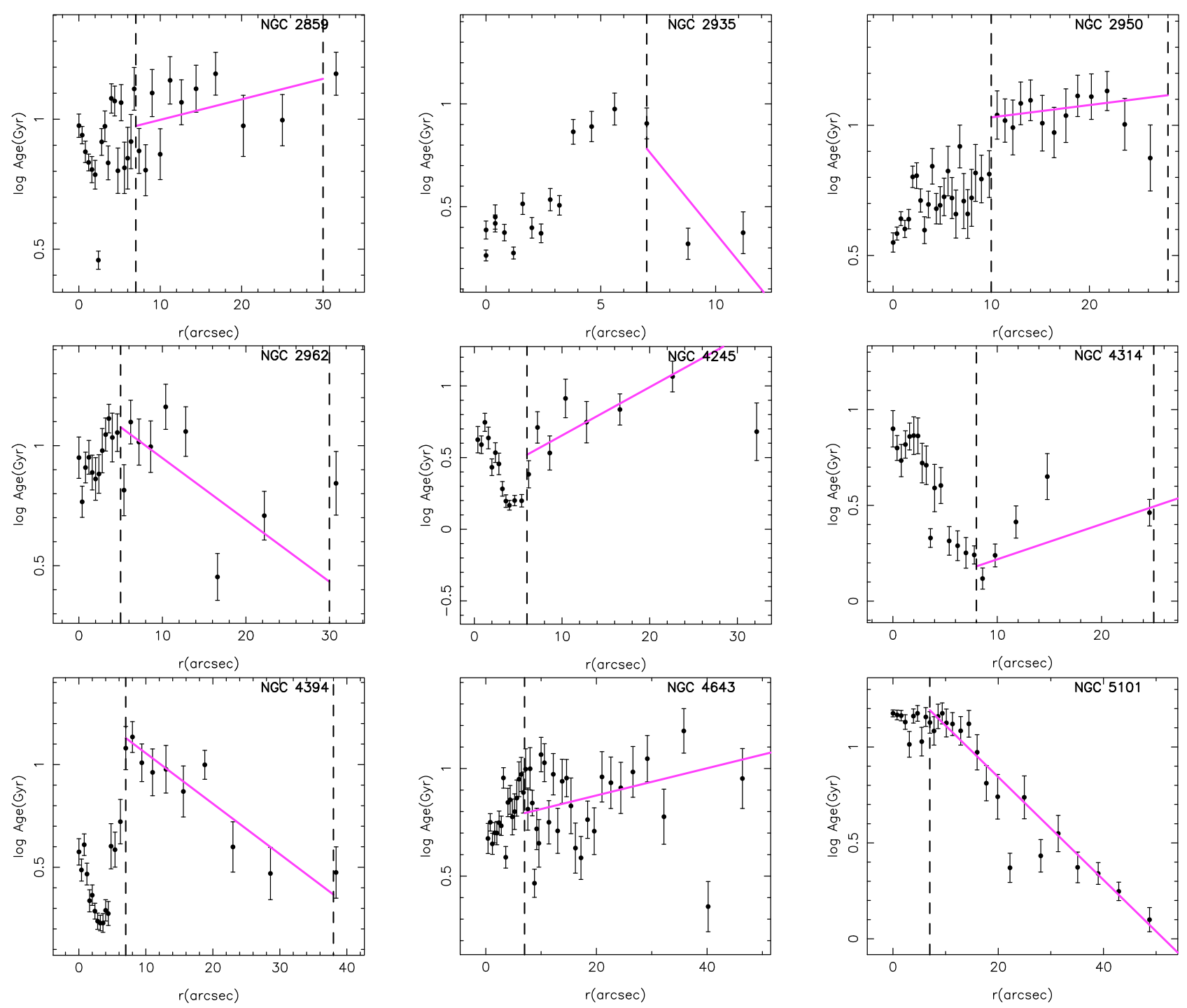

Fig. 10. continued.

separated, with different symbols, galaxies hosting an AGN. We do not see any significant correlation with mean bar age and metallicity nor with the derived gradients. This might only reflect the small number statistics and a larger sample would be needed to properly cover the parameter space. Therefore, we do not reproduce the results of Gadotti \& de Souza (2006). However, the correlation that these authors found is weak and they were using colors as a proxy for age.

\section{Discussion and summary}

We have derived the kinematics and the distribution of ages and metallicities along the bar in a sample of 20 barred galaxies to study the question of whether bars are long-lived or whether they are destroyed and reformed in a few Gyr. We also want to explore the relation between the age, and stellar populations of the bar and other global physical properties of the host galaxies.

From the study of the kinematics along the bar, we have found that all the galaxies in the sample show a disk-like component in their centers, appearing as dips or plateaus in their central stellar velocity dispersion and as stellar rotating disks. Therefore, this implies that bars, as expected, have a strong influence on the building up and later evolution of the central component. This aspect will be studied in more detail in Sánchez-Blázquez et al. (in prep) where we compare the bulge ages and metallicities of barred and unbarred bulges of otherwise similar galaxies.

We have derived ages and metallicities from Lick/IDS linestrength indices of single stellar populations (SSP). It is clear that the true star formation history in these objects will be more complicated than a simple SSP. However, a lot of information can be derived from the SSP analysis. The SSP-equivalent age of a composite stellar population (CSP) is dominated primarily by the age of the youngest component and the mass fraction of the different populations. The SSP-equivalent metallicity reflects a $V$-band luminosity weighted chemical composition (Serra \& Trager 2007).

As bars form from stars already in the disc, we have to distinguish between stellar population gradients that may have already been present when the bar formed and those that formed during the bar formation or afterwards. There are not many studies analysing the stellar population of discs outside the MW. In particular, for early-type spirals, only broadband colours traditionally have been used to estimate the ages and metallicities 

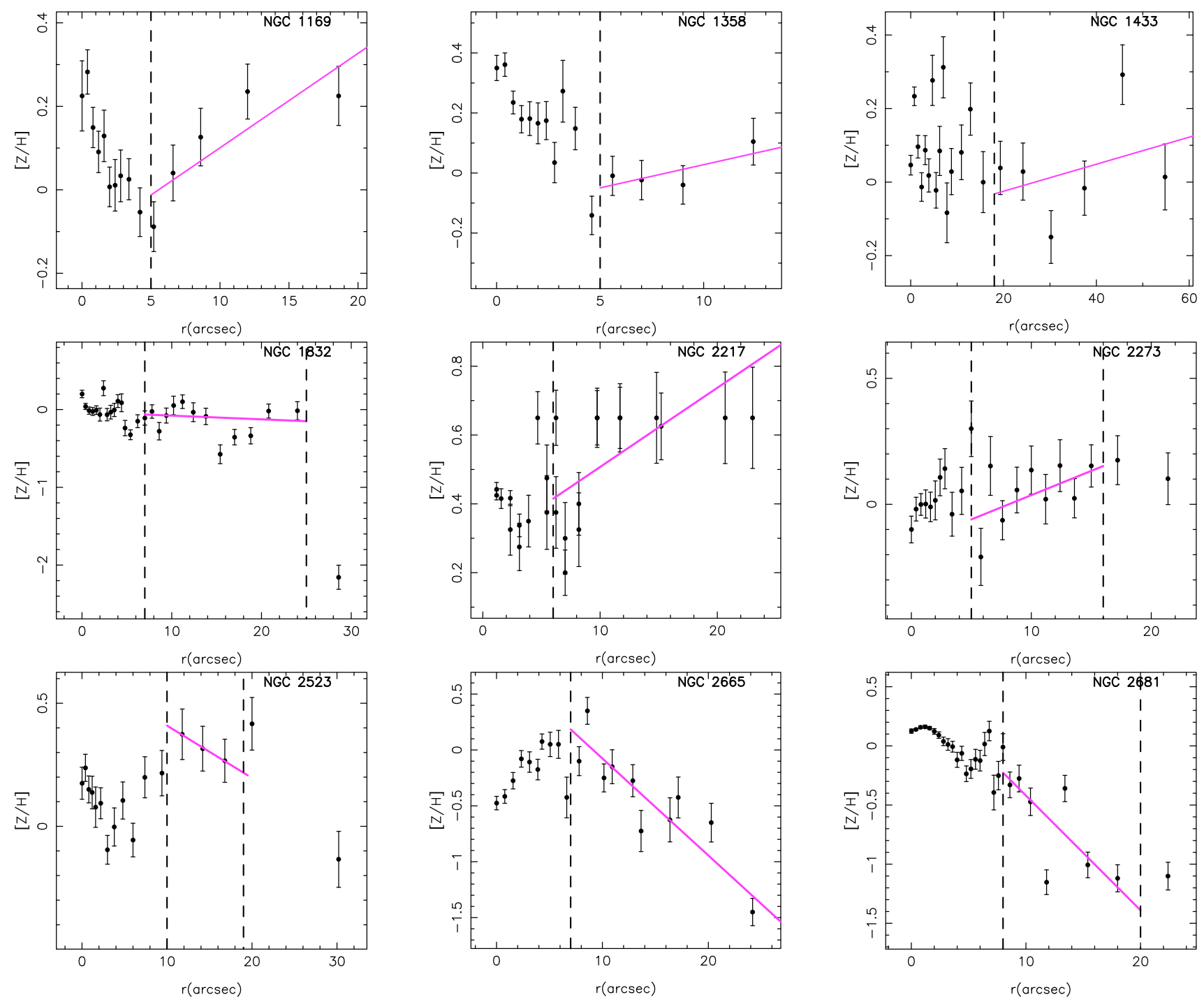

Fig. 10. continued.

of the stellar discs. However, stellar parameters are very difficult to obtain with broad band colours. Yoachim \& Dalcanton (2008) measured Lick indices in the thin and thick disc of edgeon galaxies. They found a very strong age gradient and a null or negative metallicity gradient dependending on the index they were using. In the MW a negative age and metallicity gradient are measured. We will assume, for our discussion, that this is the general trend in discs before the formation of the bar.

We have found three different types of bars according to their metallicity and age distribution along the bar:

1) Bar with negative metallicity gradient. These bars show a mean young/intermediate population $(<2 \mathrm{Gyr})$ and have amongst the lowest central stellar velocity dispersion of the sample. They also tend to have positive age gradients (i.e. younger populations at the beginning of the bar). In fact, all the galaxies showing these trends posses circumnuclear regions with a young population $(1.5 \mathrm{Gyr})$. The negative gradient can be understood as the original gradient of the disc. This is because a small fraction of young stars can bias the meanage measured with SSP towards very small values. However, the mean metallicity is more sensitive to the more massive component. In this picture, the circumnuclear region would strongly affect the mean age at the beggining of the bar (lowering it) but its influence on the mean metallicity would not be as strong. Since one would expect the original metallicity gradients to flatten by the velocity dispersion of the bar (Friedli et al. 1994), the presence of metallicity gradients may indicate that they formed recently.

2) Bars with null metallicity gradients. The galaxies that do not show any gradient in their metallicity distribution along the bar have negative age gradients, that is, their ends of the bar are younger. These galaxies present gradients in both metallicity and age compatible with the models that studied mixing of metals and the distribution of stellar population ages in bars using numerical simulations (e.g. Wozniak 2007; Friedli et al. 1994). They predict low age regions at the end of bars due to the accumulation of young stellar populations trapped in orbits near the ultra-harmonic resonance. In the simulations, most of the star formation happens during the first Gyr. We observe that although the population at the end of the bar is younger than the average bar value, it is still larger than 3 Gyr. This fact might indicate that the bar in these galaxies is at least 3 Gyr old. 

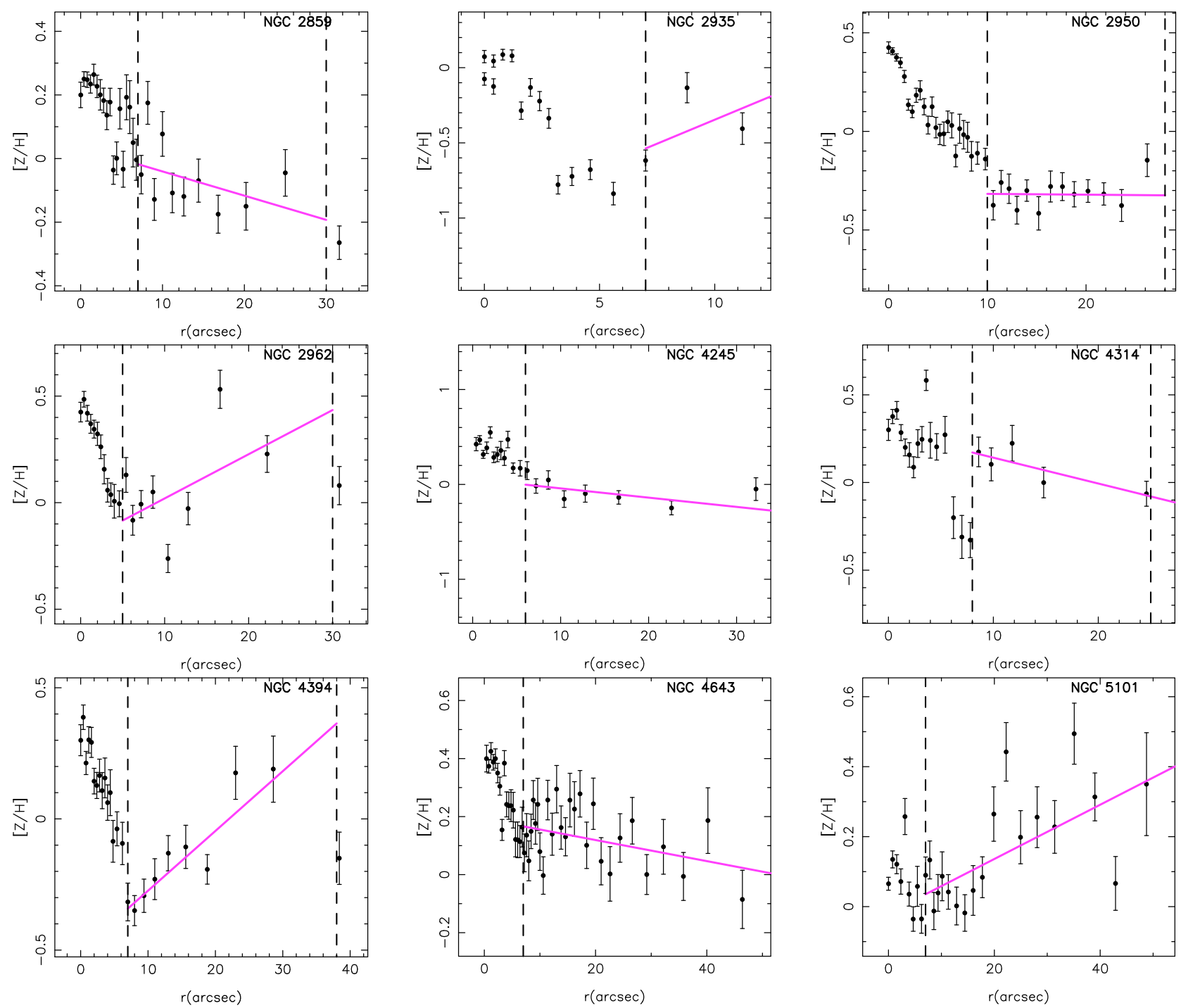

Fig. 10. continued.

3) Bars with positive metallicity gradients. These galaxies have older mean ages in their stellar populations. They are also the ones with more massive bulges (larger $\sigma$ ). In principle, these positive metallicity gradients are difficult to understand in light of our current knowledge of the formation and evolution of bars. Hydrodynamical simulations indicate that, even if a metal rich population can be formed at the end of the bar, the diffusion timescale in this region is very short, of the order of 50 Myr (see Fig. 3 in Wozniak 2007). The metallicity gradient in disc galaxies is believed to be negative (see references above) and, therefore, it is not likely that this gradient is a relic of the original metallicity gradient in the disc. We do not have a definitive explanation of the origin of these metallicity gradients. One posibility is the presence of an inner ring at the beginning of the bar with enriched SF that could be populating the extremes of the bar in the same fashion as we explained for the presence of young stellar populations in the outer parts of the bar (case 2). However, we do not see this effect in all the galaxies hosting a nuclear young ring. Furthermore, the luminosity weighted mean age of the stars at the beggining of the bar is older than that in the external parts which is not expected if the bar ends are being populated with an enriched population coming from the inner ring. A projection effect of the old metal-rich population of the bulge on the bar region is not a likely scenario since, as it can be seen from the kinematics, the outer part of the bars are dominated by a cooler stellar component.

It has been claimed that the star formation in bars follows a sequential pattern, where younger bars $(<1 \mathrm{Gyr})$ would show star formation along the entire bar and later the star formation would be concentrated at the bar-ends and the nuclear region (Phillips 1993; Martin \& Friedli 1997; Martin \& Roy 1995; Friedli \& Benz 1995; Verley et al. 2007). We have checked the galaxies for emission lines coming from HII regions to see if the ones showing negative metallicity gradients systematically present HII along their bars.

NGC 2665 and NGC 1530 show nebular emission along their bars belonging to HII regions, which reflects very recent star formation events. Unfortunately, we do not have high $\mathrm{S} / \mathrm{N}$ information on the stellar indices for the whole bar in NGC 1530 that would allow us to establish the stellar population age and metallicity. NGC 2665, on the other hand, is one of the galaxies that show a negative metallicity and a positive age gradient along its bar. This is compatible with the idea of this sequential star formation pattern if we assume that these bars formed less than 3 Gy ago. However, other bars such as NGC 4245 and 


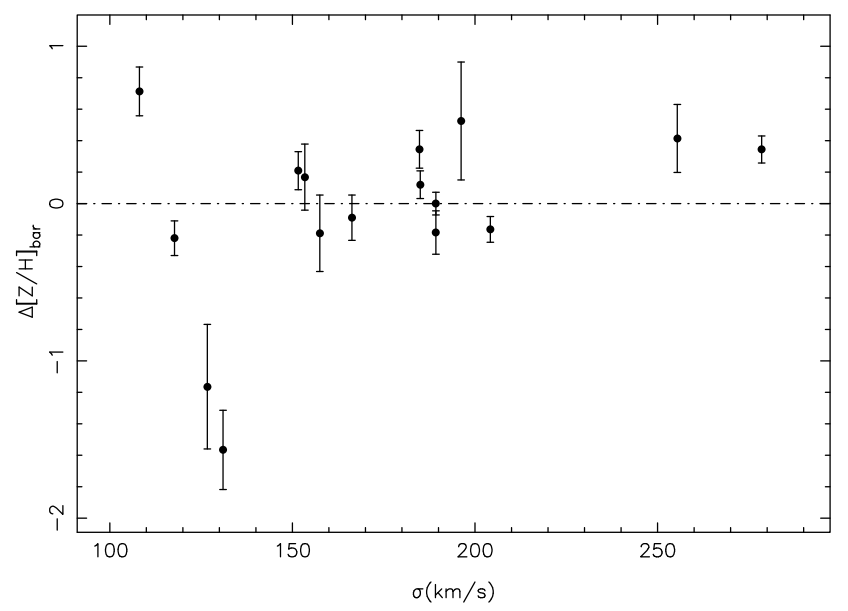

Fig. 11. Metallicity difference between the inner and the outer bar region as a function of maximum central velocity dispersion. Notice that galaxies with $\sigma<170 \mathrm{~km} \mathrm{~s}^{-1}$ tend to have negative gradient values.

NGC 2681, although belonging to the same case as NGC 2665, do not present any HII regions along their bars.

The lowest average bar value of the $\alpha / \mathrm{Fe}$, here expressed as $[\mathrm{E} / \mathrm{Fe}]$ (Trager et al. 2000), tends to have lower central velocity dispersion values (Fig. 9), these galaxies also in general have a lower average age (Fig. 9). The galaxies with higher central velocity dispersion stopped forming star earlier than galaxies with lower velocity dispersion. However, this is not an indication that the galaxies with lower central velocity dispersion host a younger (i.e. a more recently formed) bar.

\section{Conclusions}

We have measured, for the first time, line-strength indices along the bar of a sample of 20 early-type spiral galaxies and S0. We find that the mean values of age, metallicity and $[\mathrm{E} / \mathrm{Fe}]$ correlate with central velocity dispersion in a similar way to that of bulges, pointing to a intimate evolution of both components. Galaxies with high stellar velocity dispersions $\left(>170 \mathrm{~km} \mathrm{~s}^{-1}\right)$ host bars with old stars while galaxies with lower central velocity dispersion show stars with a large dispersion in their ages.

We find, for the first time, gradients in age and metallicity along the bars of all the galaxies. We have found three types of bars according to their metallicity and age distribution along the radius. Most galaxies could be compatible with having formed more than 3 Gyr ago, although a few of them show characteristics compatible with having been formed less than $<2$ Gyr ago. These derived gradients place strong constrains on models of bar evolution.

We have explored the possible correlation of the bar age with other bar/galaxy parameters, such as bar strength, bar size, morphological features, and AGN presence without finding any significant trend. All the galaxies show disk-like central components, implying a strong role played by bars in the bulge secular evolution. Among these disk-like central components we find some galaxies hosting an old, luminosity weighted, stellar component (>3 Gyr) and others with a recent $(<1$ Gyr) burst. Therefore, it would interesting to separate the bulge and inner disk populations to analyse their stellar population parameters in order to investigate the origin of the $\sigma$-drops. This will be presented in a forthcoming paper.
Acknowledgements. We are indebted to Dr. Marc Sarzi and Dr. Jesus Falcón-Barroso for their help and advices in the use of GANDALF. This work would not have been possible without it. We are also very gratefull to Dr. Jesús González for sharing his wisdom with us and to Dr. H. Wozniak for his useful comments which have greatly improved the manuscript. This research has made use of the NASA/IPAC Extragalactic Database (NED) which is operated by the Jet Propulsion Laboratory, California Institute of Technology, under contract with the National Aeronautics and Space Administration. We have also made use of the Digital Sky Survey. The Digitized Sky Surveys were produced at the Space Telescope Science Institute under U.S. Government grant NAG W-2166. The images of these surveys are based on photographic data obtained using the Oschin Schmidt Telescope on Palomar Mountain and the UK Schmidt Telescope. I. Pérez is supported by a postdoctoral fellowship from the Netherlands Organisation for Scientific Research (NWO, VeniGrant 639.041.511) and the Spanish Plan Nacional del Espacio del Ministerio de Educación y Ciencia. P.S.B is supported by a Marie Curie Intra-European Fellowship within the 6th European Community Framework Programme. I.P. thanks support for a visit to the University of Central Lancashire from a Livesey Award. A.Z. acknowledges support from the Consejería de Eduación y Ciencia de la Junta de Andalucía

\section{References}

Abraham, R. G., Merrifield, M. R., Ellis, R. S., Tanvir, N. R., \& Brinchmann, J. 1999, MNRAS, 308, 569

Athanassoula, E. 2005, Celestial Mechanics and Dynamical Astronomy, 91, 9

Berentzen, I., Heller, C. H., Shlosman, I., \& Fricke, K. J. 1998, MNRAS, 300, 49

Berentzen, I., Shlosman, I., Martinez-Valpuesta, I., \& Clayton, H. 2007, ApJ, 666, 189

Block, D., Puerari, I., Knapen, J., et al. 2001, A\&A, 375, 761

Block, D., Buta, R., Knapen, J., Elmegreen, D., \& Puerari, I. 2004, AJ, 128, 183

Bono, G., Caputo, F., Cassisi, S., Castellani, V., \& Marconi, M. 1997, ApJ, 489, 822

Bournaud, F., \& Combes, F. 2002, A\&A, 392, 83

Buta, R. 1986, ApJS, 61, 609

Buta, R., \& Block, D. L. 2001, ApJ, 550, 243

Buta, R., Laurikainen, E., Salo, H., Block, D., \& Knapen, J. 2006, AJ, 132, 1859

Cardelli, J. A., Clayton, G. C., \& Mathis, J. S. 1989, in Interstellar Dust, ed. L. J. Allamandola, \& A. G. G. M. Tielens, IAU Symp., 135, 5P

Cardiel, N. 1999, Ph.D. Thesis

Cardiel, N., Gorgas, J., Cenarro, J., \& Gonzalez, J. J. 1998, A\&AS, 127, 597

Cassisi, S., Castellani, M., \& Castellani, V. 1997, A\&A, 317, 108

Cassisi, S., Castellani, V., degl'Innocenti, S., Salaris, M., \& Weiss, A. 1999, A\&AS, 134, 103

Chilingarian, I. V., Cayatte, V., Durret, F., et al. 2008, A\&A, 486, 85

Chung, A., \& Bureau, M. 2004, AJ, 127, 3192

Combes, F., \& Sanders, R. 1981, A\&A, 96, 164

de Vaucouleurs, G. 1948, Ann. Astrophys., 11, 24

Elmegreen, B. G., Elmegreen, D. M., \& Hirst, A. C. 2004, ApJ, 612, 191

Erwin, P. 2004, A\&A, 415, 941

Erwin, P. 2005, MNRAS, 364, 283

Eskridge, P. B., Frogel, J. A., Pogge, R. W., et al. 2000, AJ, 119, 536

Faber, S. M., Worthey, G., \& Gonzales, J. J. 1992, in The Stellar Populations of Galaxies, ed. B. Barbuy, \& A. Renzini, IAU Symp., 149, 255

Ferrarese, L., \& Ford, H. 2005, Space Sci. Rev., 116, 523

Friedli, D., \& Benz, W. 1993, A\&A, 268, 65

Friedli, D., \& Benz, W. 1995, A\&A, 301, 649

Friedli, D., \& Pfenniger, D. 1991, in Dynamics of Galaxies and Their Molecular Cloud Distributions, ed. F. Combes, \& F. Casoli, IAU Symp., 146, 362

Friedli, D., Benz, W., \& Kennicutt, R. 1994, ApJ, 430, L105

Friedli, D., Wozniak, H., Rieke, M., Martinet, L., \& Bratschi, P. 1996, A\&AS, 118,461

Gadotti, D., \& de Souza, R. 2006, ApJS, 163, 270

Gadotti, D. A., \& de Souza, R. E. 2006, ApJS, 163, 270

Girardi, L., Bressan, A., Bertelli, G., \& Chiosi, C. 2000, A\&AS, 141, 371

Gorgas, J., Faber, S., Burstein, D., et al. 1993, ApJS, 86, 153

Hameed, S., \& Devereux, N. 1999, AJ, 118, 730

Jogee, S., Barazza, F. D., Rix, H.-W., et al. 2004, ApJ, 615, L105

Jungwiert, B., Combes, F., \& Axon, D. J. 1997, A\&AS, 125, 479

King, D. L. 1985, RGO/La Palma technical note, 31

Knapen, J., Stedman, S., Bramich, D., Folke, S., \& Bradley, T. 2004, A\&A, 423, 1135

Knapen, J. H., Shlosman, I., \& Peletier, R. F. 2000, ApJ, 529, 93

Korn, A. J. 2004, in Origin and Evolution of the Elements, ed. A. McWilliam, \& M. Rauch 
Kuntschner, H. 2000, MNRAS, 315, 184

Laurikainen, E., Salo, H., Buta, R., \& Vasylyev, S. 2004, MNRAS

MacArthur, L. A. 2005, ApJ, 623, 795

Marinova, I., \& Jogee, S. 2007, ApJ, 659, 1176

Márquez, I., Masegosa, J., Durret, F., et al. 2003, A\&A, 409, 459

Martin, P., \& Friedli, D. 1997, A\&A, 326, 449

Martin, P., \& Friedli, D. 1999, A\&A, 346, 769

Martin, P., \& Roy, J.-R. 1995, ApJ, 445, 161

Menéndez-Delmestre, K., Sheth, K., Schinnerer, E., Jarrett, T. H., \& Scoville, N. Z. 2007, ApJ, 657, 790

Michel-Dansac, L., \& Wozniak, H. 2006, A\&A, 452, 97

Mollá, M., Hardy, E., \& Beauchamp, D. 1999, AJ, 513, 695

Moorthy, B., \& Holtzman, J. 2006, MNRAS, 371, 583

Mulchaey, J. S., Regan, M. W., \& Kundu, A. 1997, ApJS, 110, 299

Norman, C. A., Sellwood, J. A., \& Hasan, H. 1996, ApJ, 462, 114

Oconnell, R. W. 1976, ApJ, 206, 370

Peletier, R., Falcón-Barroso, J., Bacon, R., et al. 2007, MNRAS, 379, 445

Pérez, I., Márquez, I., Freeman, K., \& Fux, R. 2005, A\&A, 438, 127

Pérez, I., Sánchez-Blázquez, P., \& Zurita, A. 2007, A\&A, 465, 9

Phillips, A. C. 1996, in Barred Galaxies, ed. R. Buta, D. A. Crocker, \& B. G. Elmegreen, ASP Conf. Ser., 91, IAU Coll., 157, 44

Proctor, R. N., \& Sansom, A. E. 2002, MNRAS, 333, 517

Regan, M. W., Teuben, P. J., Vogel, S. N., \& van der Hulst, T. 1996, AJ, 112, 2549

Renzini, A., \& Buzzoni, A. 1986, in Spectral Evolution of Galaxies, ed. C. Chiosi, \& A. Renzini, Ap\&SS Library, 122, 195

Rodgers, A. W., Conroy, P., \& Bloxham, G. 1988, PASP, 100, 626
Ryder, S., Fenner, Y., \& Gibson, B. 2005, MNRAS, 358, 1337

Salasnich, B., Girardi, L., Weiss, A., \& Chiosi, C. 2000, A\&A, 361, 1023

Sánchez-Blázquez, P., Peletier, R., Jiménez-Vicente, J., et al. 2006, MNRAS, 371,703

Scoville, N., Aussel, H., Brusa, M., et al. 2007, ApJS, 172, 1

Serra, P., \& Trager, S. 2007, MNRAS, 374, 769

Shen, J., \& Sellwood, J. A. 2004, ApJ, 604, 614

Sheth, K., Elmegreen, D. M., Elmegreen, B. G., et al. 2008, ApJ, 675, 1141

Sholsman, I., Juhan, F. \& Begelman, M. C. 1989, Nature, 338, 45

Tantalo, R., Chiosi, C., \& Bressan, A. 1998, A\&A, 333, 419

Thomas, D., Maraston, C., \& Bender, R. 2003, MNRAS, 339, 897

Trager, S. C., Worthey, G., Faber, S. M., Burstein, D., \& Gonzalez, J. J. 1998, ApJS, 116, 1

Trager, S. C., Faber, S. M., Worthey, G., \& González, J. J. 2000, AJ, 119, 1645

van den Bergh, S., Abraham, R. G., Whyte, L. F., et al. 2002, AJ, 123, 2913

Verley, S., Leon, S., Verdes-Montenegro, L., et al. 2007, A\&A, 472, 121

Verón-Cetty, M.-P., \& Véron, P. 2006, A\&A, 455, 773

Whyte, L. F., Abraham, R. G., Merrifield, M. R., et al. 2002, MNRAS, 336, 1281

Worthey, G. 1994, ApJS, 95, 107

Worthey, G., \& Ottaviani, D. L. 1997, ApJS, 111, 377

Wozniak, H. 2007, A\&A, 465, 1

Wozniak, H., \& Champavert, N. 2006, MNRAS, 369, 853

Wozniak, H., Friedli, D., Martinet, L., Martin, P., \& Bratschi, P. 1995, A\&AS, 111,115

Yoachim, P., \& Dalcanton, J. J. 2008, ApJ, 683, 707

Zurita, A., \& Pérez, I. 2008, A\&A, 485, 5 
I. Pérez et al.: Study of the stellar line-strength indices and kinematics along bars. I., Online Material $p 1$

\section{Appendix A: Line-strength distribution}

Figure A.1 shows the line-strength gradients in the bar region for all the galaxies.
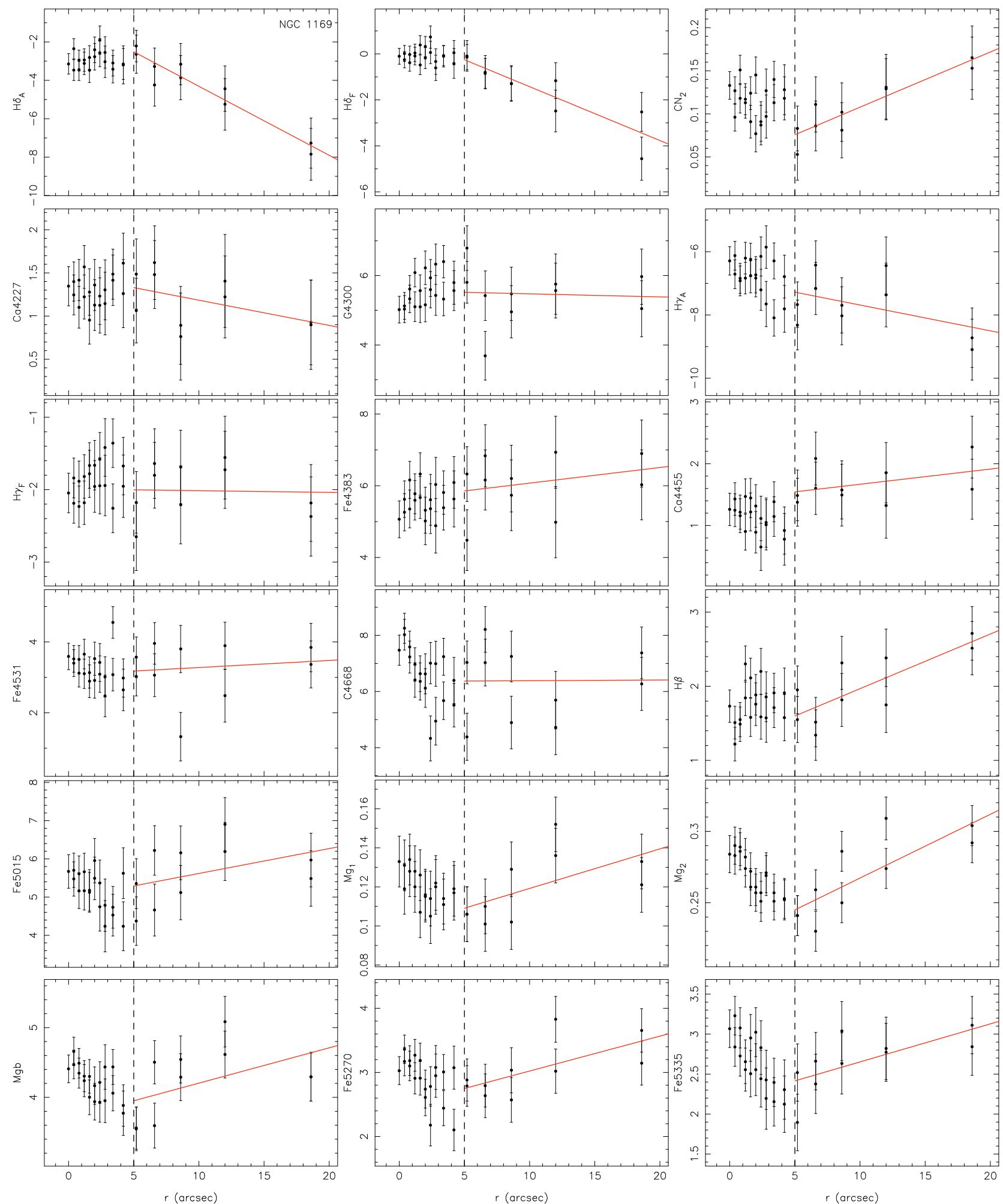

Fig. A.1. Line-strength distribution in the bar region for all the galaxies.

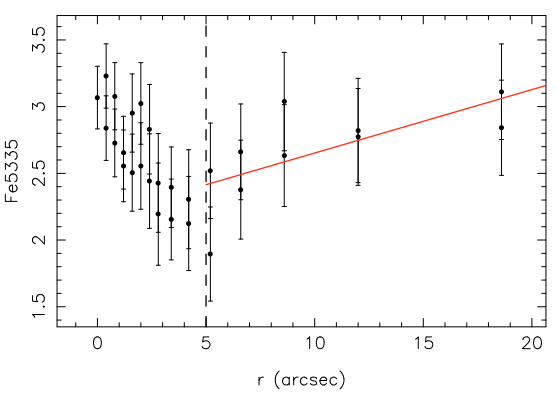

\footnotetext{
Fig. A.1. Line-strength distribution in the bar region for all the galaxies.
} 
I. Pérez et al.: Study of the stellar line-strength indices and kinematics along bars. I., Online Material p 2
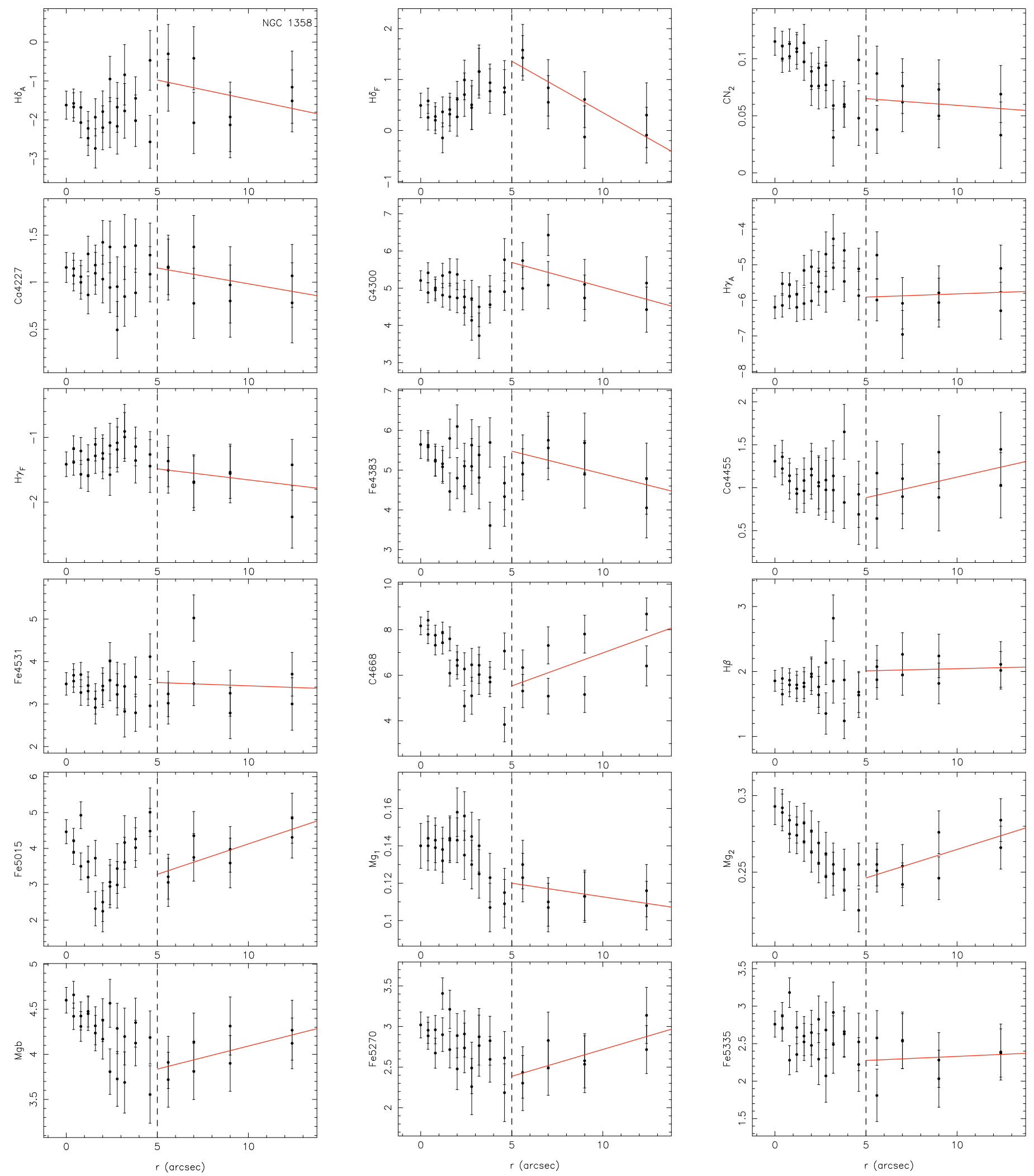

Fig. A.1. continued. 
I. Pérez et al.: Study of the stellar line-strength indices and kinematics along bars. I., Online Material p 3
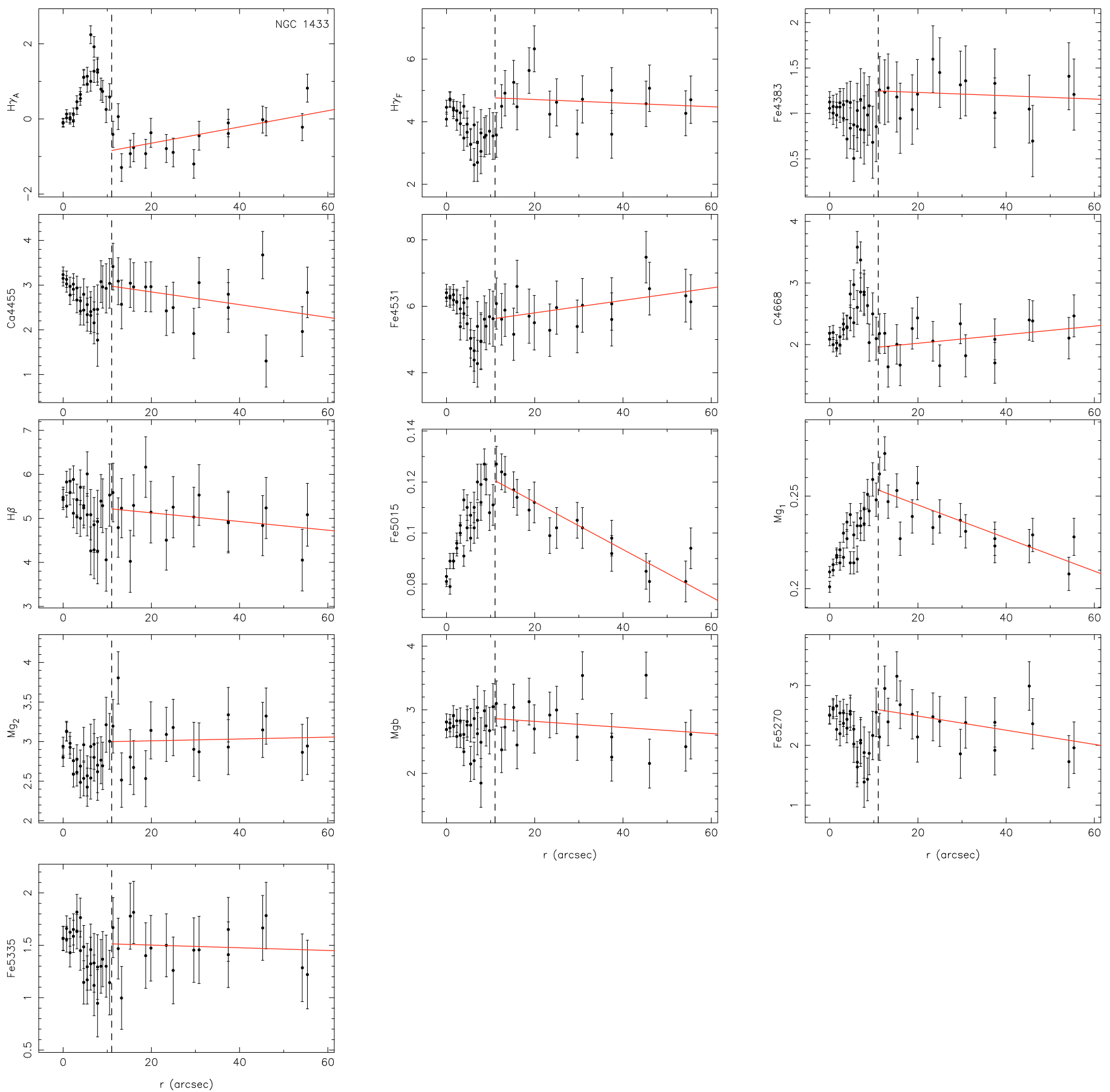

Fig. A.1. continued. 
I. Pérez et al.: Study of the stellar line-strength indices and kinematics along bars. I., Online Material p 4
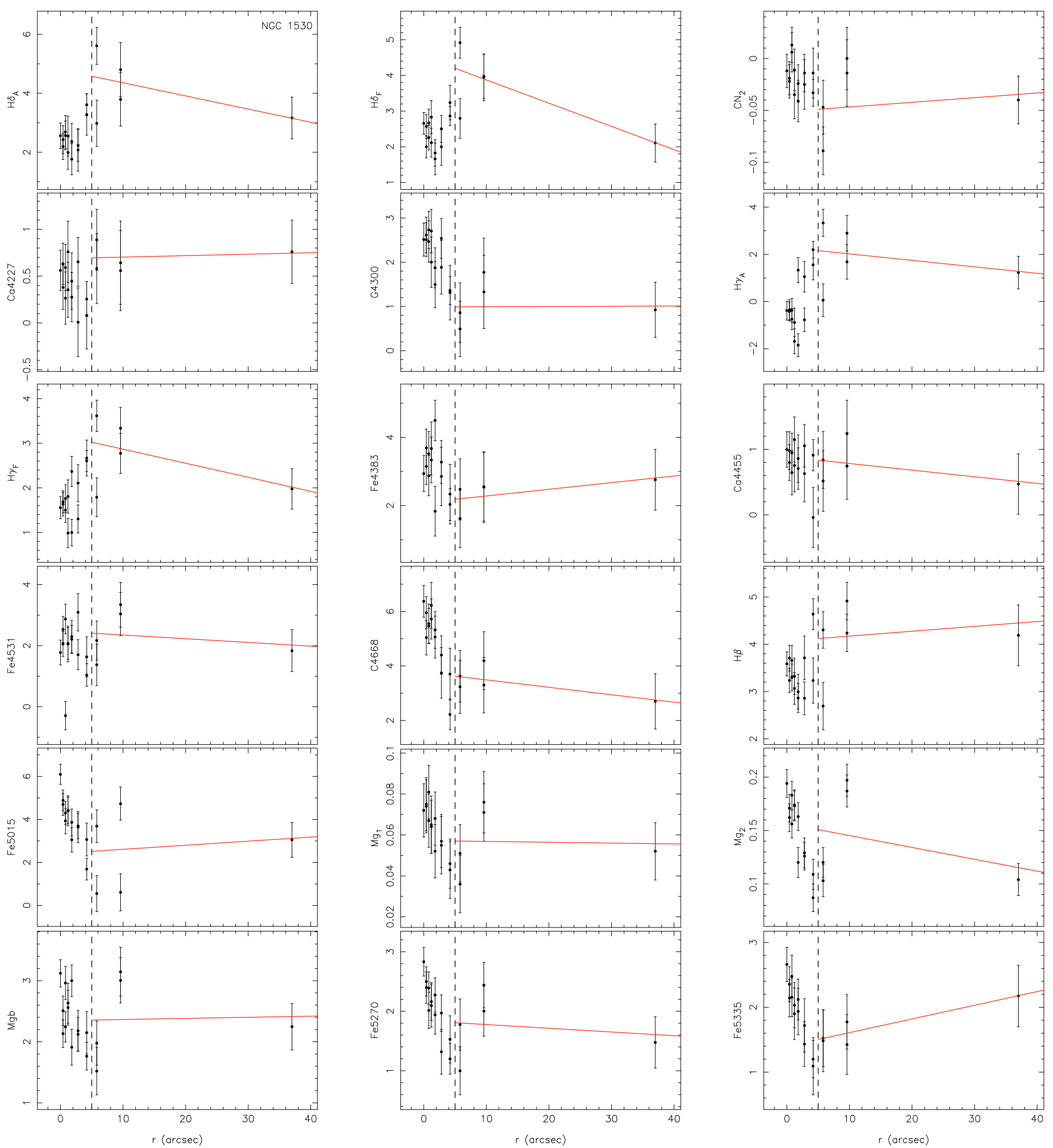

Fig. A.1. continued. 
I. Pérez et al.: Study of the stellar line-strength indices and kinematics along bars. I., Online Material p 5
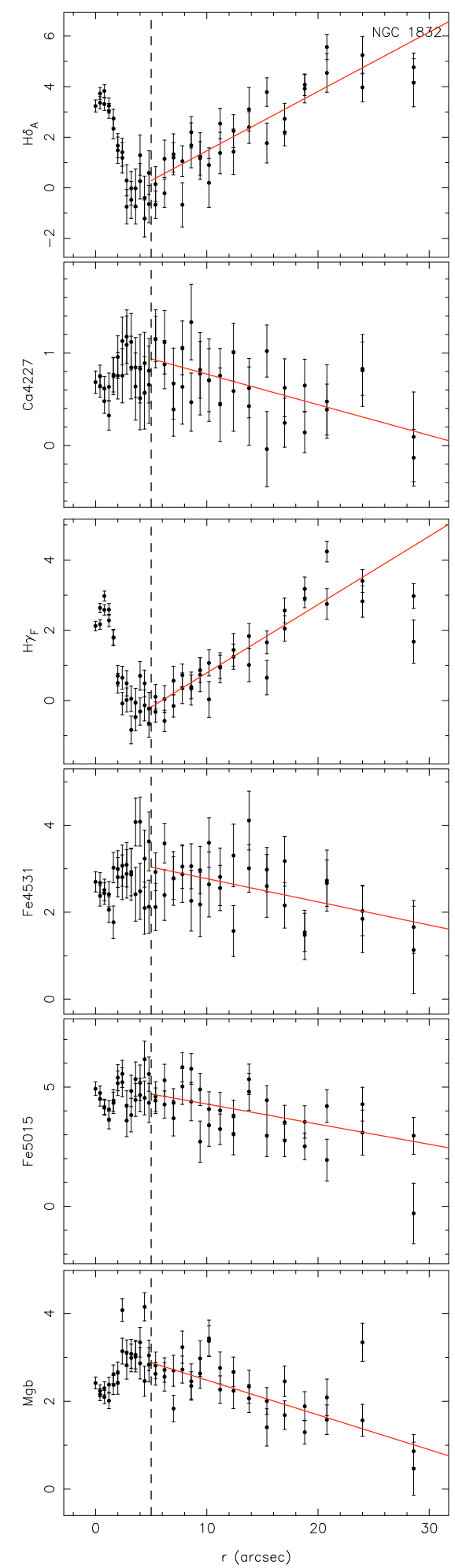

Fig. A.1. continued.
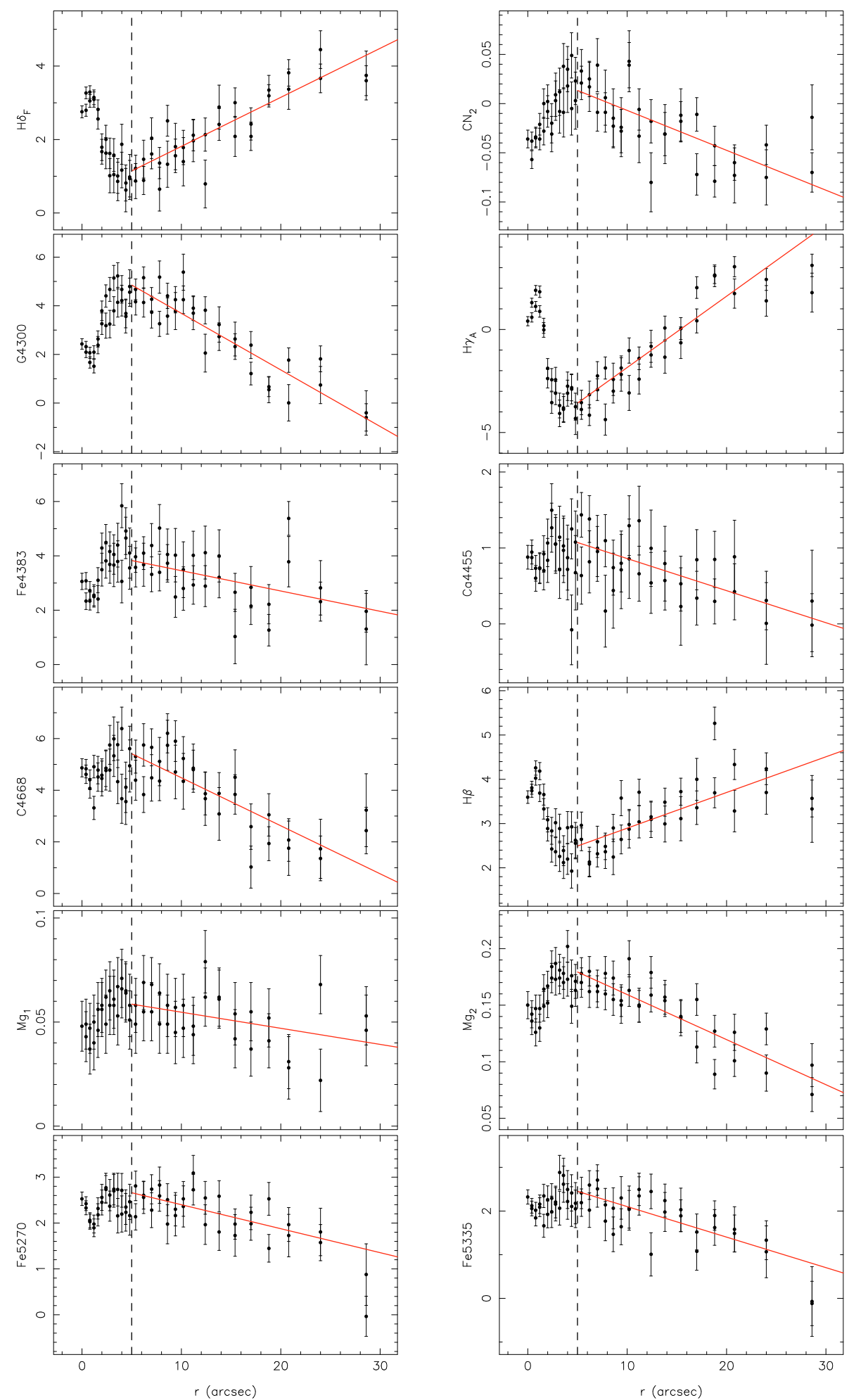
I. Pérez et al.: Study of the stellar line-strength indices and kinematics along bars. I., Online Material $p 6$
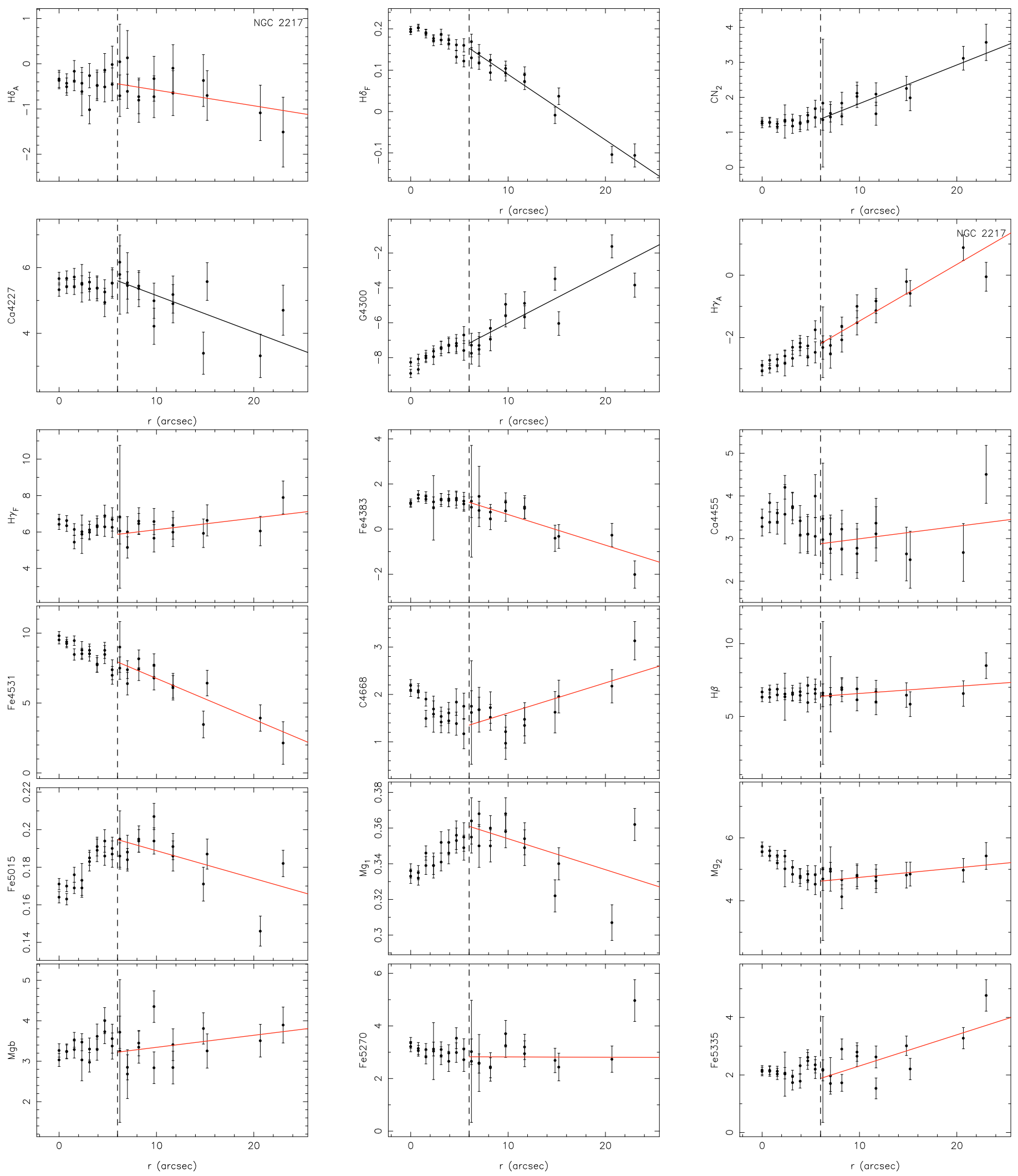

Fig. A.1. continued. 
I. Pérez et al.: Study of the stellar line-strength indices and kinematics along bars. I., Online Material $p 7$
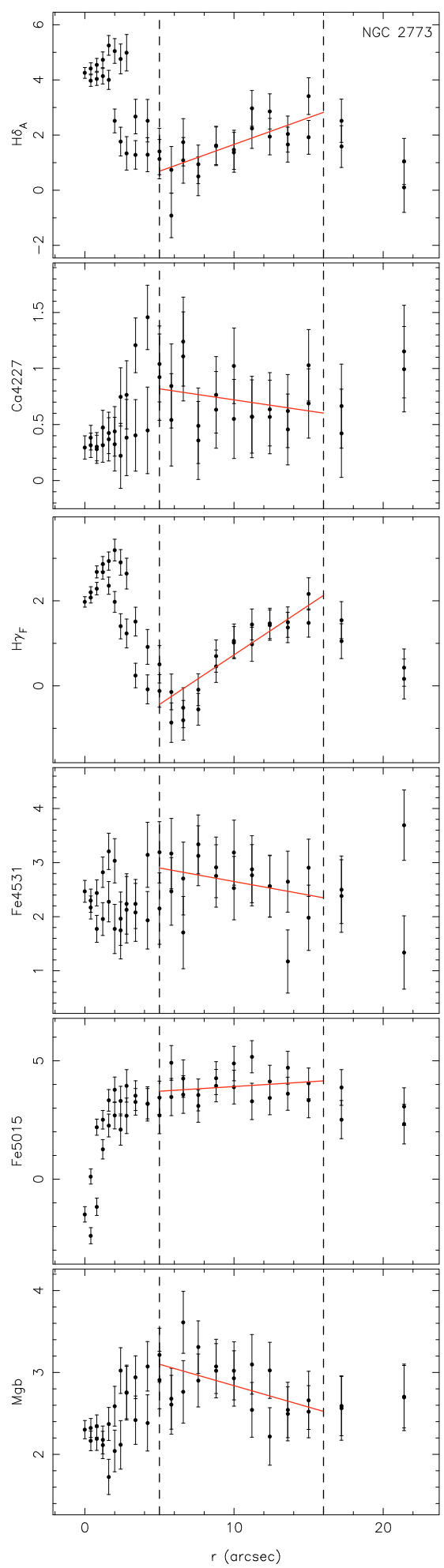

Fig. A.1. continued.
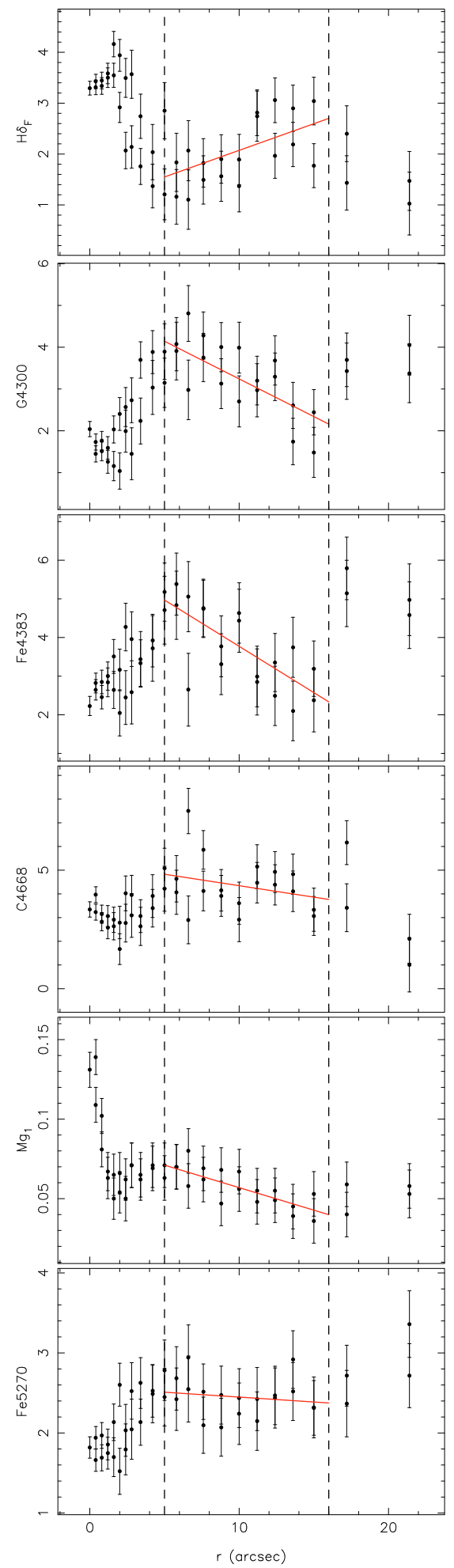
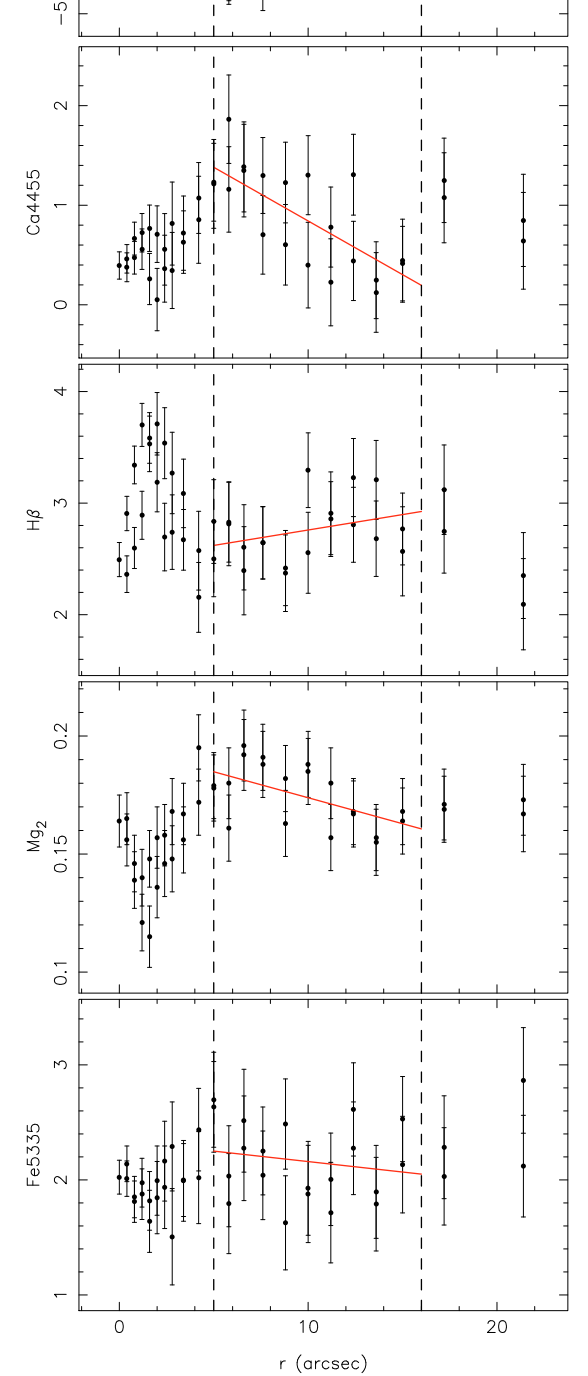
I. Pérez et al.: Study of the stellar line-strength indices and kinematics along bars. I., Online Material $p 8$
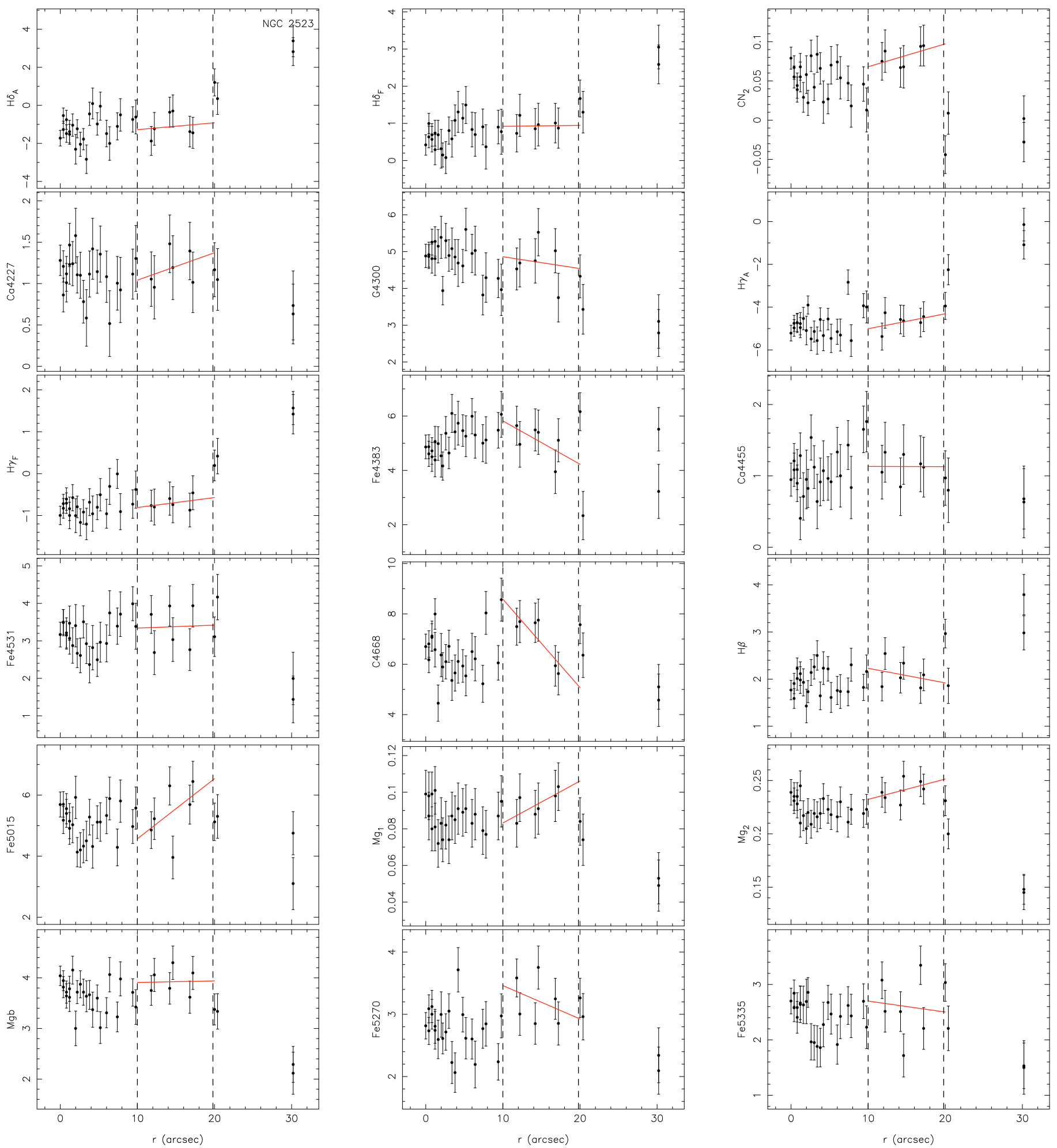

Fig. A.1. continued. 
I. Pérez et al.: Study of the stellar line-strength indices and kinematics along bars. I., Online Material $p 9$
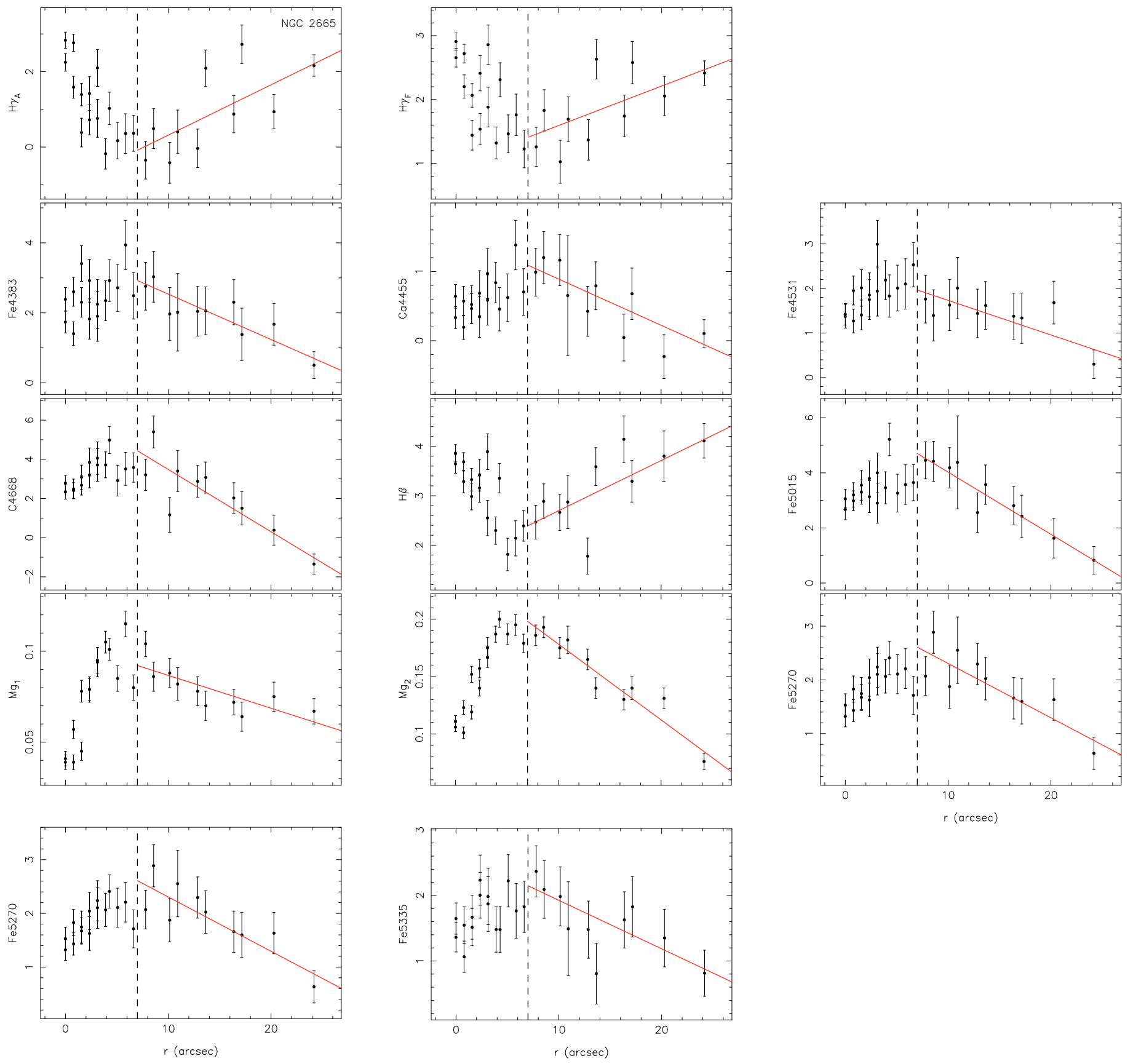

Fig. A.1. continued. 
I. Pérez et al.: Study of the stellar line-strength indices and kinematics along bars. I., Online Material p 10
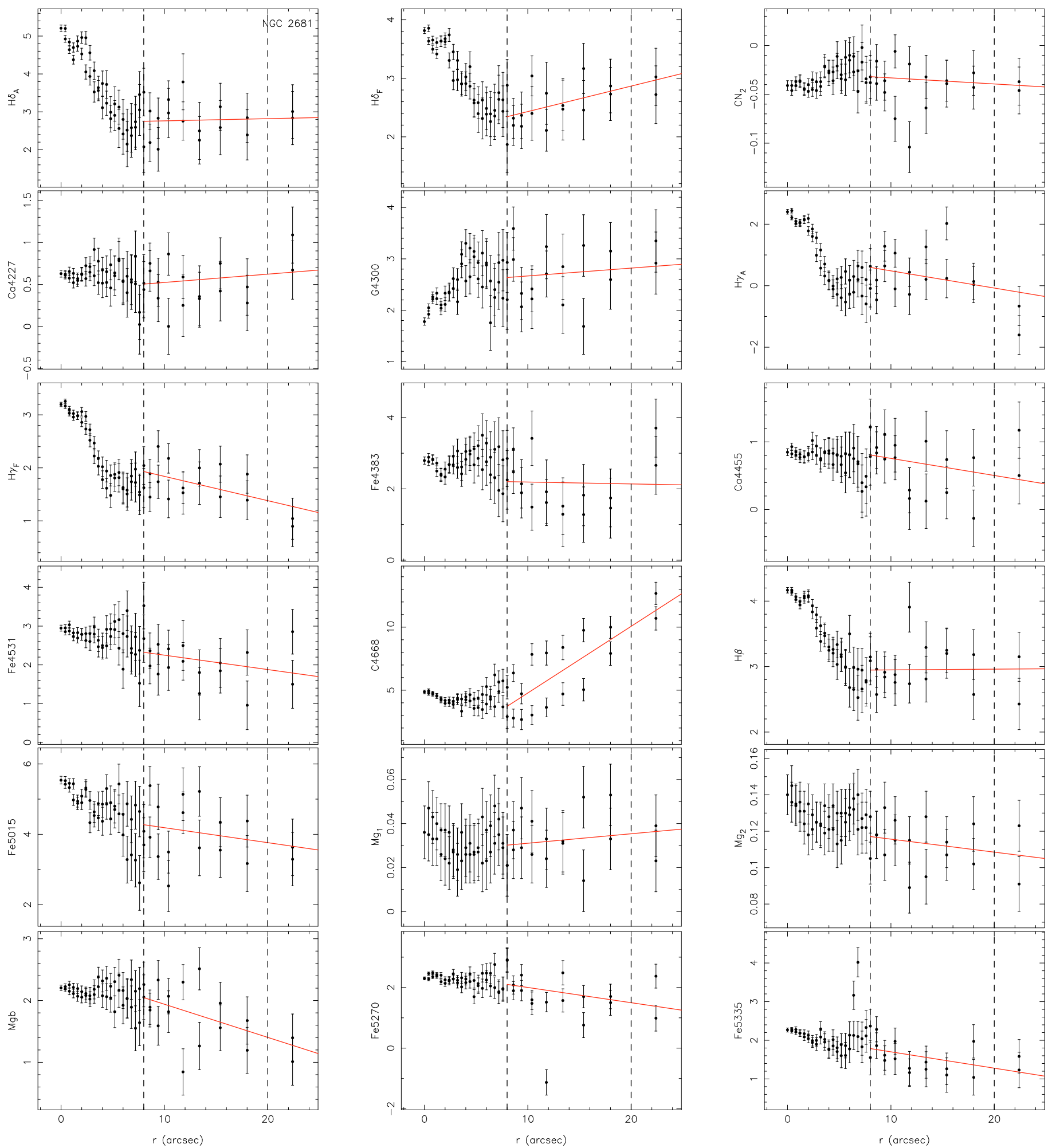

Fig. A.1. continued. 
I. Pérez et al.: Study of the stellar line-strength indices and kinematics along bars. I., Online Material $p 11$
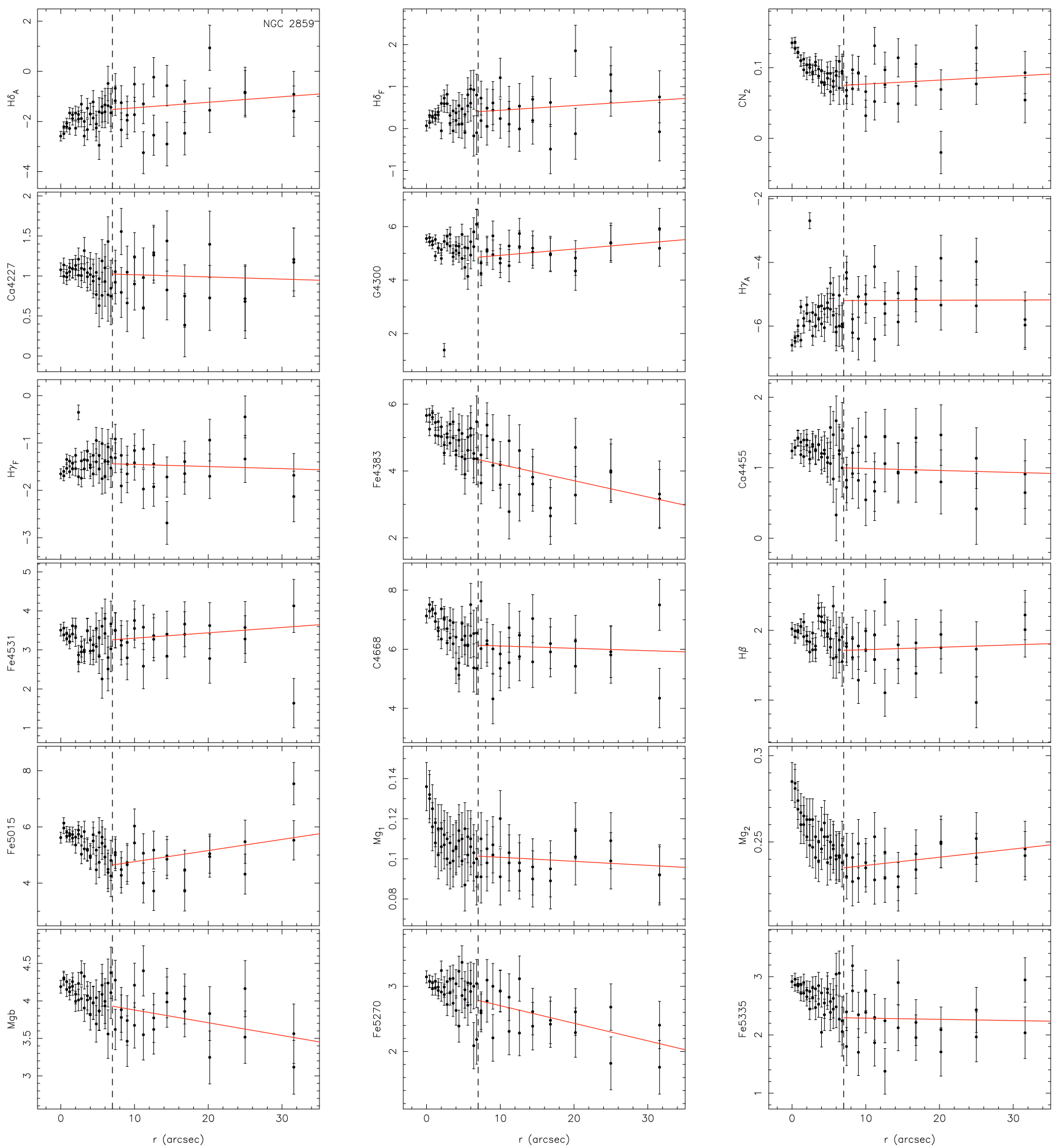

Fig. A.1. continued. 
I. Pérez et al.: Study of the stellar line-strength indices and kinematics along bars. I., Online Material p 12
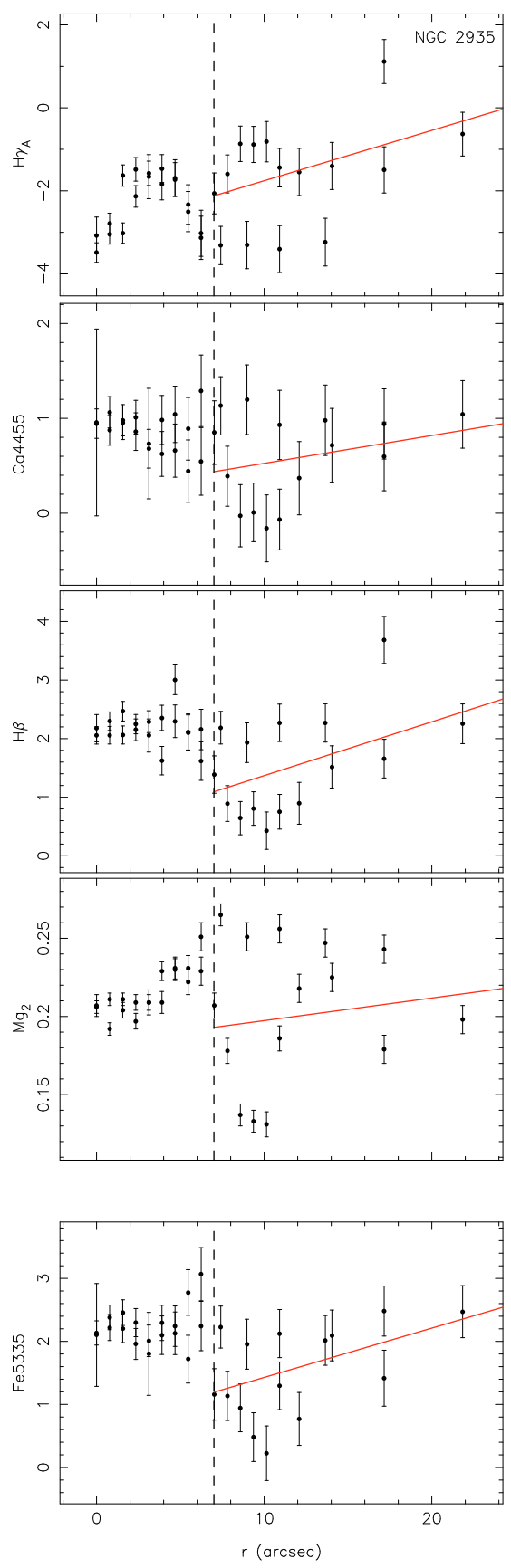

Fig. A.1. continued.
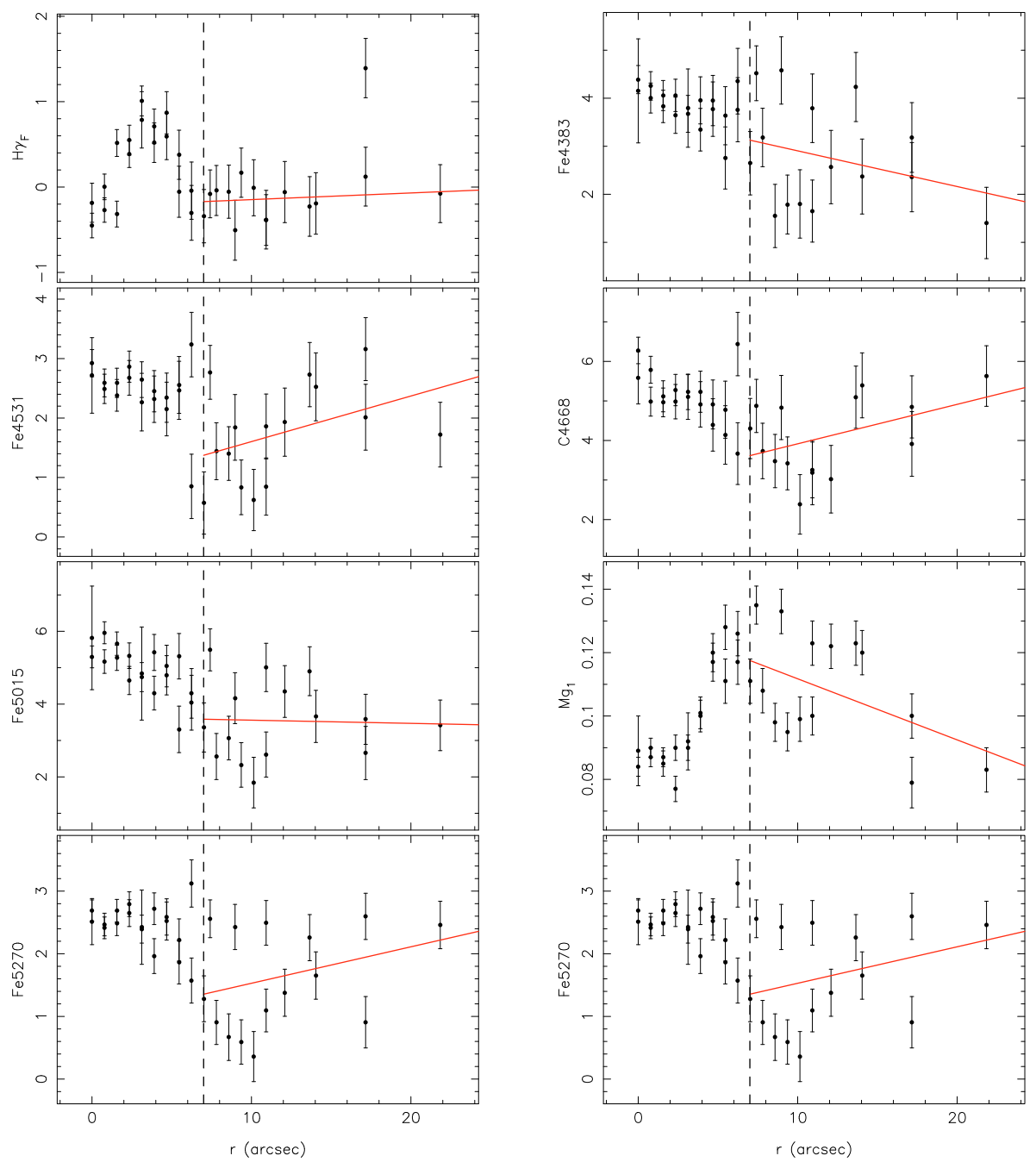
I. Pérez et al.: Study of the stellar line-strength indices and kinematics along bars. I., Online Material p 13
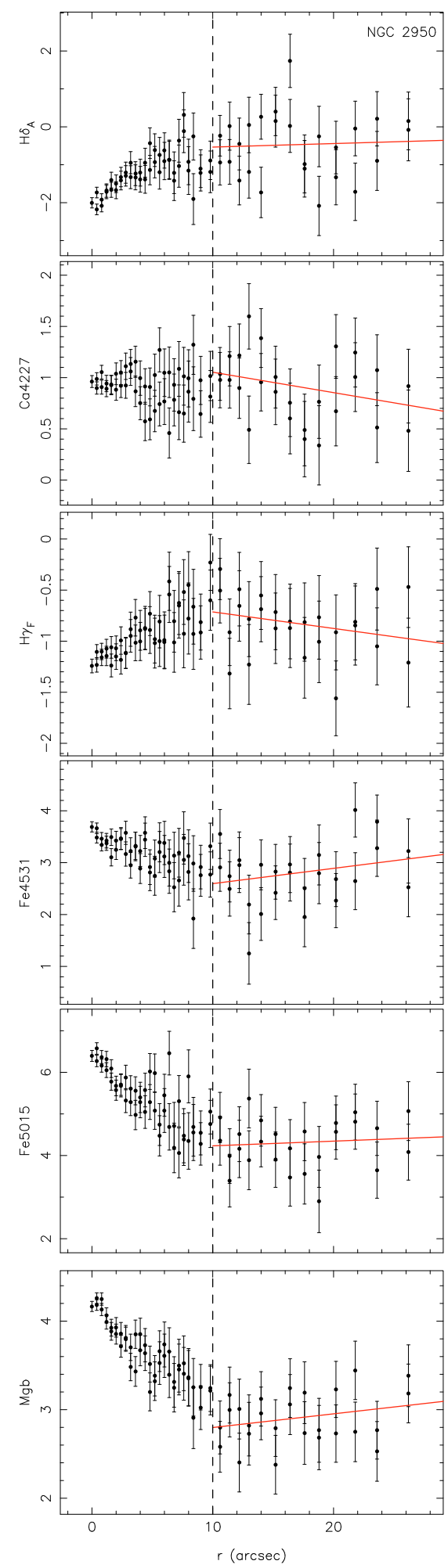

Fig. A.1. continued.
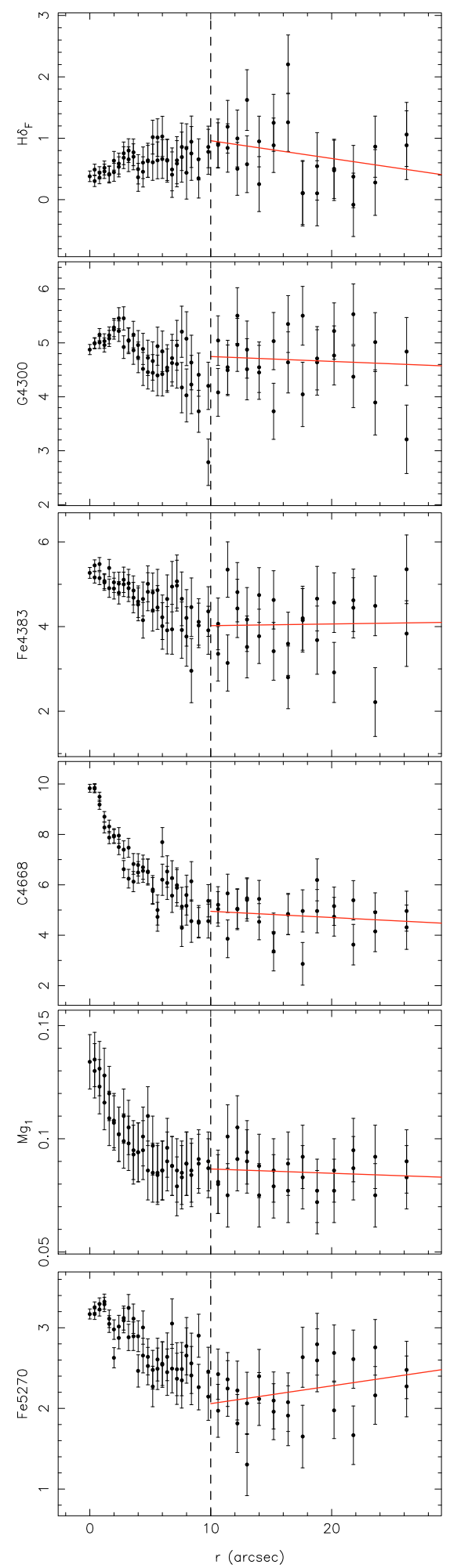
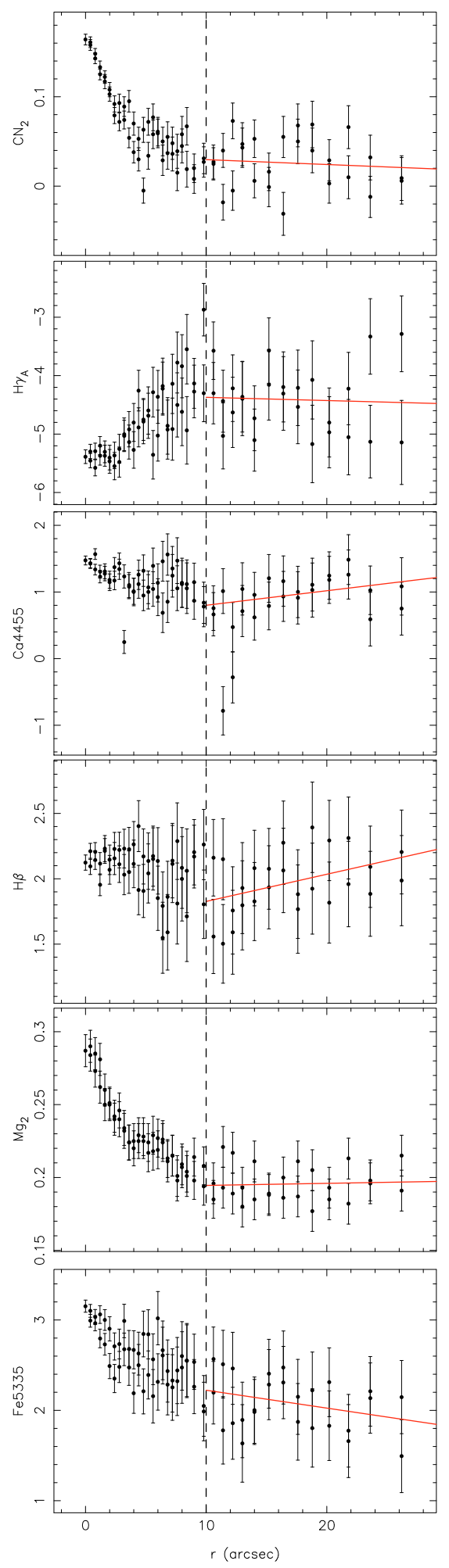
I. Pérez et al.: Study of the stellar line-strength indices and kinematics along bars. I., Online Material p 14
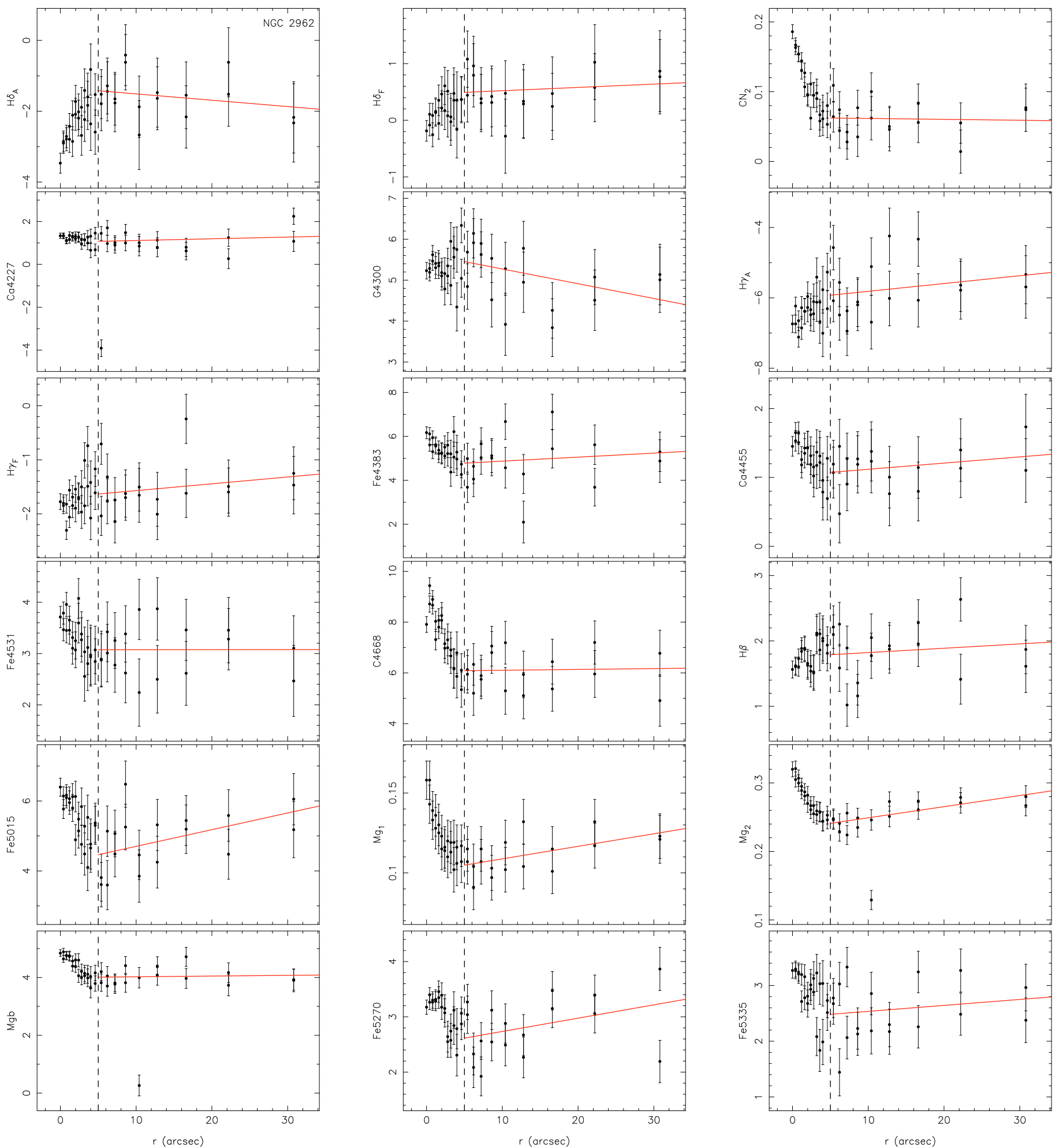

Fig. A.1. continued. 
I. Pérez et al.: Study of the stellar line-strength indices and kinematics along bars. I., Online Material $p 15$
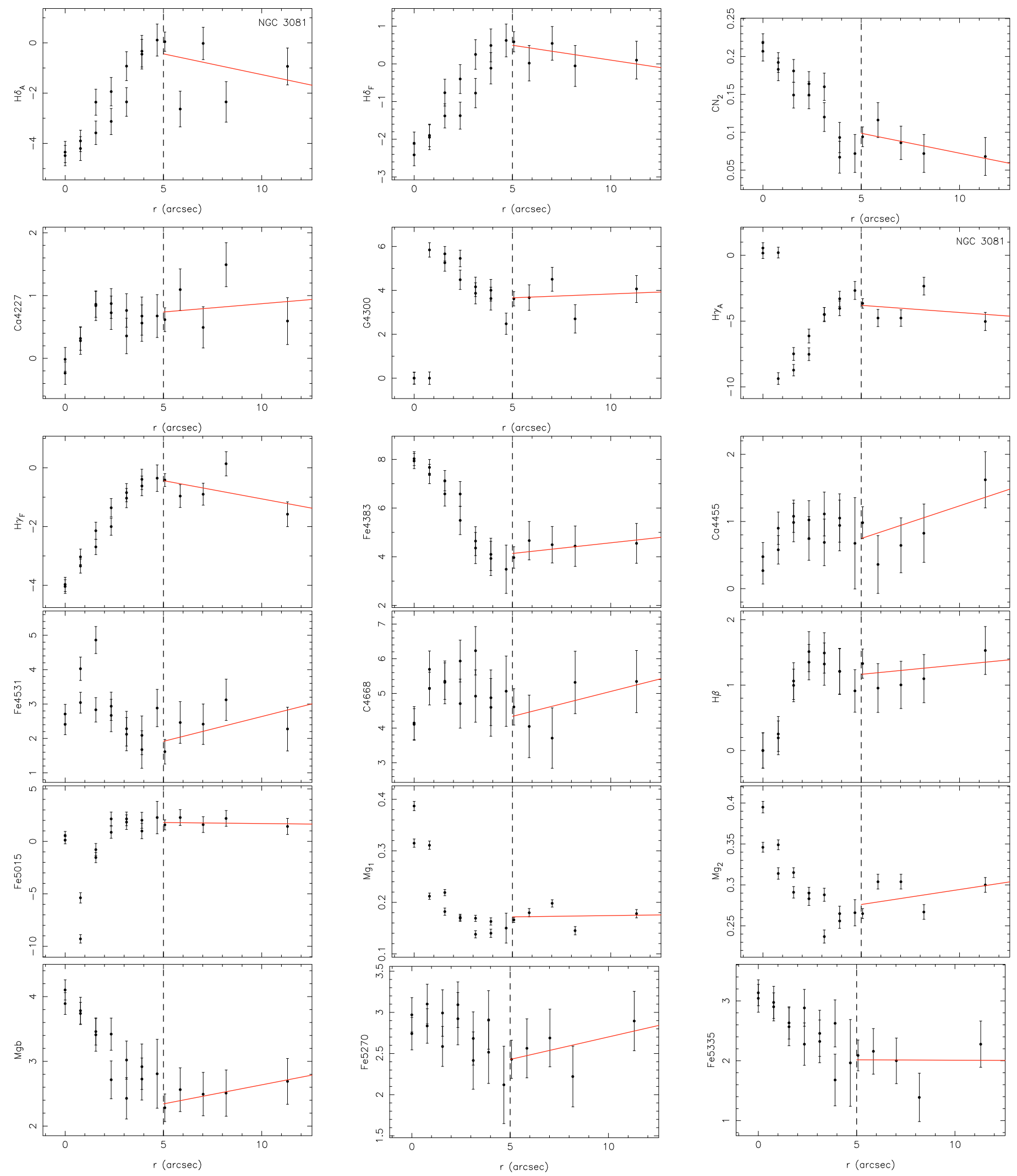

Fig. A.1. continued. 
I. Pérez et al.: Study of the stellar line-strength indices and kinematics along bars. I., Online Material p 16
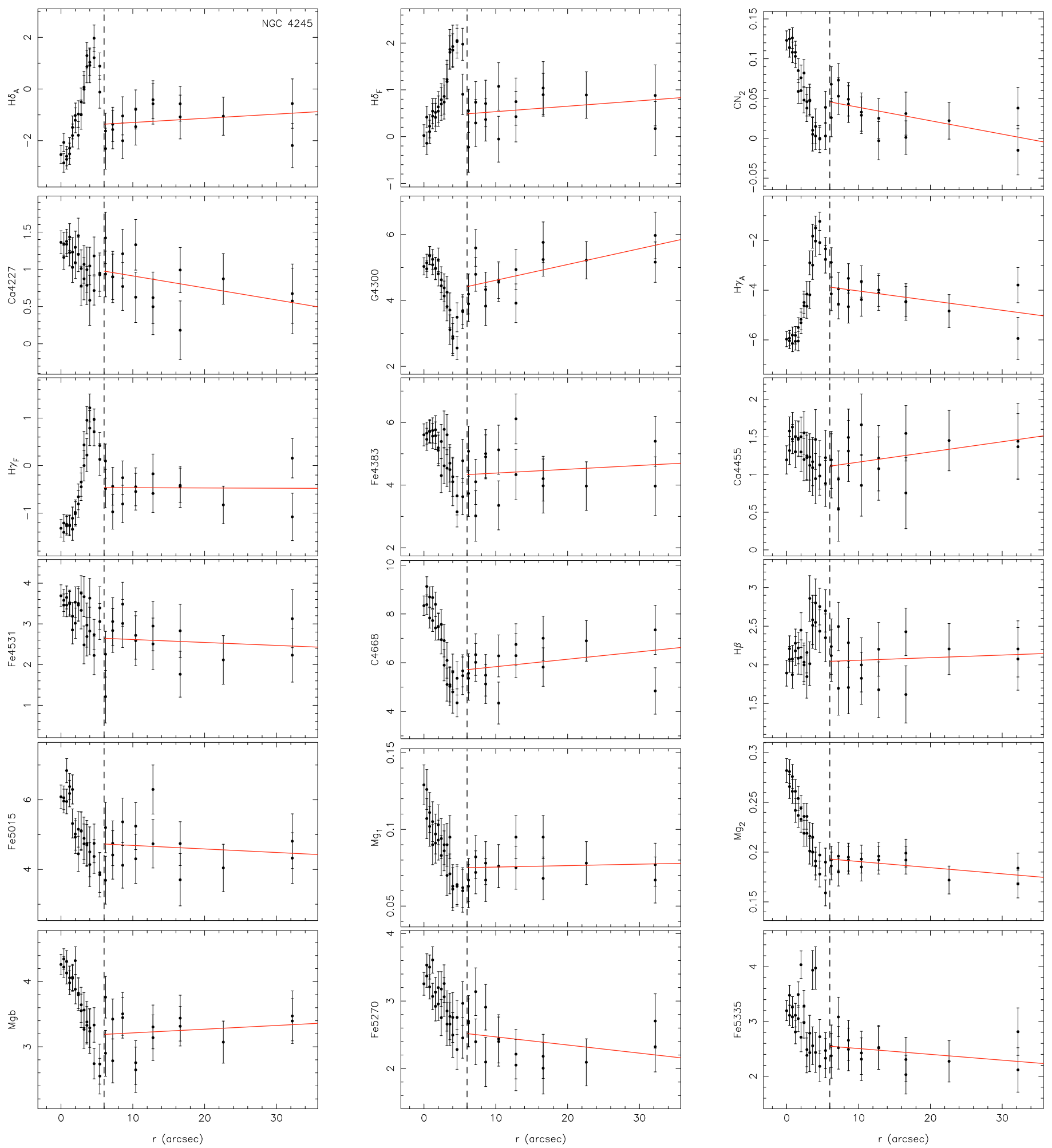

Fig. A.1. continued. 
I. Pérez et al.: Study of the stellar line-strength indices and kinematics along bars. I., Online Material $p 17$
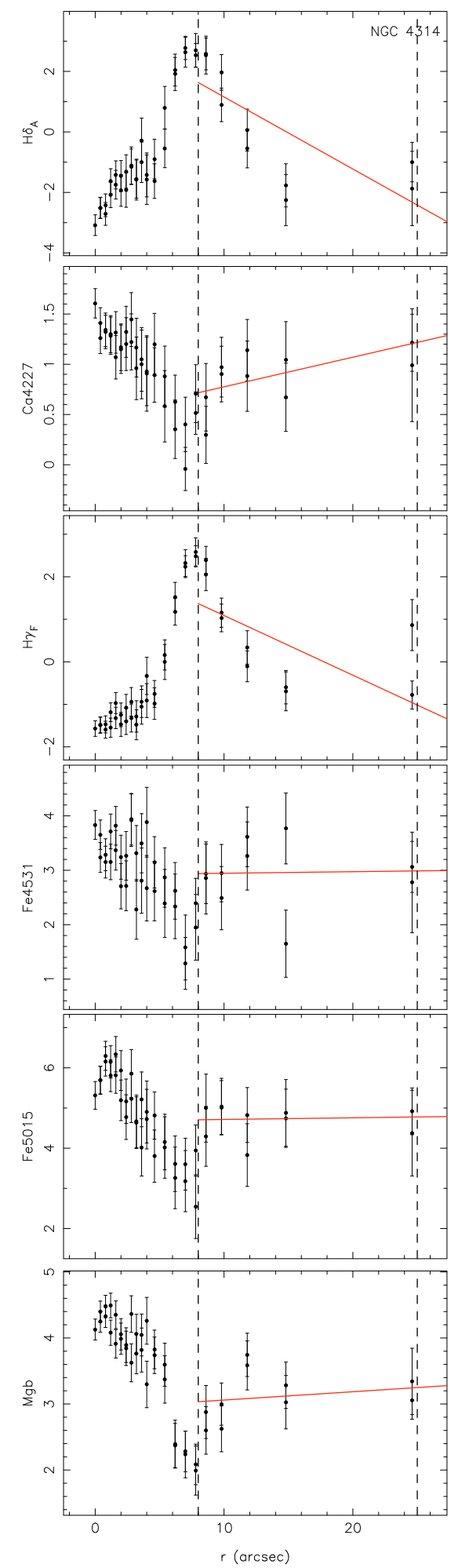

Fig. A.1. continued.
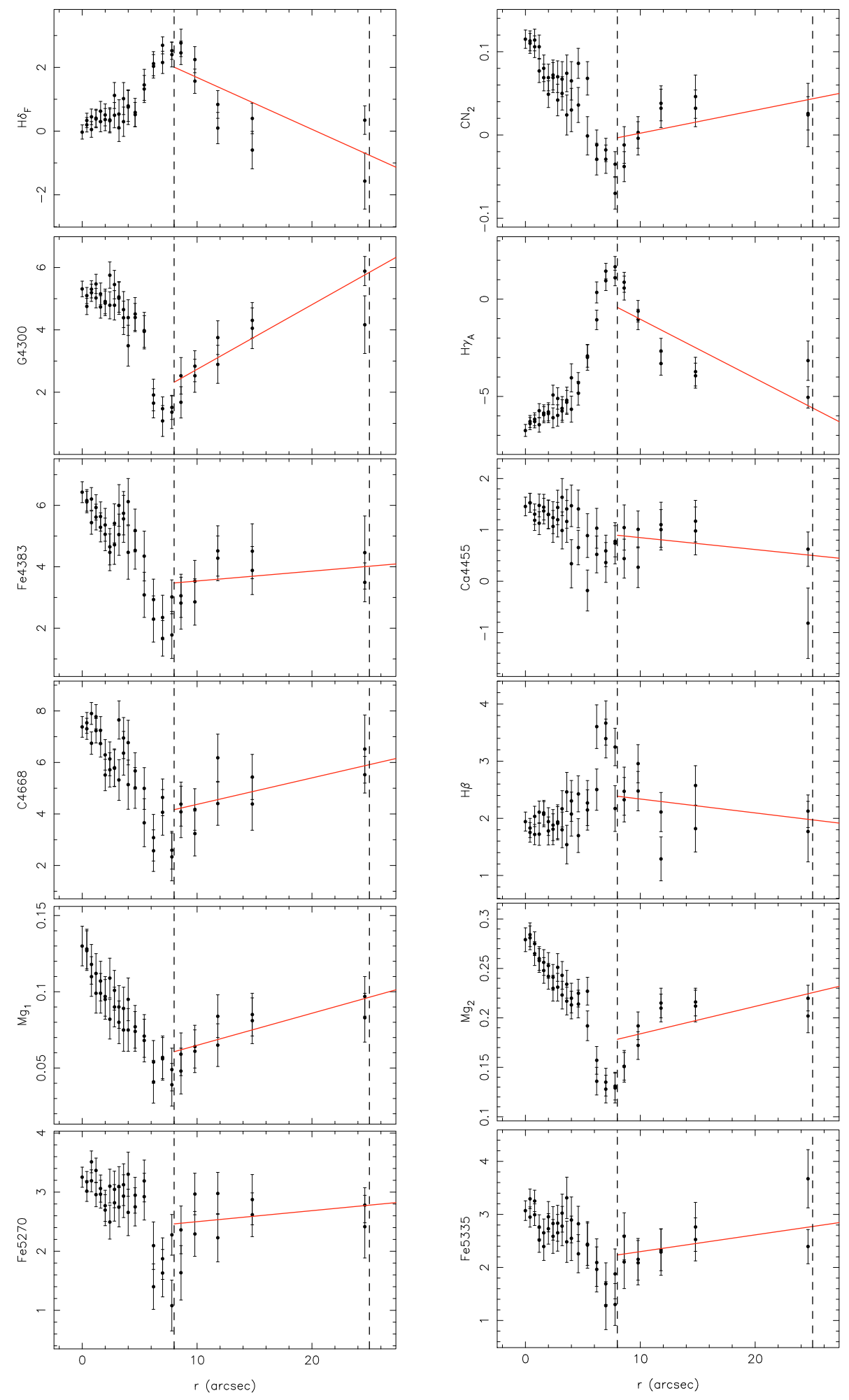
I. Pérez et al.: Study of the stellar line-strength indices and kinematics along bars. I., Online Material p 18
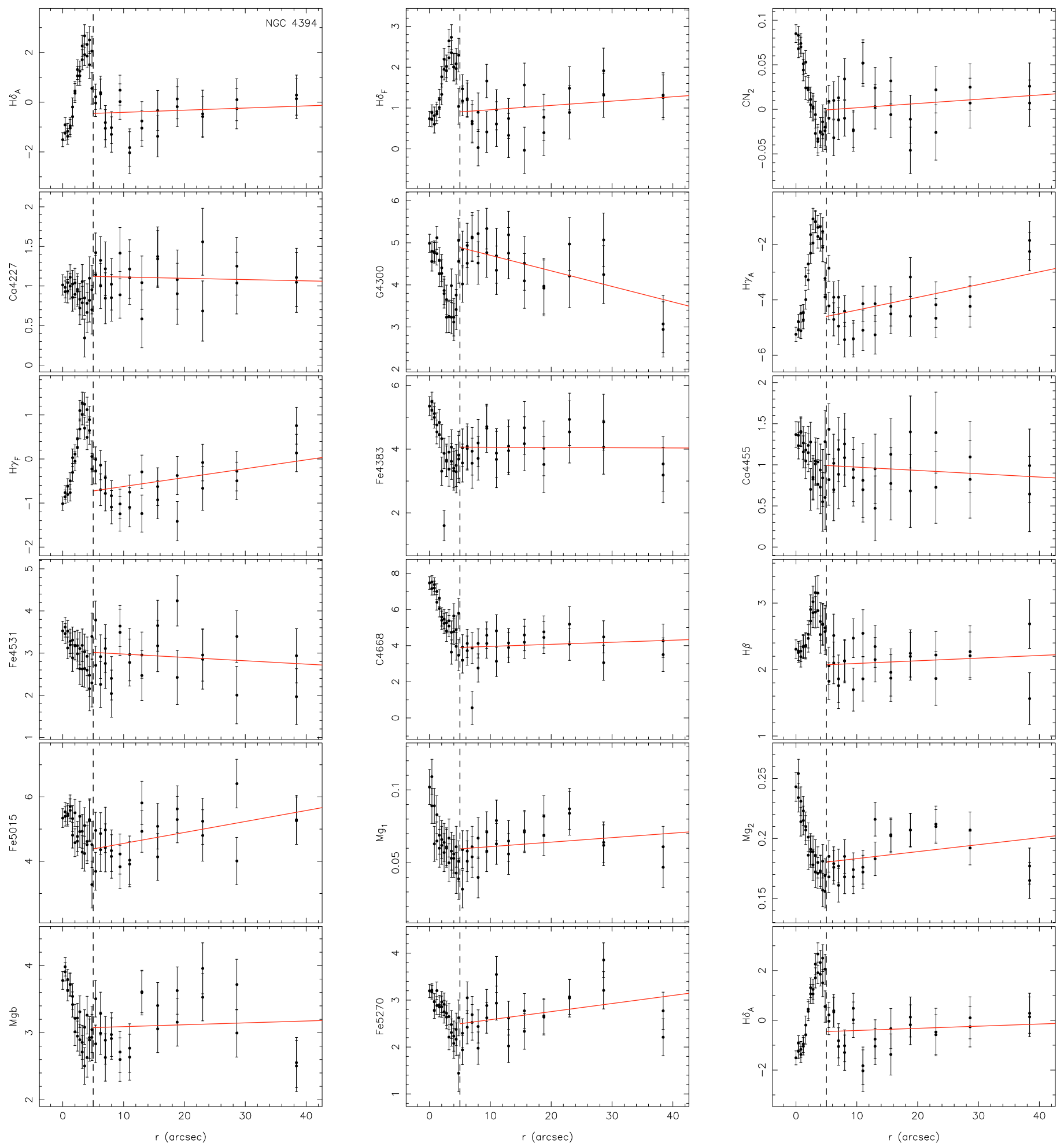

Fig. A.1. continued. 
I. Pérez et al.: Study of the stellar line-strength indices and kinematics along bars. I., Online Material $p 19$
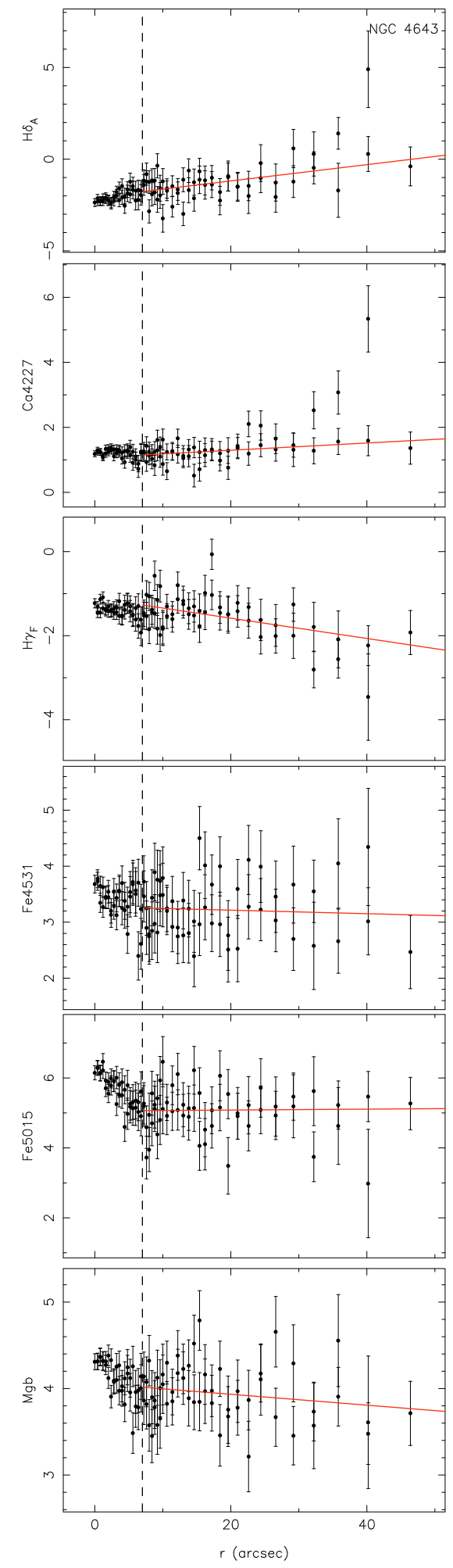

Fig. A.1. continued.
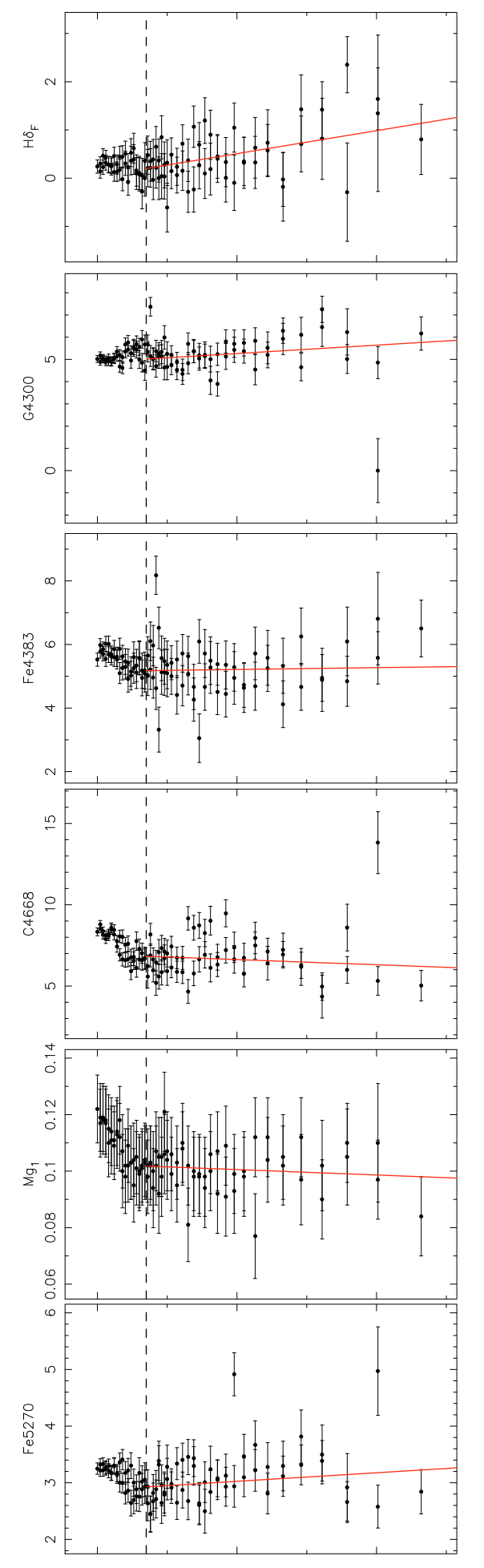
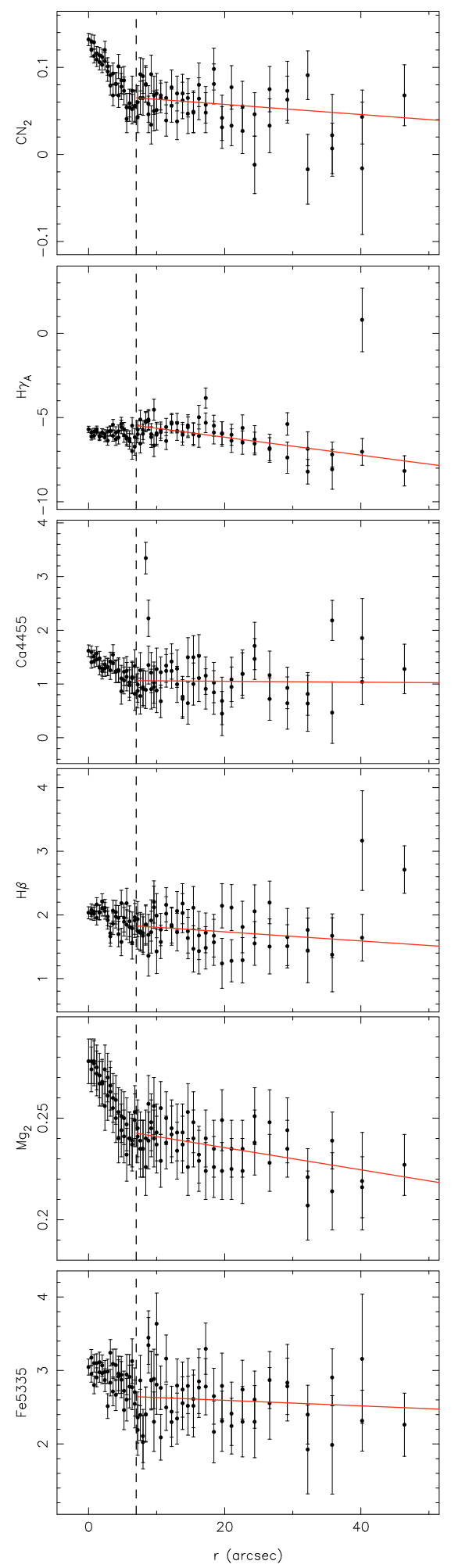
I. Pérez et al.: Study of the stellar line-strength indices and kinematics along bars. I., Online Material p 20

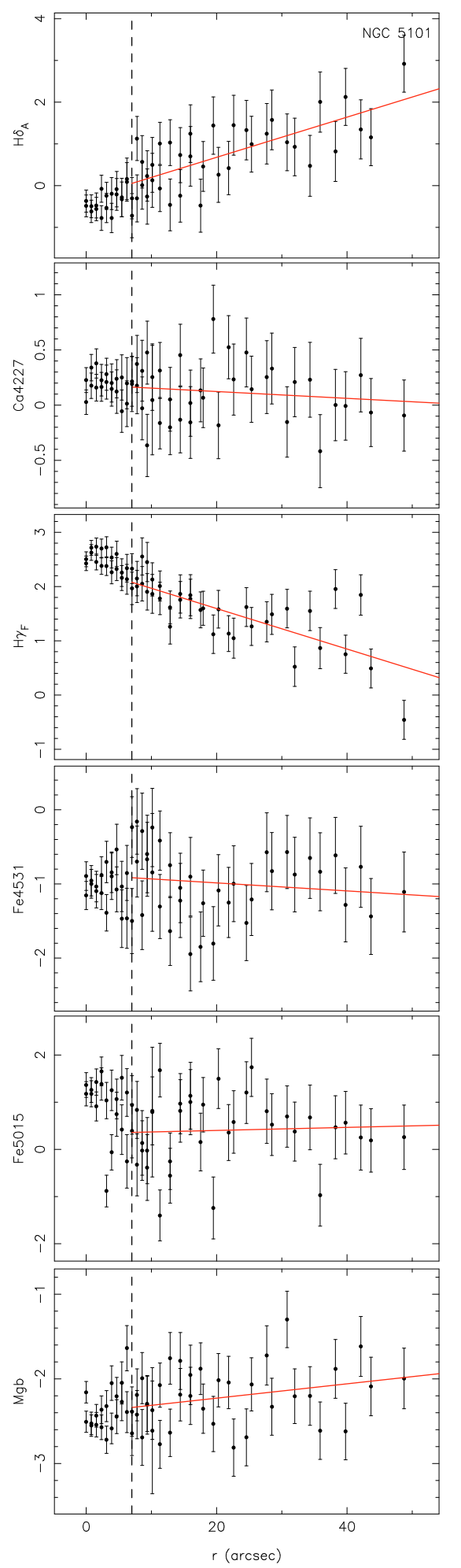

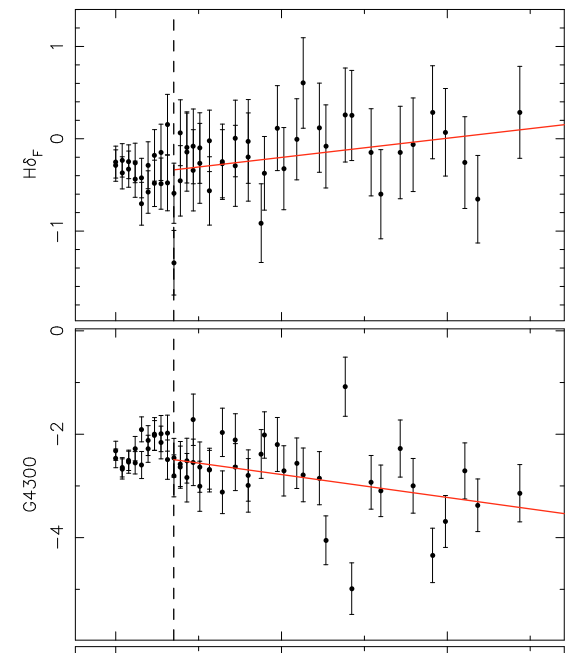
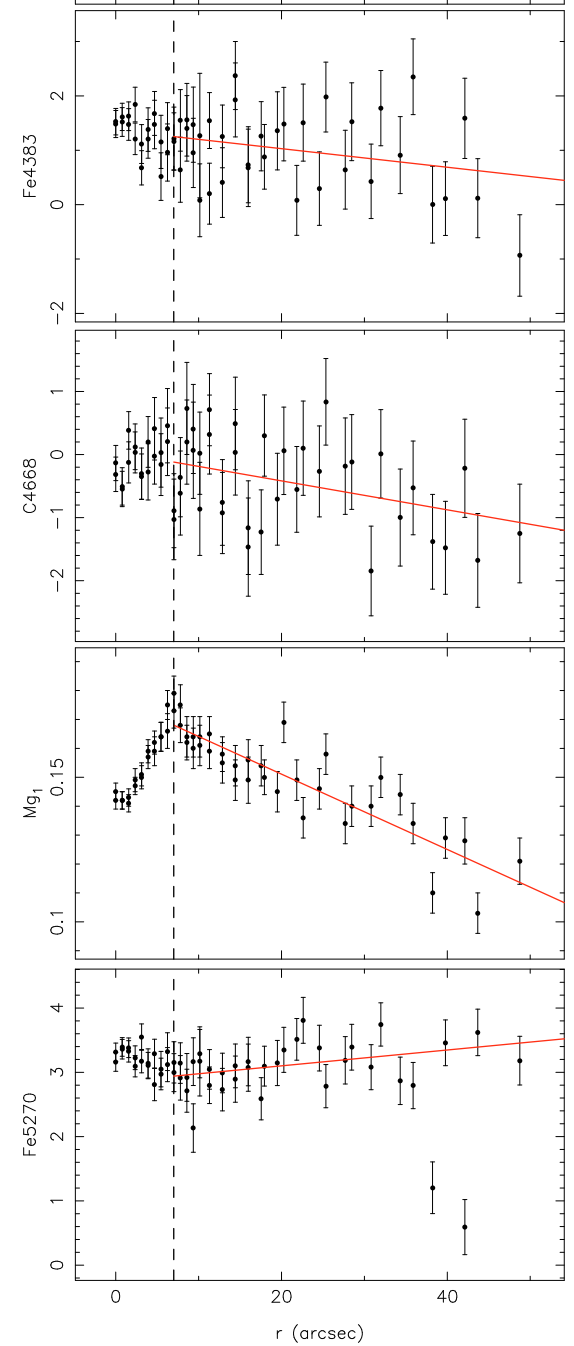
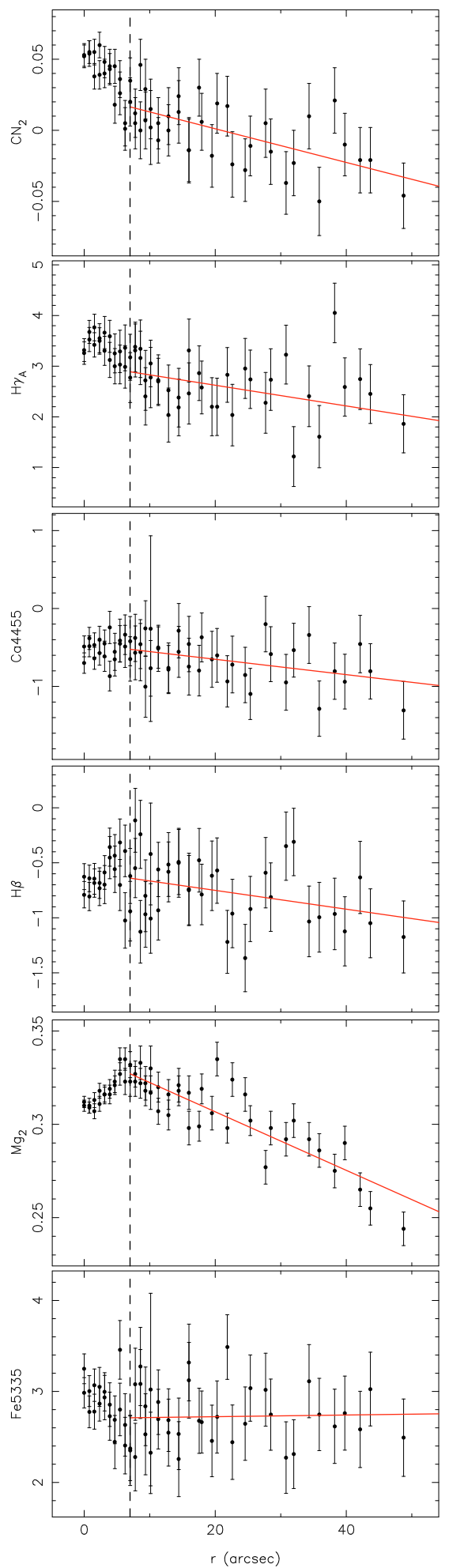

Fig. A.1. continued. 
I. Pérez et al.: Study of the stellar line-strength indices and kinematics along bars. I., Online Material p 21

\section{Appendix B: Calibration to the model spectrophotometric systems}

The original Lick/IDS spectra were not flux-calibrated by means of a flux-standard stars but normalised to a quartz-iodide tungsten lamp. The resulting continuum shape cannot be reproduced and causes small offsets for the indices with a broad wavelength range. Furthermore, the wavelength dependent resolution of the Lick spectrograph is also difficult to reproduce, which causes differences in other indices with larger dependence on resolution. In order to compare our measured indices with models based on this library we have to calculate these offsets. To calculate them, we observed 11 and 35 stars in common with the Lick/IDS library. By comparing the indices measured in our stars with those in the Lick/IDS database for the same objects, we derived mean offsets for all the indices observed. Figure B.1 shows this comparison. We did not find any systematic difference between the offset obtained with the stars of the first and second run and, therefore, we calculated just one offset for the two runs. The final offsets are listed in Table B.1
Table B.1. Mean offsets measured in the stars in common between our observing runs and MILES (first column) and Lick/IDS (second column).

\begin{tabular}{lrr}
\hline \hline Index & $\begin{array}{r}\text { Offset (MILES) } \\
\text { this work-miles }\end{array}$ & $\begin{array}{r}\text { Offset (Lick) } \\
\text { this work-Lick }\end{array}$ \\
\hline $\mathrm{H} \delta_{\mathrm{A}}$ & -0.18 & 0.00 \\
$\mathrm{H} \delta_{\mathrm{F}}$ & 0.000 & 0.15 \\
$\mathrm{CN}_{1}$ & 0.000 & -0.011 \\
$\mathrm{CN}_{2}$ & 0.000 & 0.00 \\
$\mathrm{Ca} 4227$ & 0.0 & 0.00 \\
$\mathrm{G} 4300$ & 0.2 & 0.0 \\
$\mathrm{H} \gamma_{\mathrm{A}}$ & 0.0 & 0.00 \\
$\mathrm{H} \gamma_{\mathrm{F}}$ & 0.0 & 0.00 \\
$\mathrm{~F} 4383$ & 0.21 & 0.00 \\
$\mathrm{Ca} 4455$ & 0.09 & -0.25 \\
$\mathrm{Fe} 4531$ & 0.12 & 0.00 \\
$\mathrm{C} 4668$ & -0.75 & -0.50 \\
$\mathrm{H} \beta$ & 0.00 & 0.163 \\
$\mathrm{Fe} 5015$ & 0.00 & 0.00 \\
$\mathrm{Mg} 1$ & -0.017 & -0.030 \\
$\mathrm{Mg} 2$ & 0.0 & -0.030 \\
$\mathrm{Mgb}$ & 0.0 & 0.00 \\
$\mathrm{Fe} 5270$ & 0.0 & 0.0 \\
$\mathrm{Fe} 5335$ & 0.0 & 0.0 \\
$\mathrm{Fe} 5406$ & 0.0 & 0.0 \\
\hline & &
\end{tabular}


I. Pérez et al.: Study of the stellar line-strength indices and kinematics along bars. I., Online Material p 22
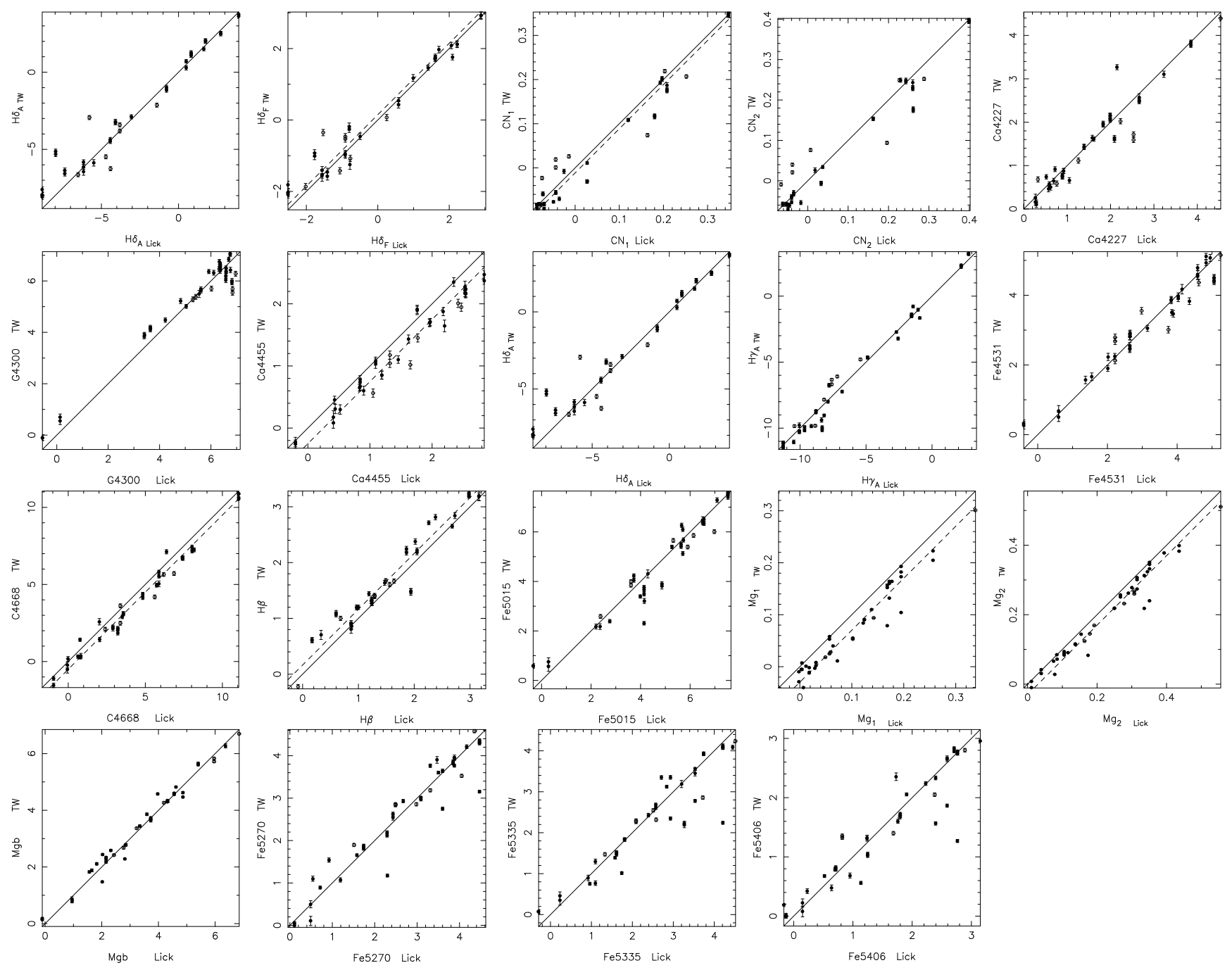

Fig. B.1. Comparison of the indices measured in the stars in common between this work and the Lick/IDS library. 
I. Pérez et al.: Study of the stellar line-strength indices and kinematics along bars. I., Online Material p 23

\section{Appendix C: Stellar population fits}

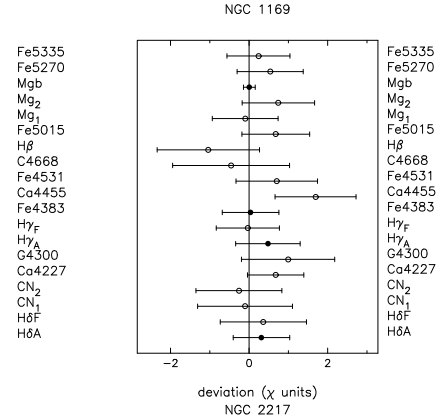

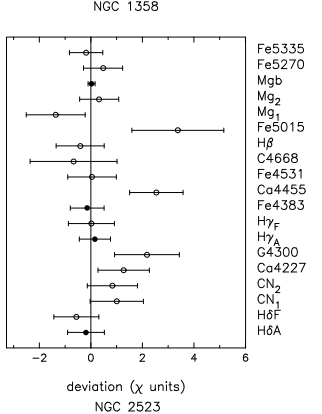

NGC 1433

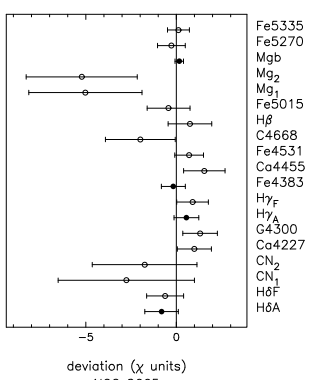

deviotion $(x$ units)
NGC 2665
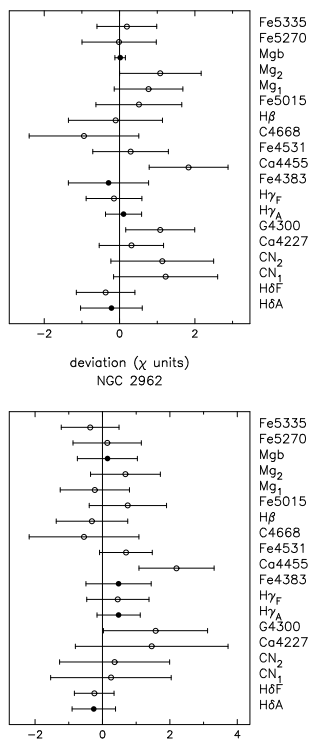

deviation $(x$ units)
NGC 4394
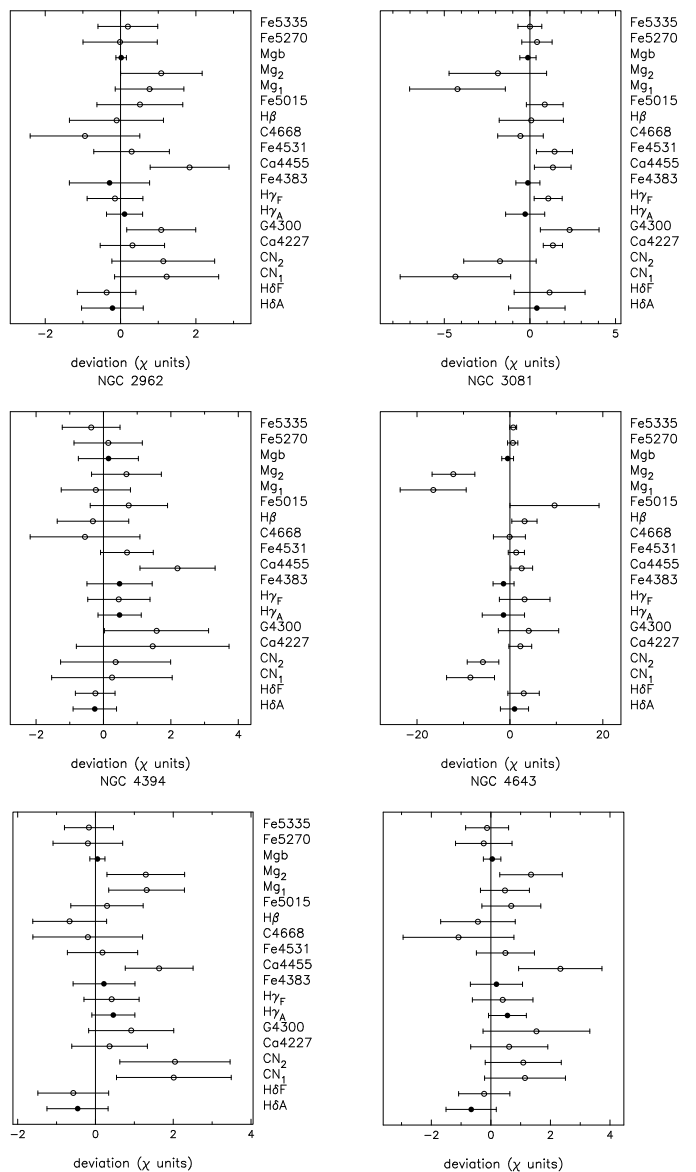

NGC 1832
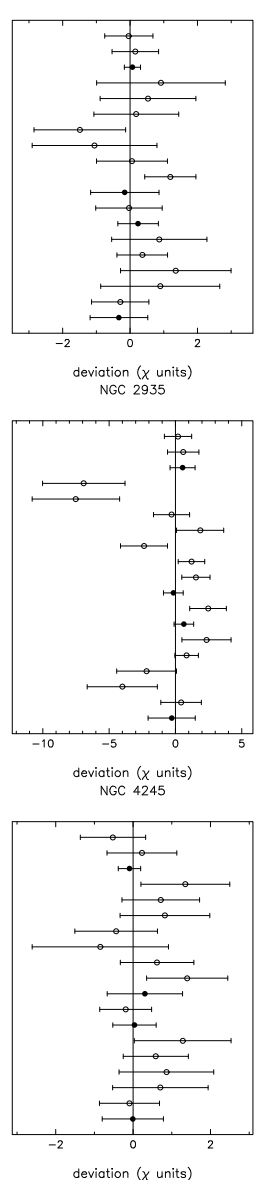

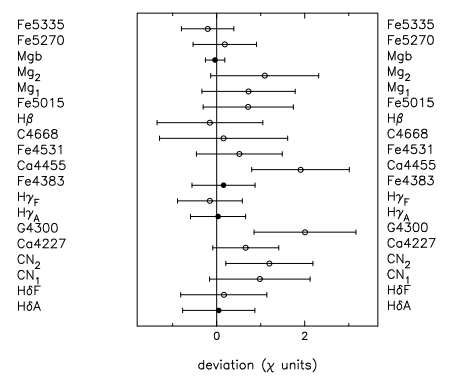

Fig. C.1. Deviations from best fit values for data compared to the models of TMB03. The indices that have been used for the fit are represented with filled symbols. 